\title{
Melicopteline A-E, Unusual Cyclopeptide Alkaloids with Antiviral Activity against Influenza A Virus from Melicope pteleifolia
}

Ba Wool Lee, ${ }^{\dagger}$ Thi Kim Quy Ha, ${ }^{\dagger}{ }^{\dagger}$ Eun Jin Park, ${ }^{\dagger}$ Hyo Moon Cho, ${ }^{\dagger}$ Byeol Ryu,${ }^{\dagger}$ Thi Phuong Doan, ${ }^{\dagger}$ Hee Ju Lee, ${ }^{\dagger, \S}$ and Won Keun $\mathrm{Oh}^{*}{ }^{* \dagger}$

${ }^{\dagger}$ Korea Bioactive Natural Material Bank, Research Institute of Pharmaceutical Sciences, College of Pharmacy, Seoul National University, Seoul 08826, Republic of Korea

Cantho University, Campus II, Cantho City 900000, Vietnam

${ }^{\S}$ Natural Product Informatics Research Center, Korea Institute of Science and Technology, Gangneung 25451, Republic of Korea 


\section{Contents:}

Figure S1: ${ }^{1} \mathrm{H}$ NMR spectrum of compound $1\left(800 \mathrm{MHz}, \mathrm{DMSO}-d_{6}\right)$.

Figure S2: ${ }^{13} \mathrm{C}$ NMR spectrum of compound $1\left(800 \mathrm{MHz}, \mathrm{DMSO}-d_{6}\right)$.

Figure S3: HSQC NMR spectrum of compound 1 (800 MHz, DMSO- $\left.d_{6}\right)$.

Figure S4: HMBC NMR spectrum of compound 1 ( $\left.800 \mathrm{MHz}, \mathrm{DMSO}-d_{6}\right)$.

Figure S5: COSY NMR spectrum of compound $1\left(800 \mathrm{MHz}, \mathrm{DMSO}-d_{6}\right)$.

Figure S6: TOCSY NMR spectrum of compound 1 ( $800 \mathrm{MHz}$, DMSO- $\left.d_{6}\right)$.

Figure S7: ROESY NMR spectrum of compound 1 (800 MHz, DMSO- $\left.d_{6}\right)$.

Figure S8: HR-ESIMS of compound 1.

Figure S9: MS/MS spectrum analysis of compound 1.

Figure S10: ECD spectrum of compound 1.

Figure S11: ${ }^{1} \mathrm{H}$ NMR spectrum of compound 2 ( $\left.800 \mathrm{MHz}, \mathrm{DMSO}-d_{6}\right)$.

Figure S12: ${ }^{13} \mathrm{C}$ NMR spectrum of compound $2\left(800 \mathrm{MHz}\right.$, DMSO- $\left.d_{6}\right)$.

Figure S13: HSQC NMR spectrum of compound 2 (800 MHz, DMSO- $\left.d_{6}\right)$.

Figure S14: HMBC NMR spectrum of compound 2 (800 MHz, DMSO- $\left.d_{6}\right)$.

Figure S15: COSY NMR spectrum of compound 2 (800 MHz, DMSO- $\left.d_{6}\right)$.

Figure S16: ROESY NMR spectrum of compound 2 ( $800 \mathrm{MHz}$, DMSO- $d_{6}$ ).

Figure S17: HR-ESIMS of compound 2.

Figure S18: MS/MS spectrum analysis of compound 2.

Table S1: Mass information of observed fragmented ion of compounds 1 and 2.

Figure S19: ECD spectrum of compound 2.

Figure S20: ${ }^{1} \mathrm{H}$ NMR spectrum of compound $3\left(800 \mathrm{MHz}, \mathrm{DMSO}-d_{6}\right)$.

Figure S21: ${ }^{13} \mathrm{C}$ NMR spectrum of compound $3\left(800 \mathrm{MHz}\right.$, DMSO- $\left.d_{6}\right)$.

Figure S22: HSQC NMR spectrum of compound 3 (800 MHz, DMSO- $\left.d_{6}\right)$.

Figure S23: HMBC NMR spectrum of compound 3 (800 MHz, DMSO- $d_{6}$ ).

Figure S24: COSY NMR spectrum of compound 3 (800 MHz, DMSO- $\left.d_{6}\right)$.

Figure S25: ROESY NMR spectrum of compound 3 (800 MHz, DMSO- $\left.d_{6}\right)$. 
Figure S26: HR-ESIMS of compound 3.

Figure S27: ECD spectrum of compound $\mathbf{3}$.

Figure S28: ${ }^{1} \mathrm{H}$ NMR spectrum of compound $4\left(800 \mathrm{MHz}, \mathrm{DMSO}-d_{6}\right)$.

Figure S29: ${ }^{13} \mathrm{C}$ NMR spectrum of compound $4\left(800 \mathrm{MHz}, \mathrm{DMSO}-d_{6}\right)$.

Figure S30: HSQC NMR spectrum of compound $4\left(800 \mathrm{MHz}, \mathrm{DMSO}-d_{6}\right)$.

Figure S31: HMBC NMR spectrum of compound 4 (800 MHz, DMSO- $\left.d_{6}\right)$.

Figure S32: COSY NMR spectrum of compound 4 (800 MHz, DMSO- $\left.d_{6}\right)$.

Figure S33: ROESY NMR spectrum of compound 4 (800 MHz, DMSO- $\left.d_{6}\right)$.

Figure S34: HR-ESIMS of compound 4.

Figure S35: ECD spectrum of compound 4.

Figure S36: ${ }^{1} \mathrm{H}$ NMR spectrum of compound $5\left(800 \mathrm{MHz}\right.$, DMSO- $\left.d_{6}\right)$.

Figure S37: ${ }^{13} \mathrm{C}$ NMR spectrum of compound $5\left(800 \mathrm{MHz}\right.$, DMSO- $\left.d_{6}\right)$.

Figure S38: HSQC NMR spectrum of compound 5 (800 MHz, DMSO- $\left.d_{6}\right)$.

Figure S39: HMBC NMR spectrum of compound 5 (800 MHz, DMSO- $\left.d_{6}\right)$.

Figure S40: COSY NMR spectrum of compound 5 (800 MHz, DMSO- $\left.d_{6}\right)$.

Figure S41: ROESY NMR spectrum of compound 5 (800 MHz, DMSO- $\left.d_{6}\right)$.

Figure S42: HR-ESIMS of compound 5 .

Figure S43: ECD spectrum of compound 5.

Table S2: Comparison of retention times of derivatized amino acids in $\mathbf{1 - 5}$ to those of authentic amino acid.

Figure S44: Advanced Marfey's analysis of compound 1 for common amino acids.

Figure S45: Advanced Marfey's analysis of compound 1 for Thr.

Figure S46: Advanced Marfey's analysis of compound 2 for common amino acids.

Figure S47: Advanced Marfey's analysis of compounds 2 and 5 for Ile.

Figure S48: Advanced Marfey's analysis of compounds $\mathbf{3}$ and $\mathbf{4}$ for common amino acids.

Figure S49: Advanced Marfey's analysis of compounds 3 and $\mathbf{4}$ for Thr.

Figure S50: Advanced Marfey's analysis of compound $\mathbf{5}$ for common amino acids. 
Figure S51: Four possible configurations of HPI moiety.

Figure S52: Distance between $\mathrm{CH}_{3}-\gamma(\mathrm{Val})$ and $\mathrm{H}-2$ in 3D structures of four possible configurations of HPI.

Figure S53: Expanded ROESY spectrum for correlations between $\mathrm{CH}_{3}-\gamma($ Val) and $\mathrm{H}-2$.

Figure S54: Distance between $\mathrm{CH}_{3}-\gamma(\mathrm{Val})$ and protons at the junction of HPI in 3D structures of four possible configurations of HPI.

Figure S55: Expanded ROESY spectrum for correlations between $\mathrm{CH}_{3}-\gamma$ (Val) and protons at the junction of HPI.

Figure S56: Expanded ROESY spectrum for correlations between H-8a and H-2 of HPI.

Table S3: Comparison of chemical shifts of $\mathrm{H}-2$ and $\mathrm{H}-8$ a to values in a reference.

Figure S57: Structures of two diastereomers of HPI.

Figure S58: Expanded ROESY spectrum for correlations between $\mathrm{CH}_{3}-\gamma($ Val) and aromatic protons of HPI.

Figure S59: Chromatograms of EtOAc fraction of $M$. pteleifolia and extracted ion chromatograms of compounds $\mathbf{3}$ and $\mathbf{5}$ containing Trp instead of HPI. 


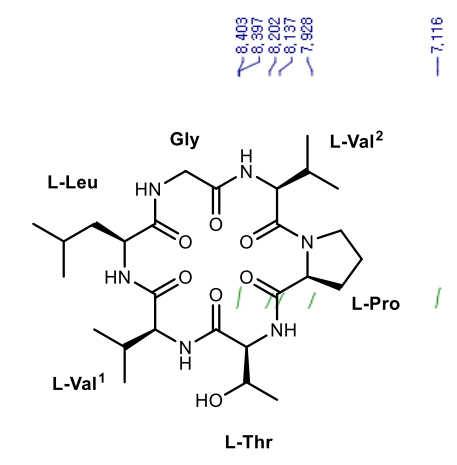

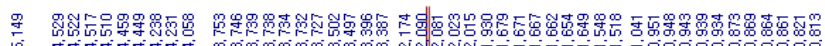

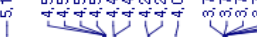

1

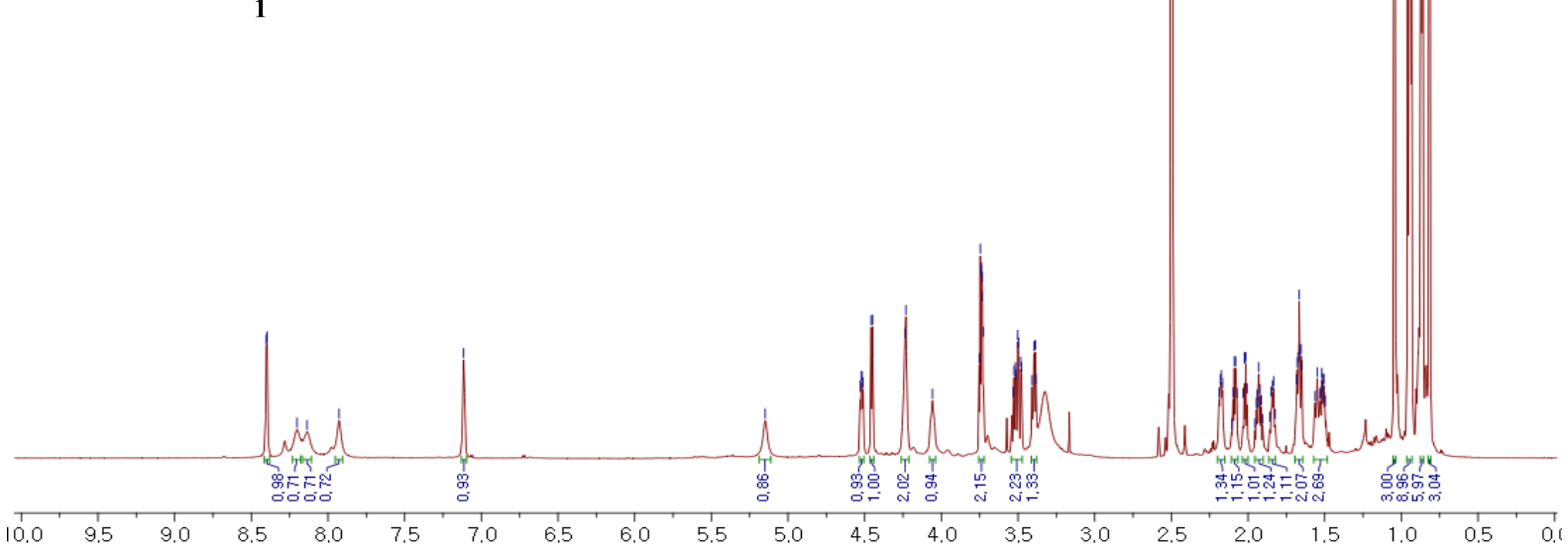

Figure S1: ${ }^{1} \mathrm{H}$ NMR spectrum of compound $1\left(800 \mathrm{MHz}, \mathrm{DMSO}-d_{6}\right)$.

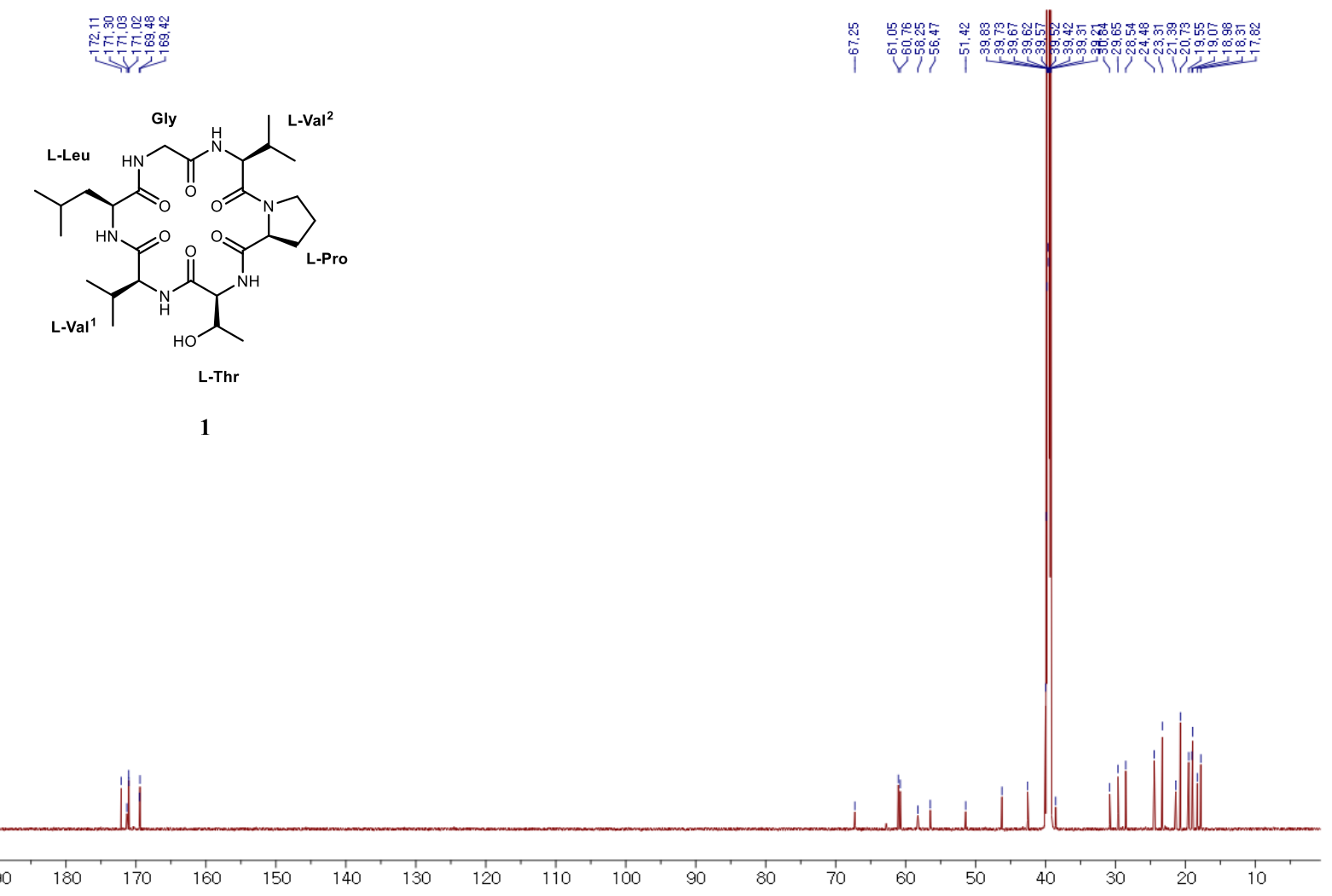

Figure S2: ${ }^{13} \mathrm{C}$ NMR spectrum of compound $1\left(800 \mathrm{MHz}, \mathrm{DMSO}-d_{6}\right)$. 


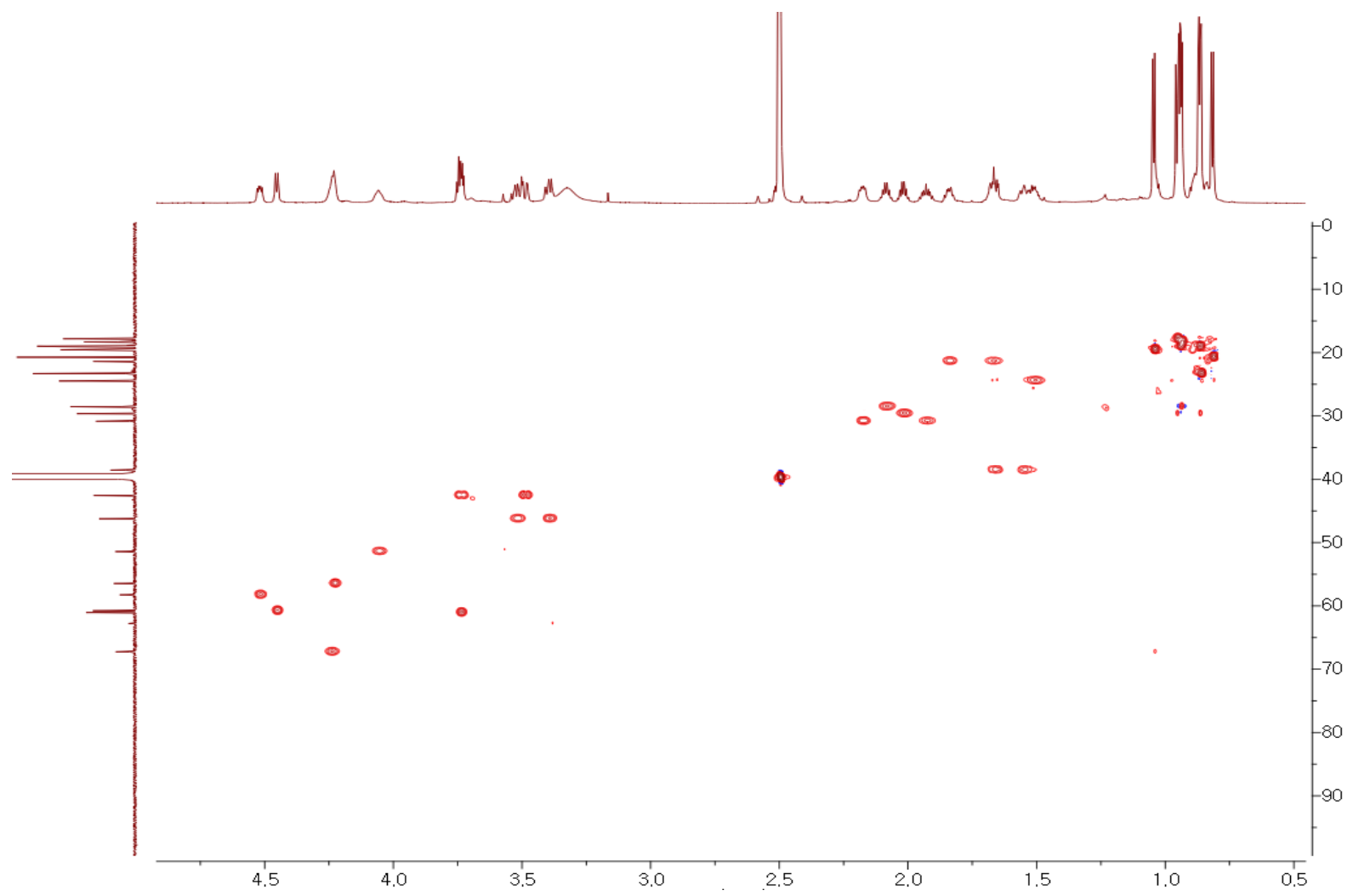

Figure S3: HSQC NMR spectrum of compound $1\left(800 \mathrm{MHz}, \mathrm{DMSO}-d_{6}\right)$.

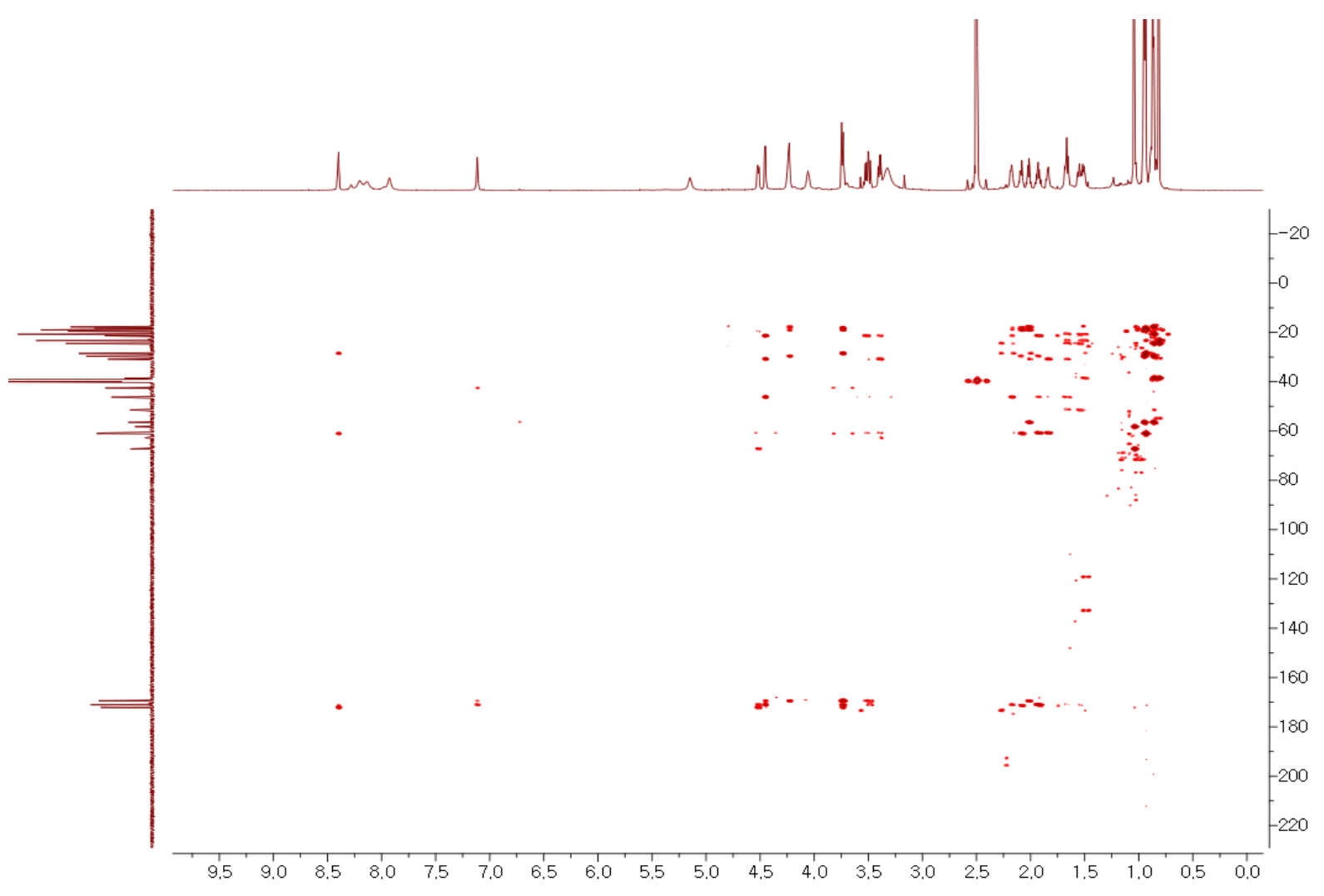

Figure S4: HMBC NMR spectrum of compound $1\left(800 \mathrm{MHz}, \mathrm{DMSO}-d_{6}\right)$. 


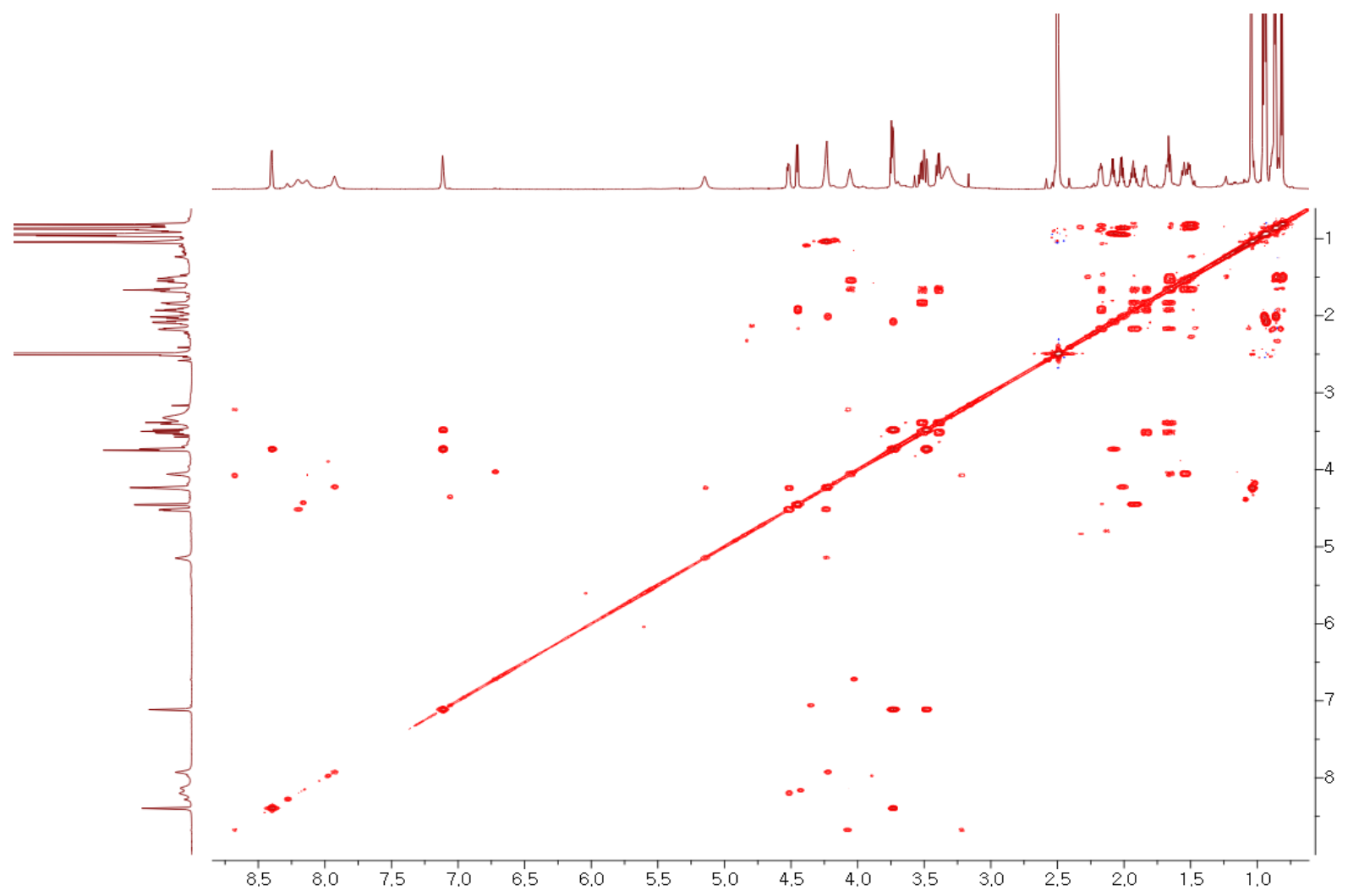

Figure S5: COSY NMR spectrum of compound $1\left(800 \mathrm{MHz}, \mathrm{DMSO}-d_{6}\right)$.

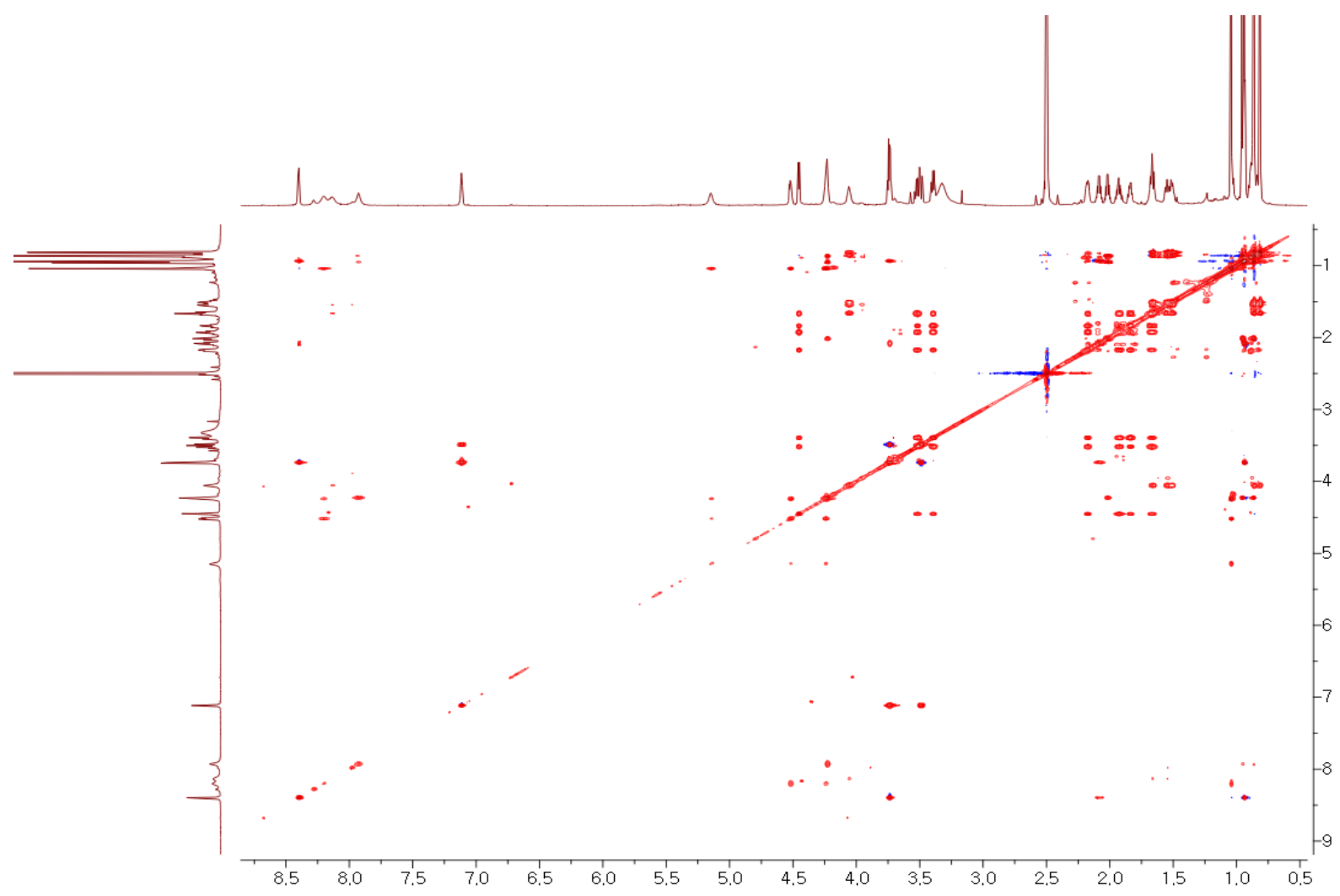

Figure S6: TOCSY NMR spectrum of compound $1\left(800 \mathrm{MHz}, \mathrm{DMSO}-d_{6}\right)$. 


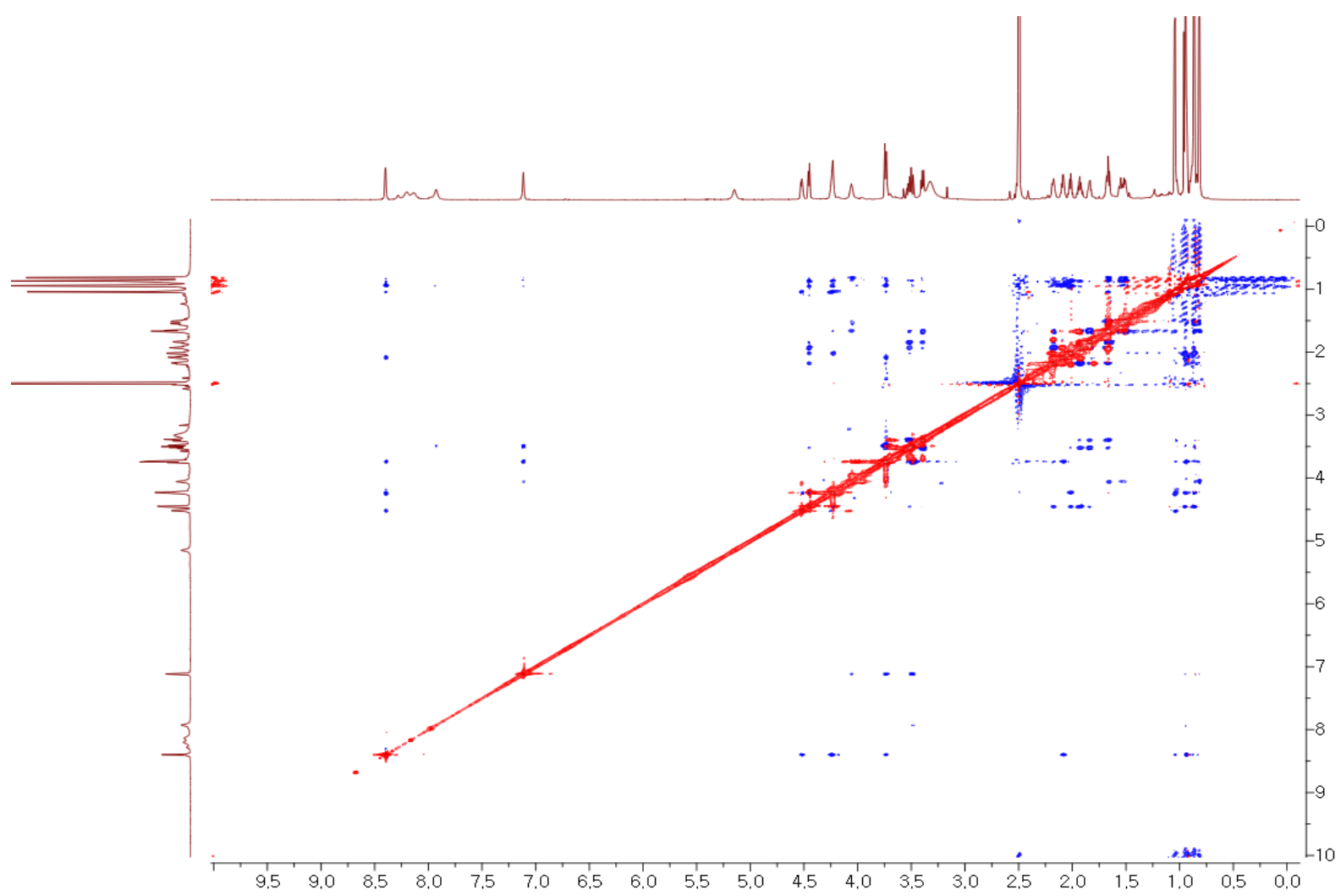

Figure S7: ROESY NMR spectrum of compound 1 (800 MHz, DMSO- $\left.d_{6}\right)$.

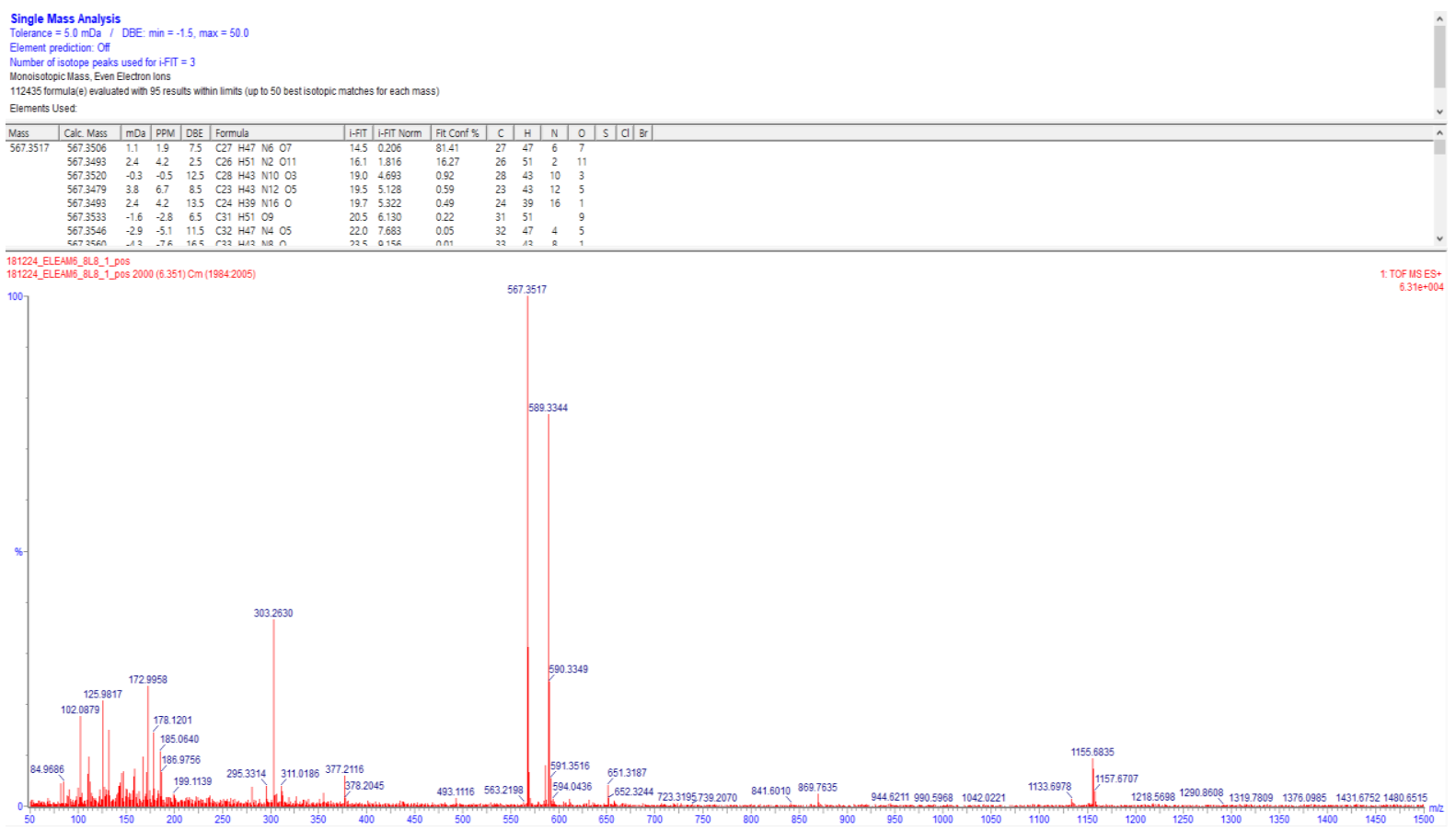

Figure S8: HR-ESIMS of compound 1. 


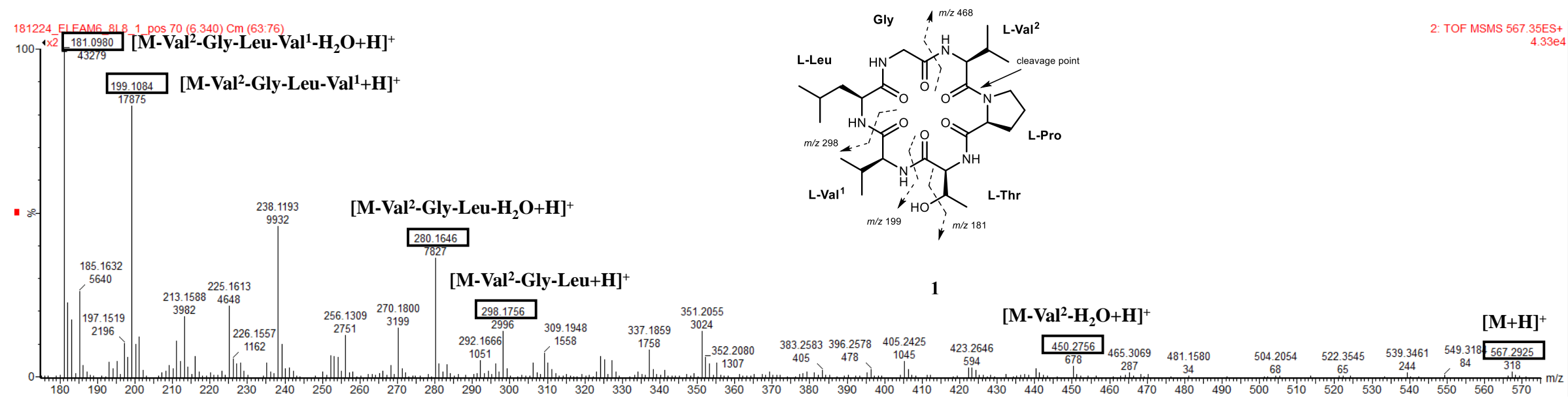

Figure S9: MS/MS spectrum analysis of compound 1 


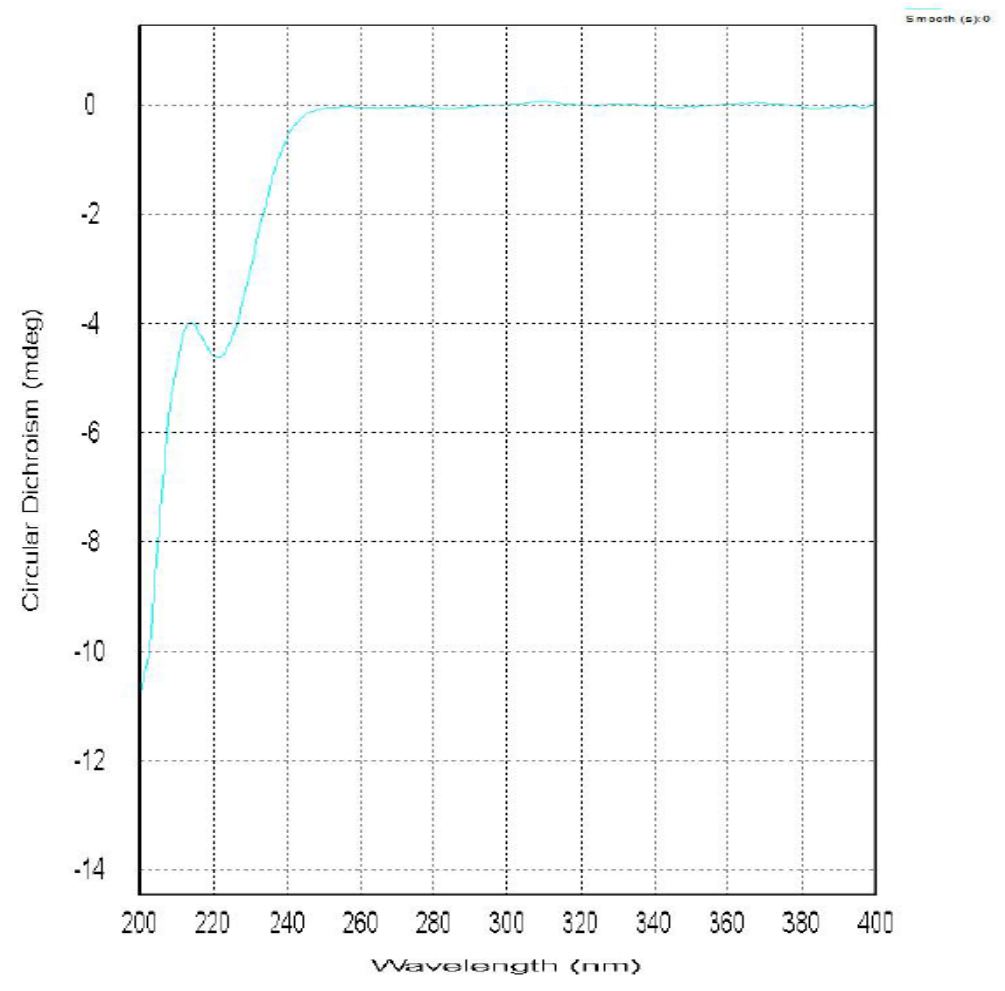

Figure S10: ECD spectrum of compound 1.

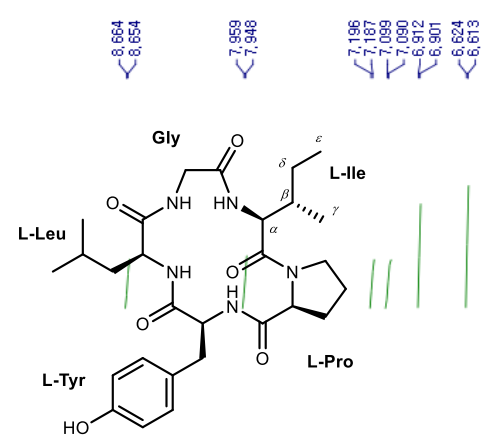

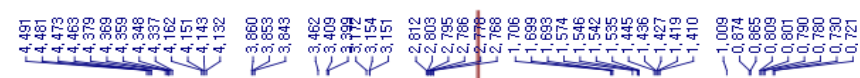

2

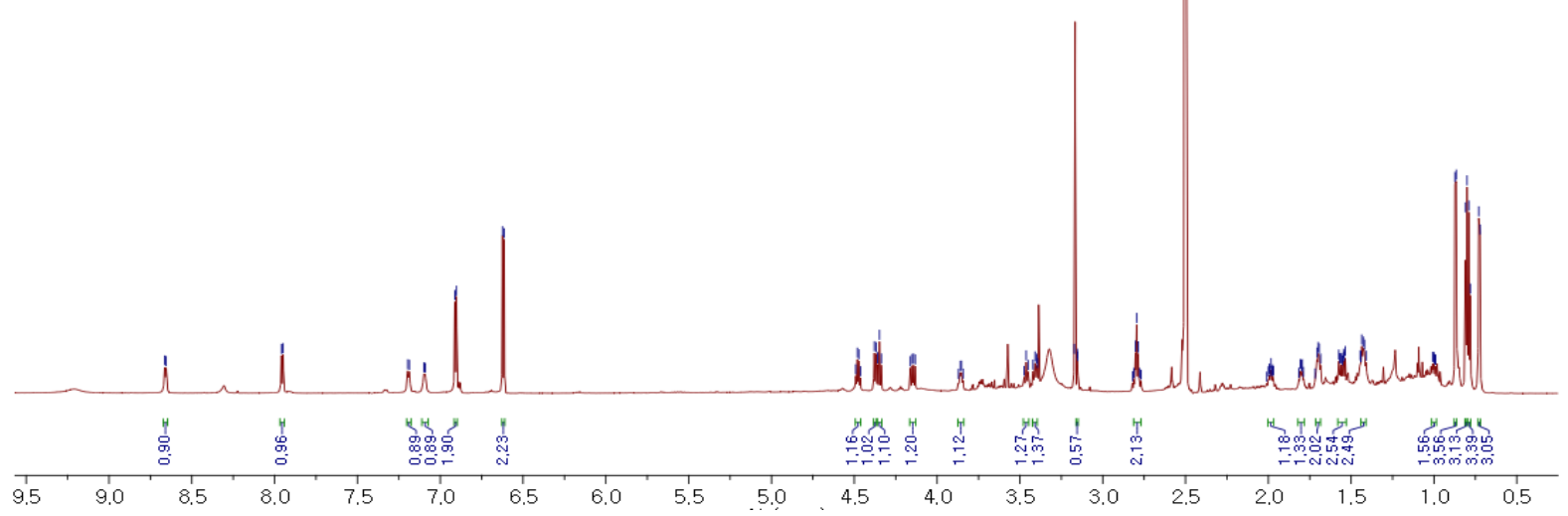

Figure S11: ${ }^{1} \mathrm{H}$ NMR spectrum of compound $2\left(800 \mathrm{MHz}, \mathrm{DMSO}-d_{6}\right)$. 


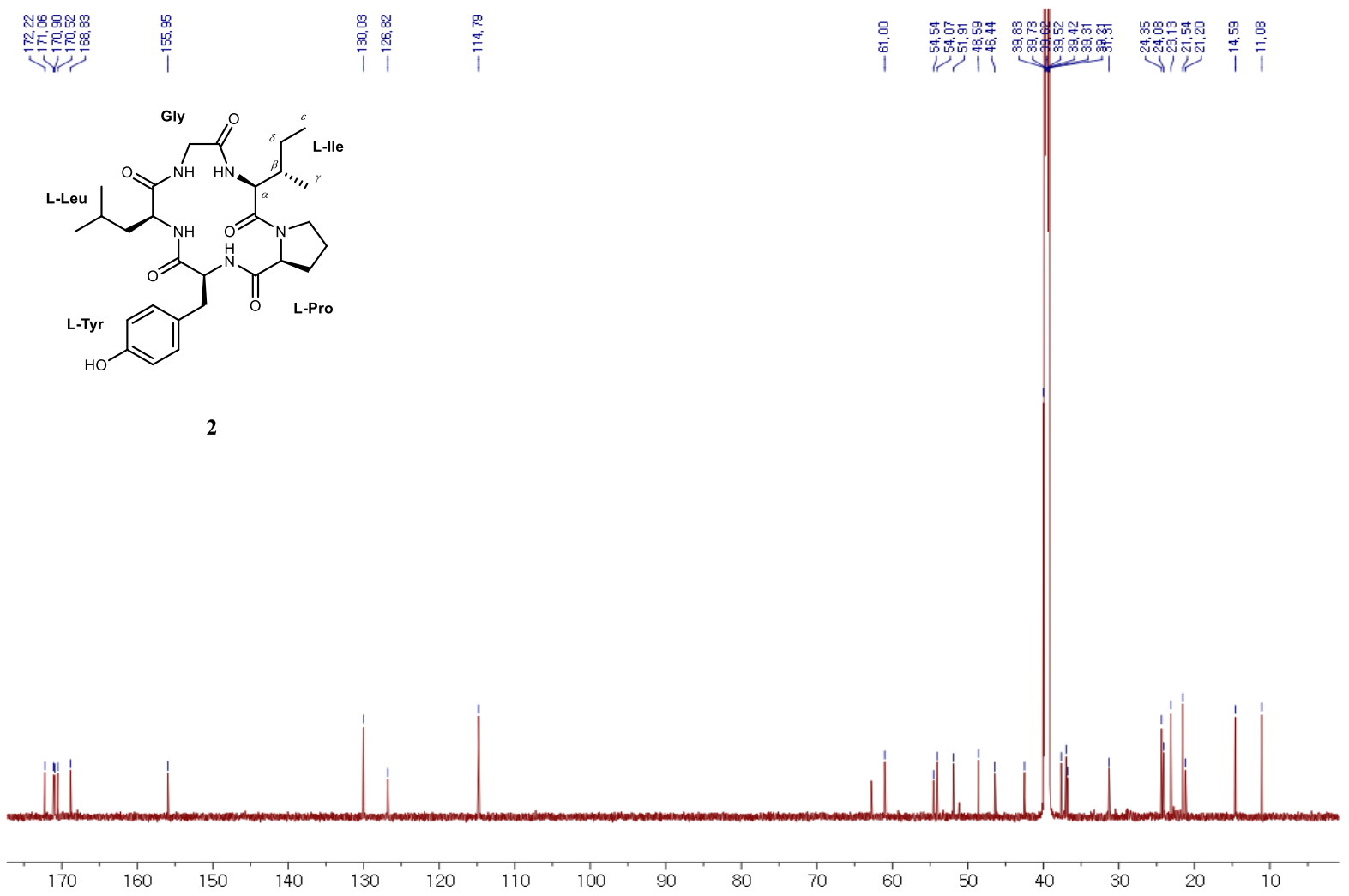

Figure S12: ${ }^{13} \mathrm{C}$ NMR spectrum of compound $2\left(800 \mathrm{MHz}, \mathrm{DMSO}-d_{6}\right)$.

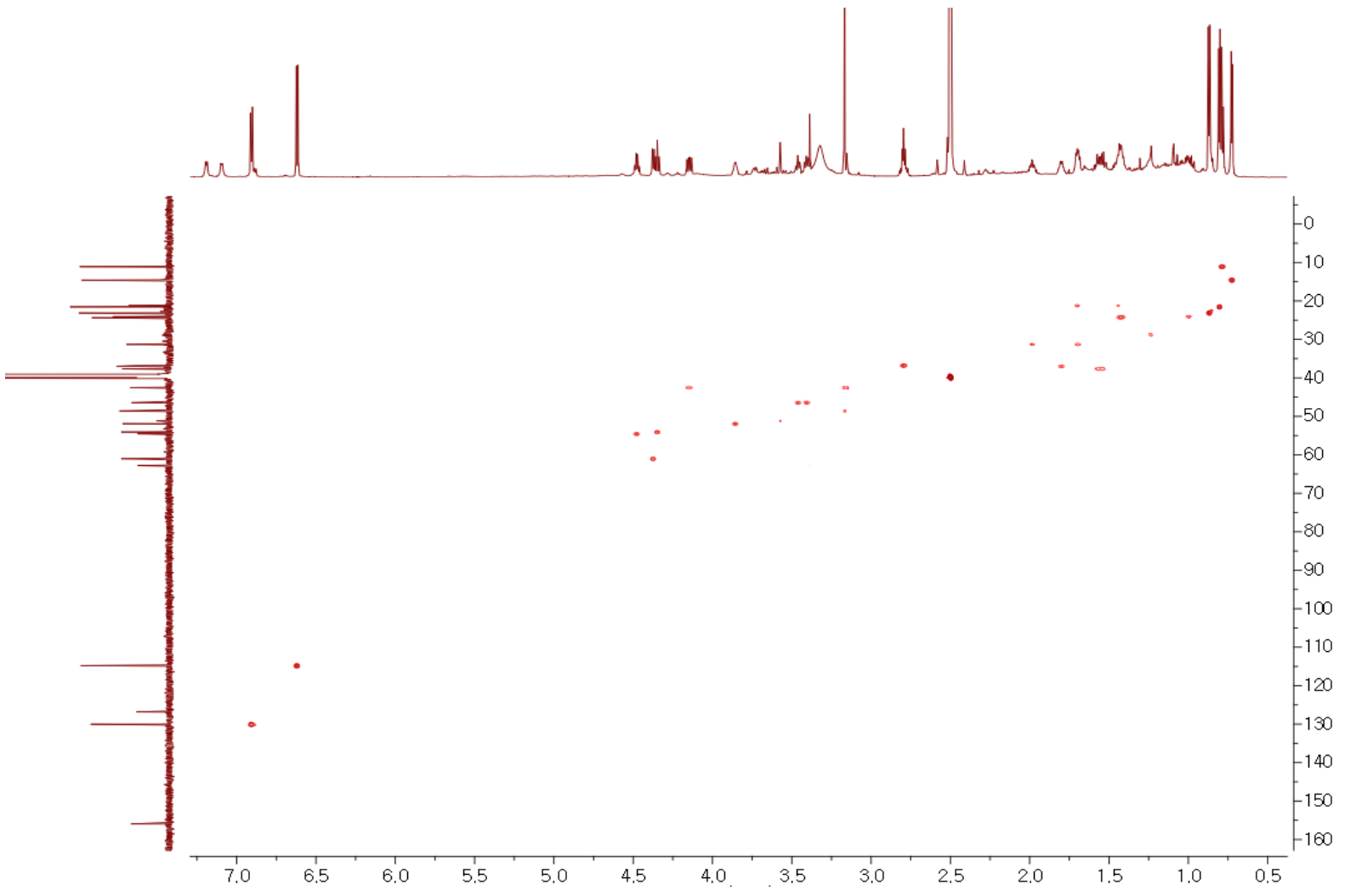

Figure S13: HSQC NMR spectrum of compound 2 ( $800 \mathrm{MHz}$, DMSO- $d_{6}$ ). 


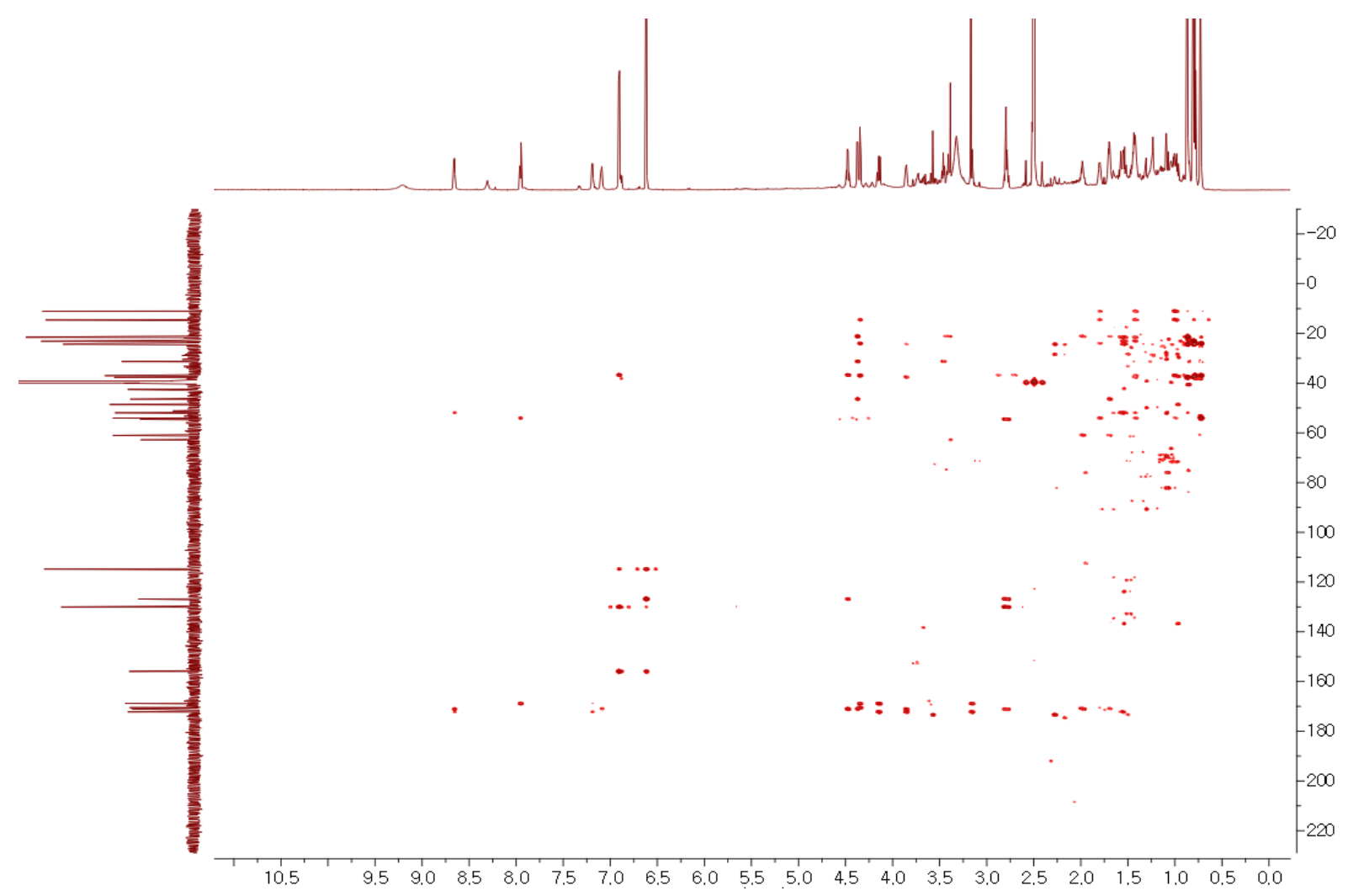

Figure S14: HMBC NMR spectrum of compound 2 (800 MHz, DMSO- $\left.d_{6}\right)$.

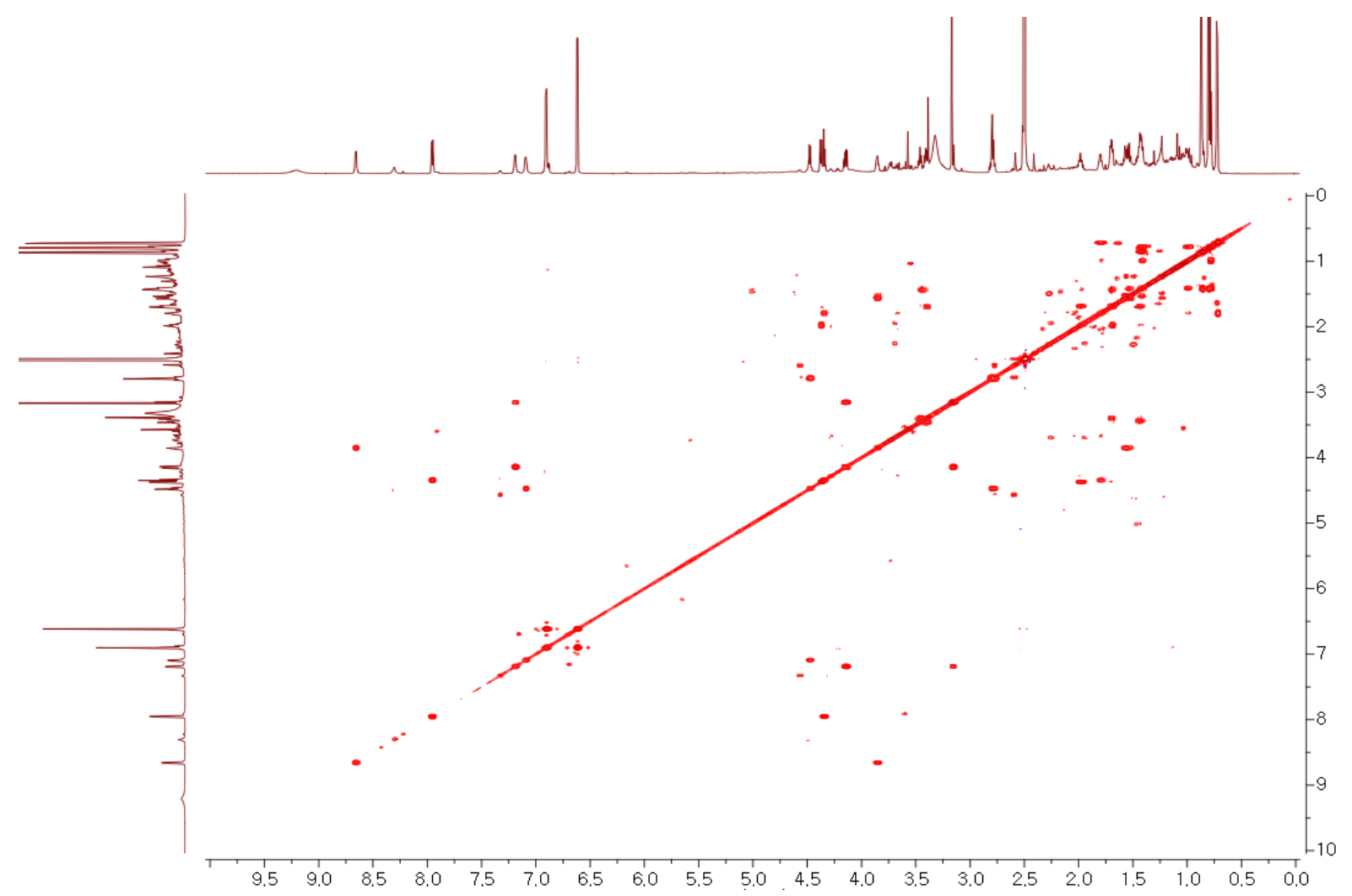

Figure S15: COSY NMR spectrum of compound 2 ( $\left.800 \mathrm{MHz}, \mathrm{DMSO}-d_{6}\right)$. 


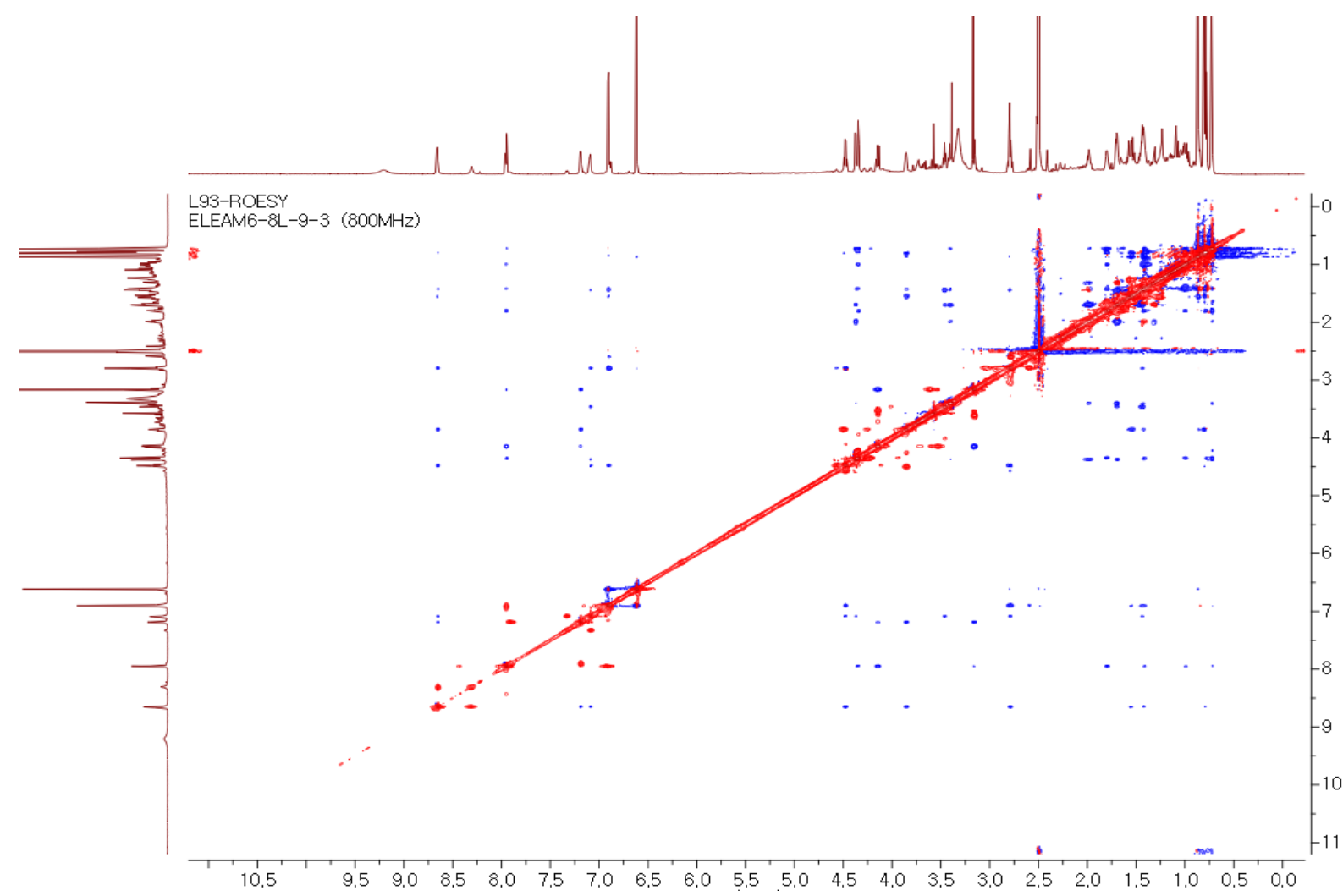

Figure S16: ROESY NMR spectrum of compound $2\left(800 \mathrm{MHz}, \mathrm{DMSO}-d_{6}\right)$.

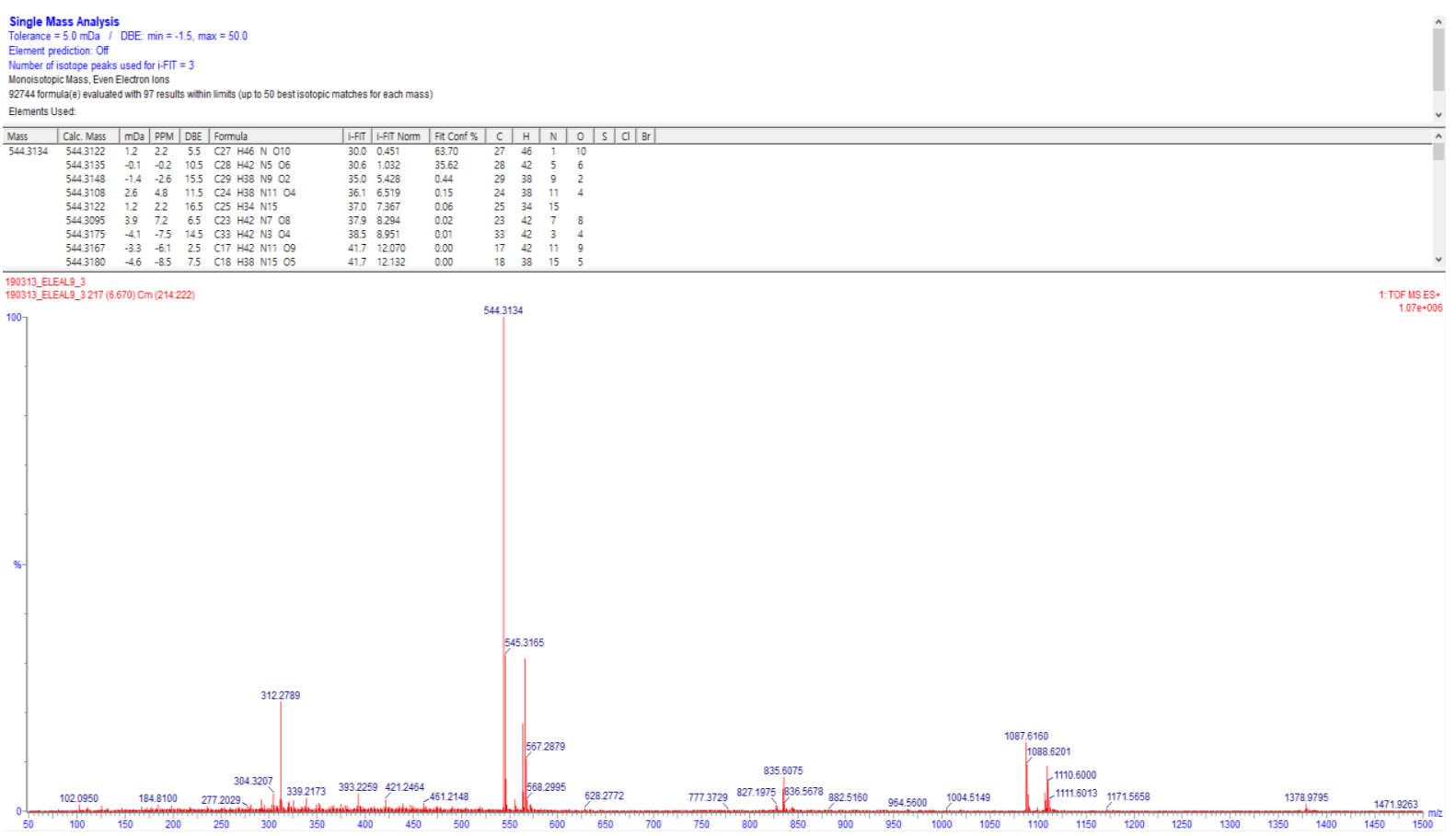

Figure S17: HR-ESIMS of compound 2 


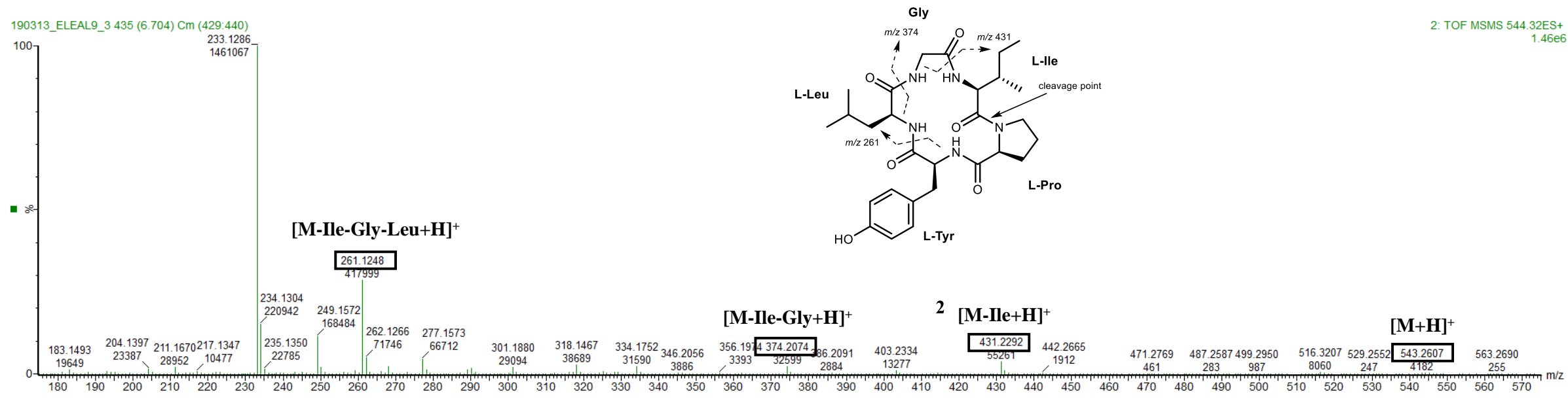

Figure S18: MS/MS spectrum analysis of compound 2. 
Table S1: Mass information of observed fragmented ion of compounds $\mathbf{1}$ and 2.

\begin{tabular}{llllll}
\hline & Accurate (observed) & Calculated MS & $\begin{array}{l}\text { Difference } \\
\text { (ppm) }\end{array}$ & Formula & Fragmented ion of a sequence \\
\hline cpd 1 & 468.2838 & 468.2822 & 3.4 & $\mathrm{C}_{22} \mathrm{H}_{38} \mathrm{~N}_{5} \mathrm{O}_{6}$ & $\mathrm{M}-\mathrm{Val}^{2}+\mathrm{H}$ \\
& 450.2756 & 450.2716 & 8.9 & $\mathrm{C}_{22} \mathrm{H}_{36} \mathrm{~N}_{5} \mathrm{O}_{5}$ & $\mathrm{M}-\mathrm{Val}^{2}-\mathrm{H}_{2} \mathrm{O}+\mathrm{H}$ \\
& 298.1756 & 298.1767 & -3.7 & $\mathrm{C}_{14} \mathrm{H}_{24} \mathrm{~N}_{3} \mathrm{O}_{4}$ & $\mathrm{M}-\mathrm{Val}^{2}-\mathrm{Gly}-\mathrm{Leu}+\mathrm{H}$ \\
& 280.1646 & 280.1661 & -5.4 & $\mathrm{C}_{14} \mathrm{H}_{22} \mathrm{~N}_{3} \mathrm{O}_{3}$ & $\mathrm{M}-\mathrm{Val}^{2}-\mathrm{Gly}-\mathrm{Leu}-\mathrm{H}_{2} \mathrm{O}+\mathrm{H}$ \\
& 199.1084 & 199.1083 & 0.5 & $\mathrm{C}_{9} \mathrm{H}_{15} \mathrm{~N}_{2} \mathrm{O}_{3}$ & $\mathrm{M}-\mathrm{Val}^{2}-\mathrm{Gly}-\mathrm{Leu}-\mathrm{Va}{ }^{1}+\mathrm{H}$ \\
cpd 2 & 181.0984 & 181.0977 & 1.7 & $\mathrm{C}_{9} \mathrm{H}_{13} \mathrm{~N}_{2} \mathrm{O}_{2}$ & $\mathrm{M}-\mathrm{Val}^{2}-\mathrm{Gly}-\mathrm{Leu}-\mathrm{Val}^{1}-\mathrm{H}_{2} \mathrm{O}+\mathrm{H}$ \\
& 431.2292 & 431.2294 & -0.5 & $\mathrm{C}_{22} \mathrm{H}_{31} \mathrm{~N}_{4} \mathrm{O}_{5}$ & $\mathrm{M}-\mathrm{Ile}^{\mathrm{H}} \mathrm{H}$ \\
& 374.2074 & 374.2080 & -1.6 & $\mathrm{C}_{20} \mathrm{H}_{28} \mathrm{~N}_{3} \mathrm{O}_{4}$ & $\mathrm{M}-\mathrm{Ile}-\mathrm{Gly}+\mathrm{H}$ \\
& 261.1248 & 261.1239 & 3.4 & $\mathrm{C}_{14} \mathrm{H}_{17} \mathrm{~N}_{2} \mathrm{O}_{3}$ & $\mathrm{M}-\mathrm{Ile}-\mathrm{Gly}-\mathrm{Leu}+\mathrm{H}$ \\
\hline
\end{tabular}




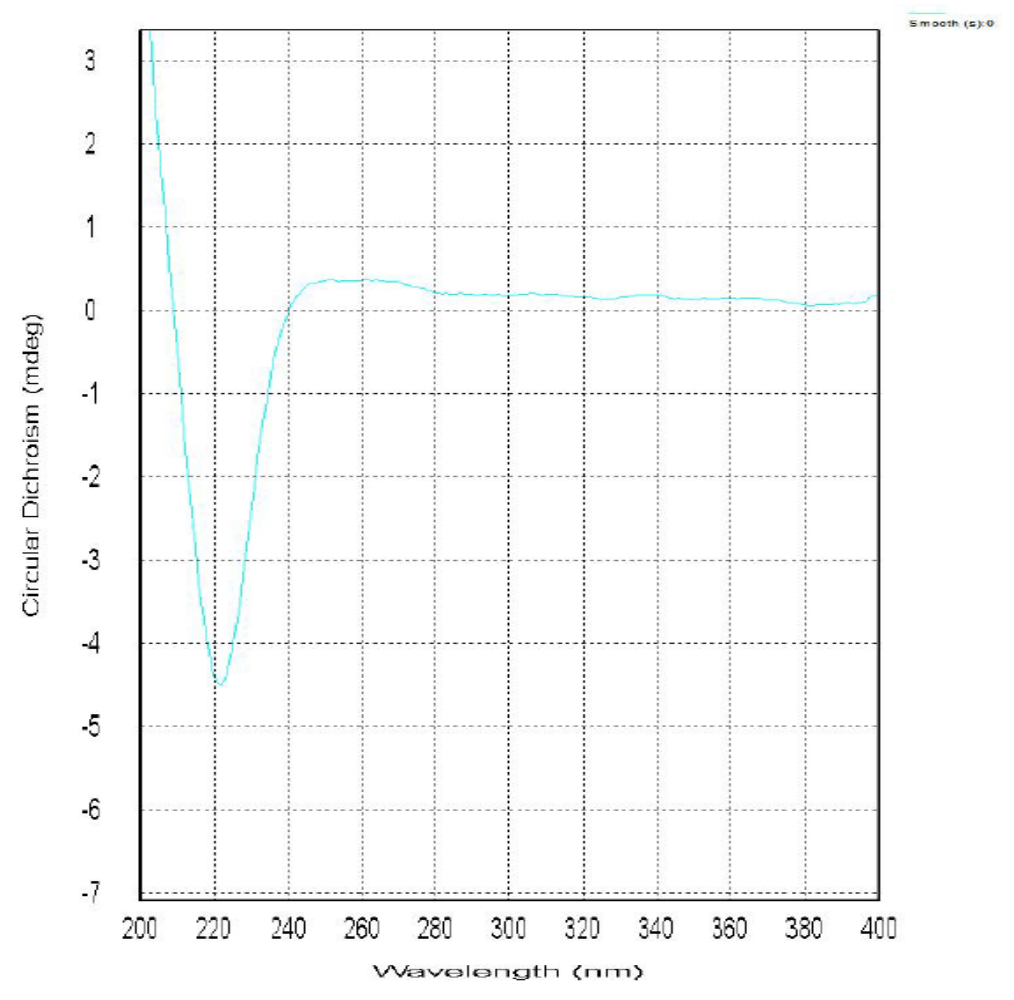

Figure S19: ECD spectrum of compound 2.

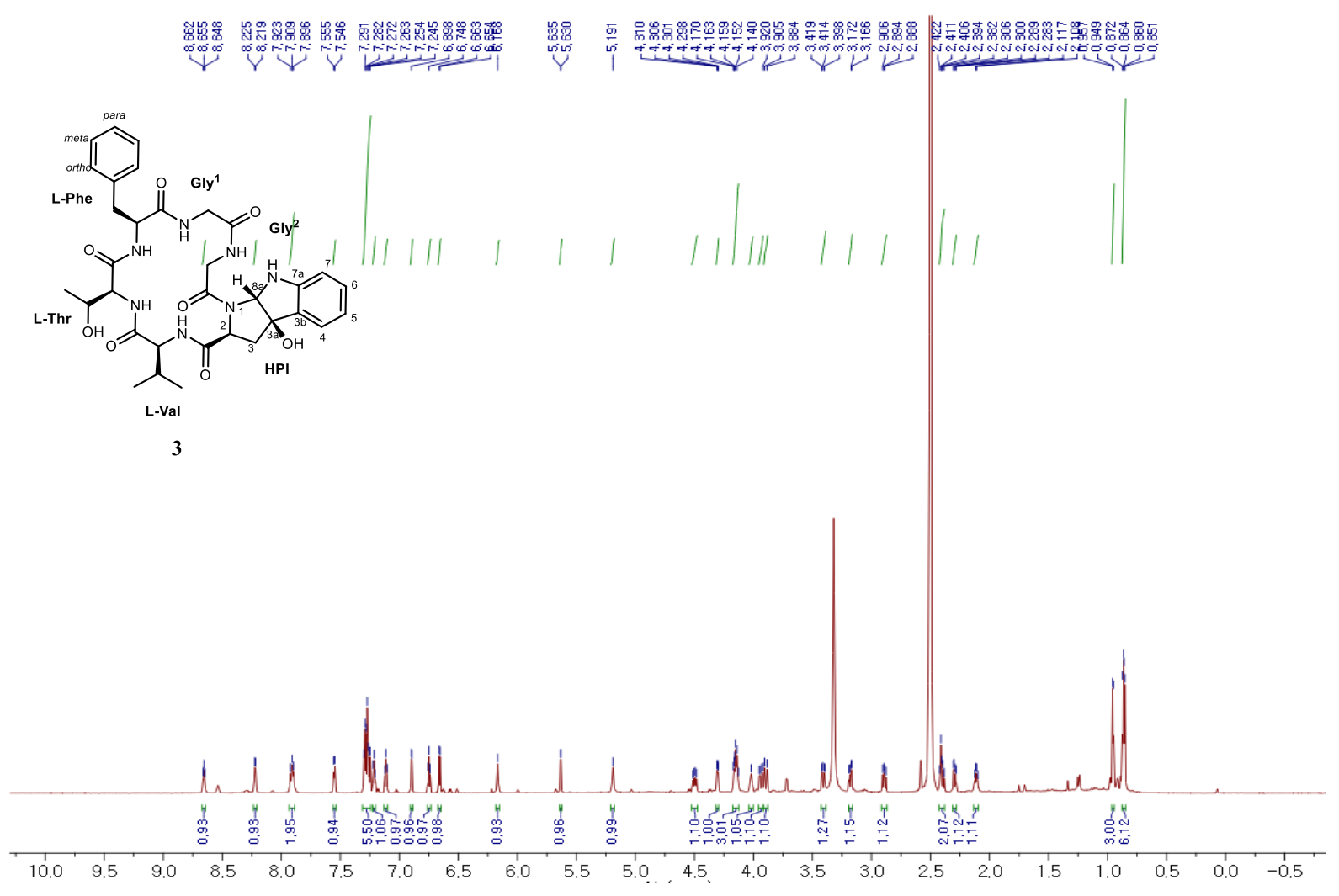

Figure S20: ${ }^{1} \mathrm{H}$ NMR spectrum of compound $3\left(800 \mathrm{MHz}, \mathrm{DMSO}-d_{6}\right)$. 


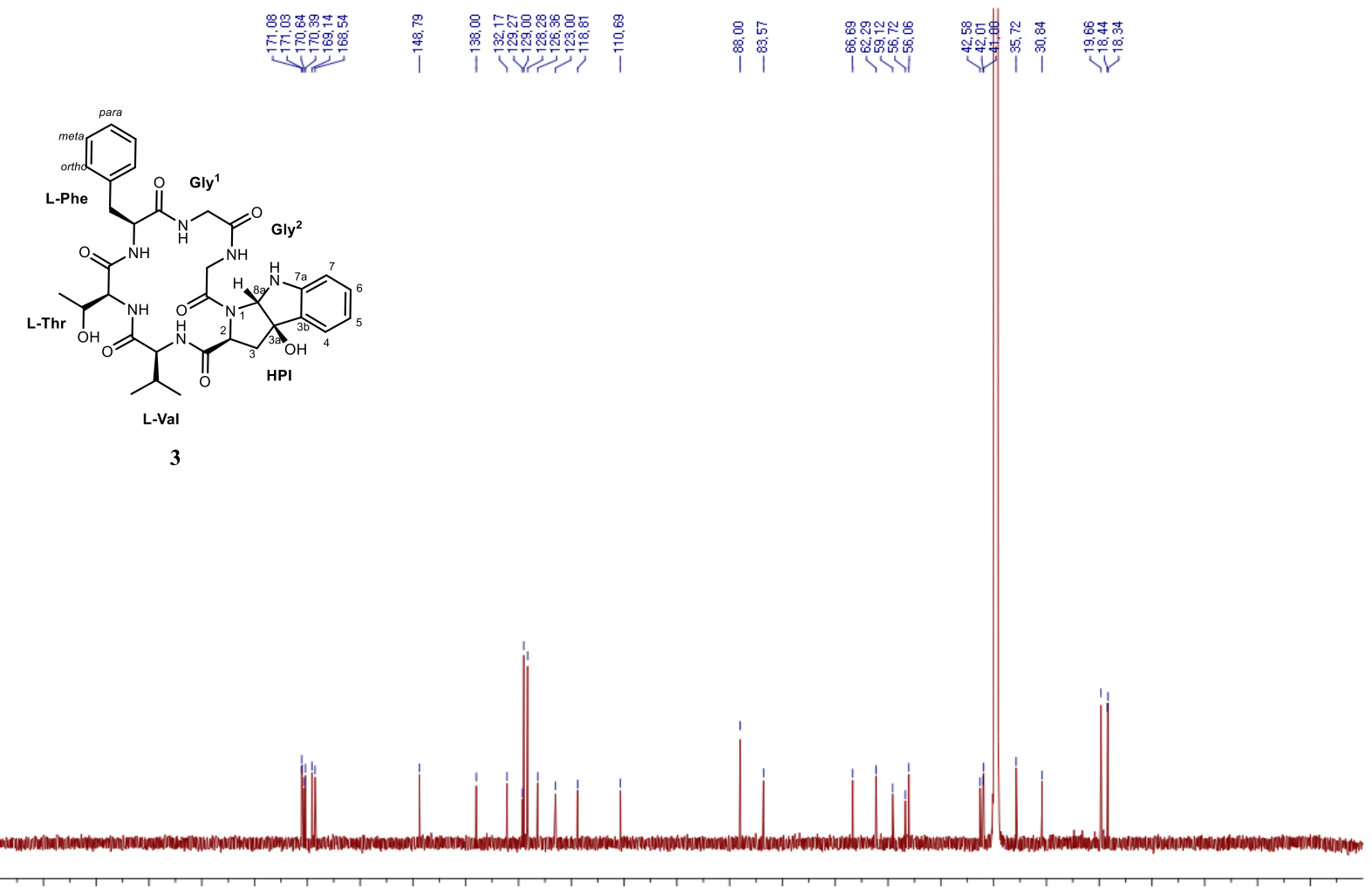

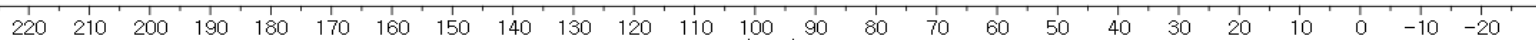

Figure S21: ${ }^{13} \mathrm{C}$ NMR spectrum of compound $3\left(800 \mathrm{MHz}\right.$, DMSO- $\left.d_{6}\right)$.

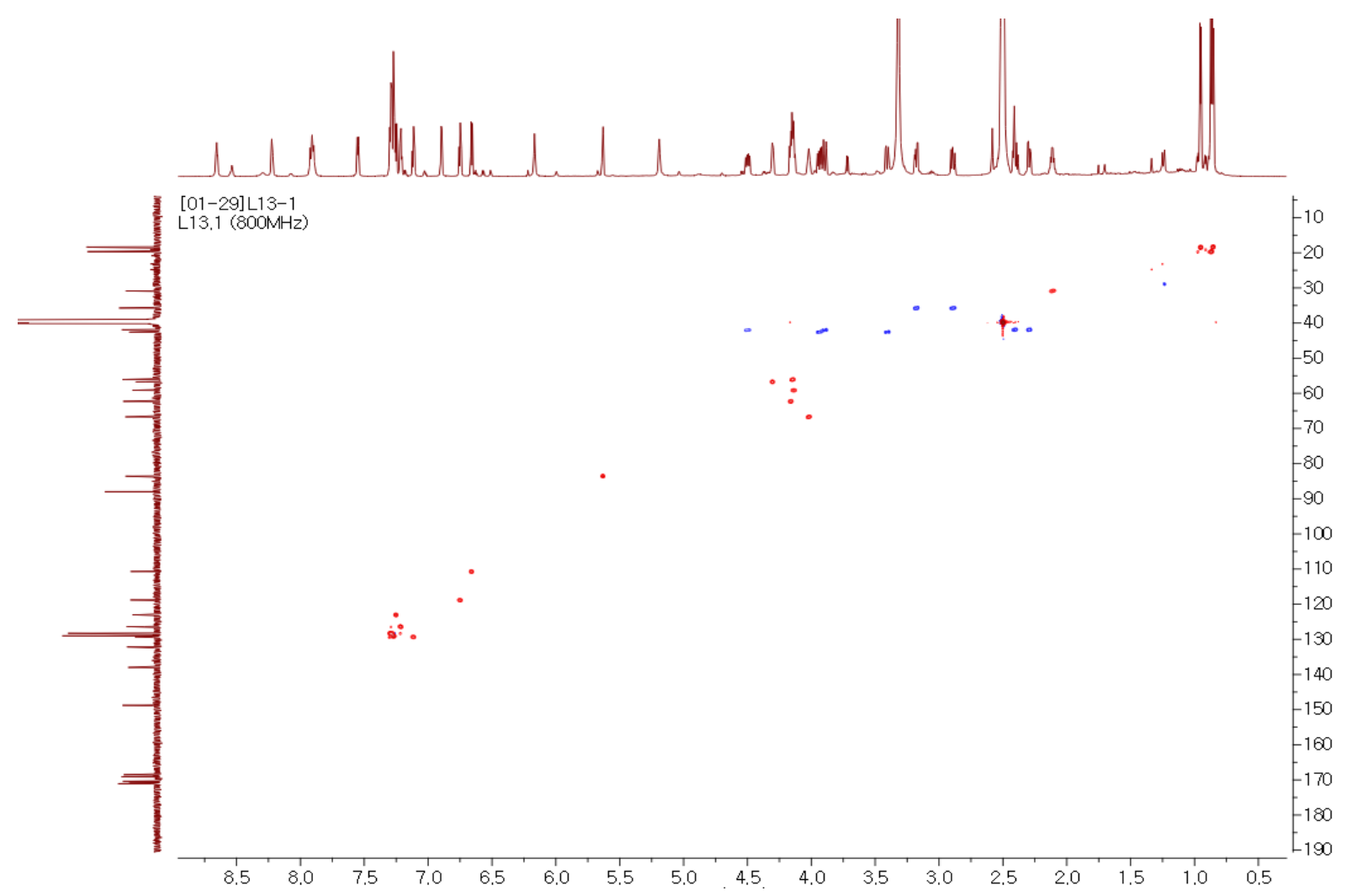

Figure S22: HSQC NMR spectrum of compound 3 ( $800 \mathrm{MHz}$, DMSO- $\left.d_{6}\right)$. 


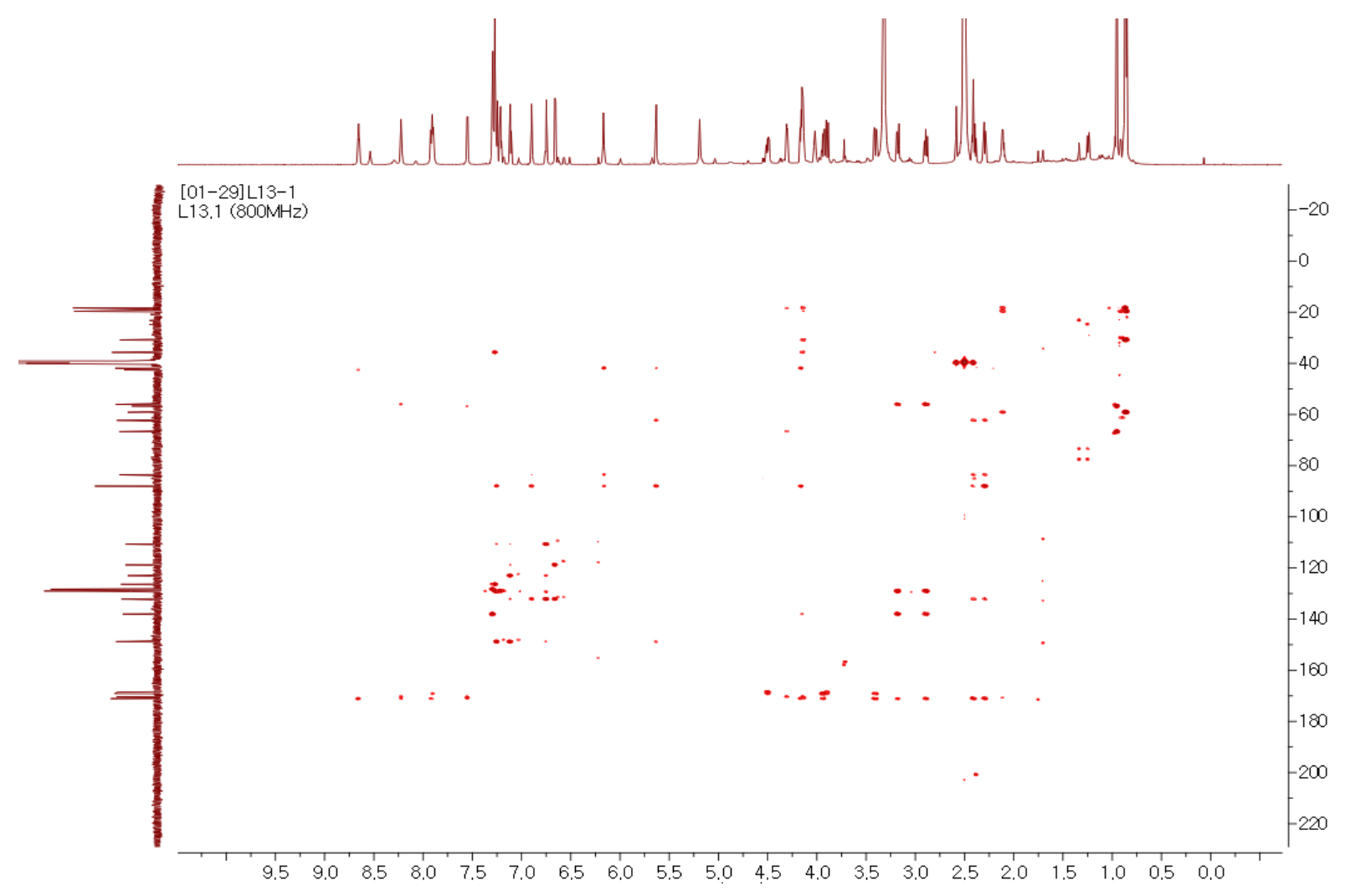

Figure S23: HMBC NMR spectrum of compound 3 (800 MHz, DMSO- $d_{6}$ ).

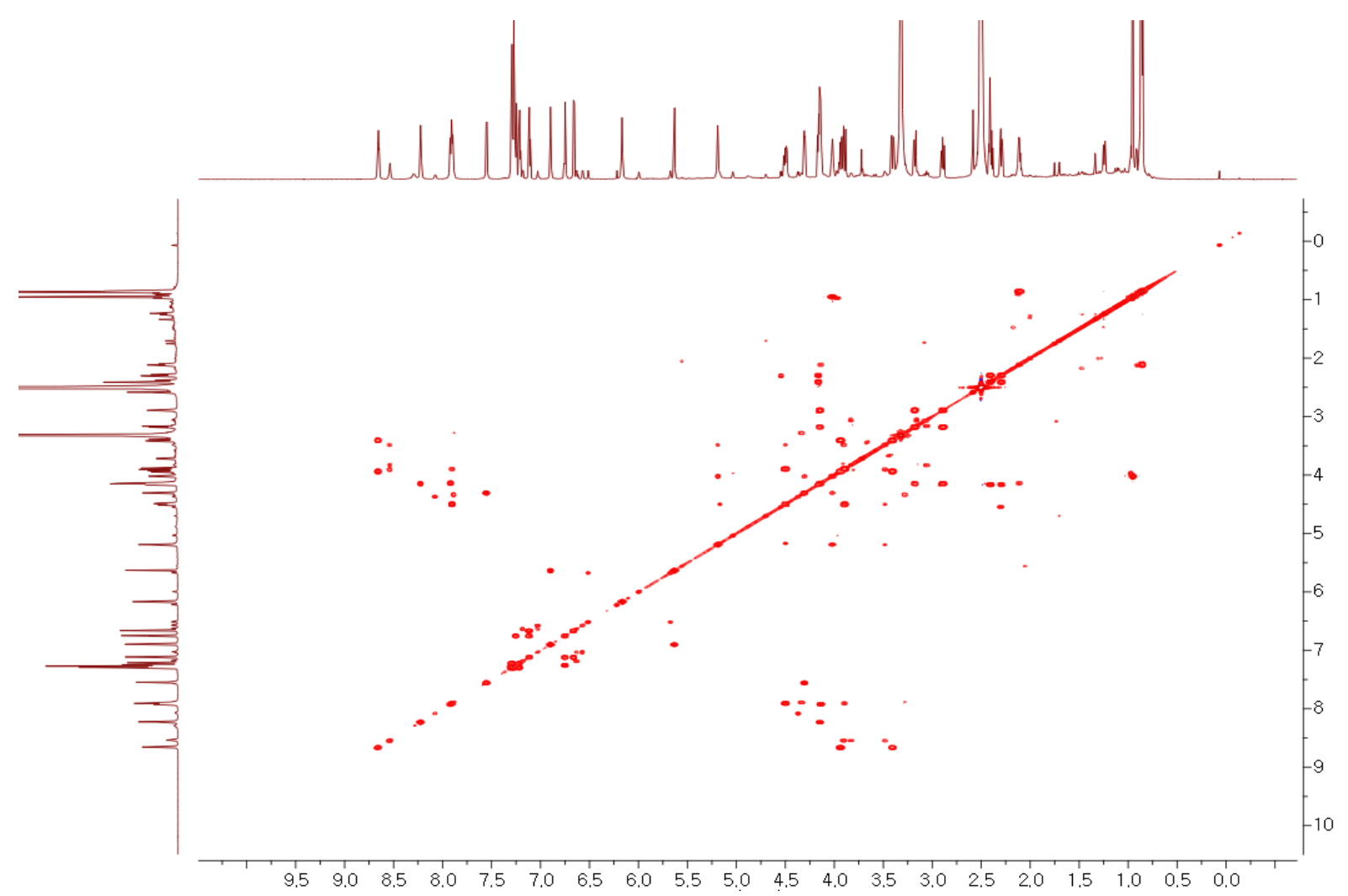

Figure S24: COSY NMR spectrum of compound 3 (800 MHz, DMSO- $\left.d_{6}\right)$. 


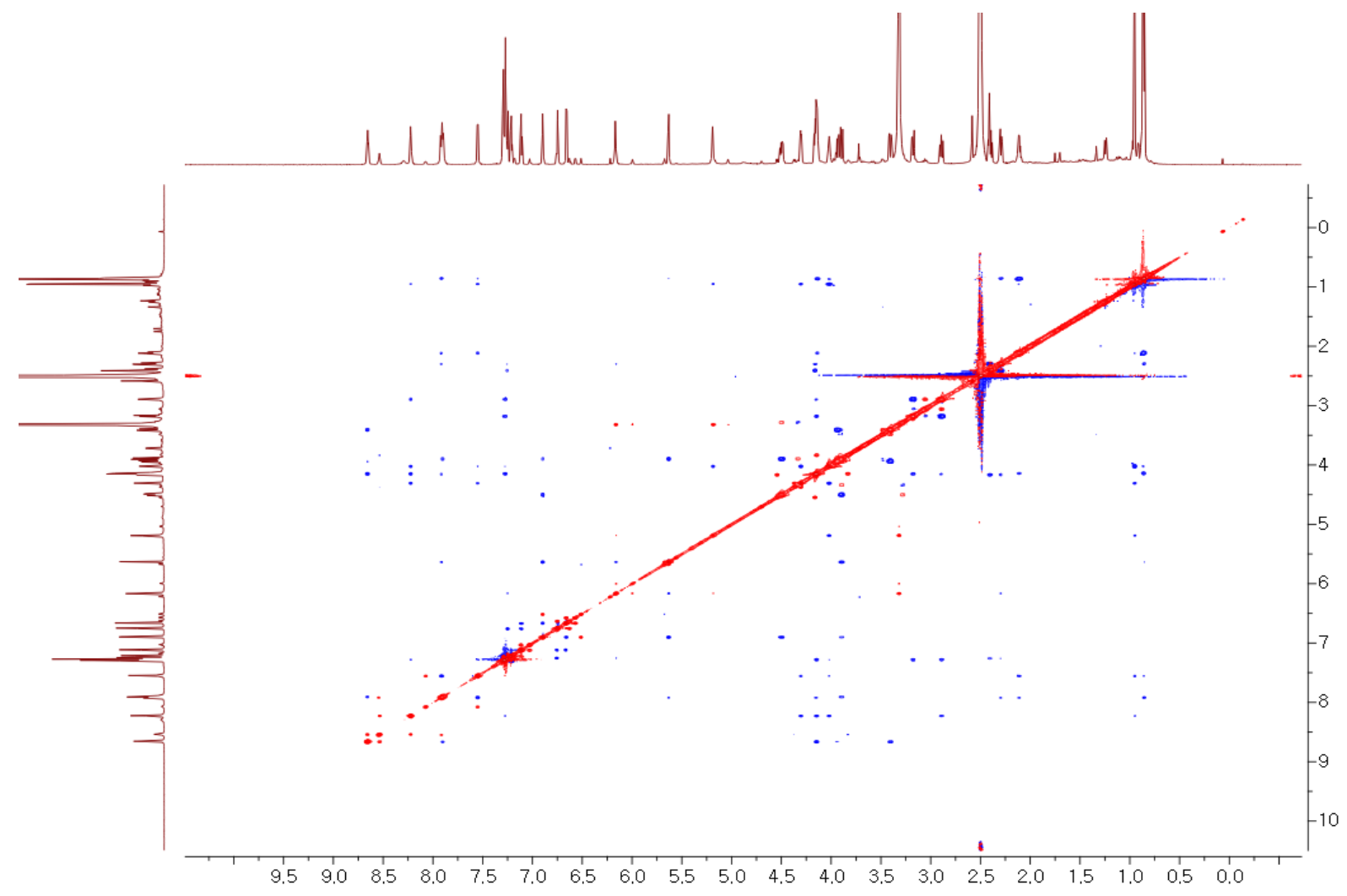

Figure S25: ROESY NMR spectrum of compound 3 (800 MHz, DMSO- $\left.d_{6}\right)$.

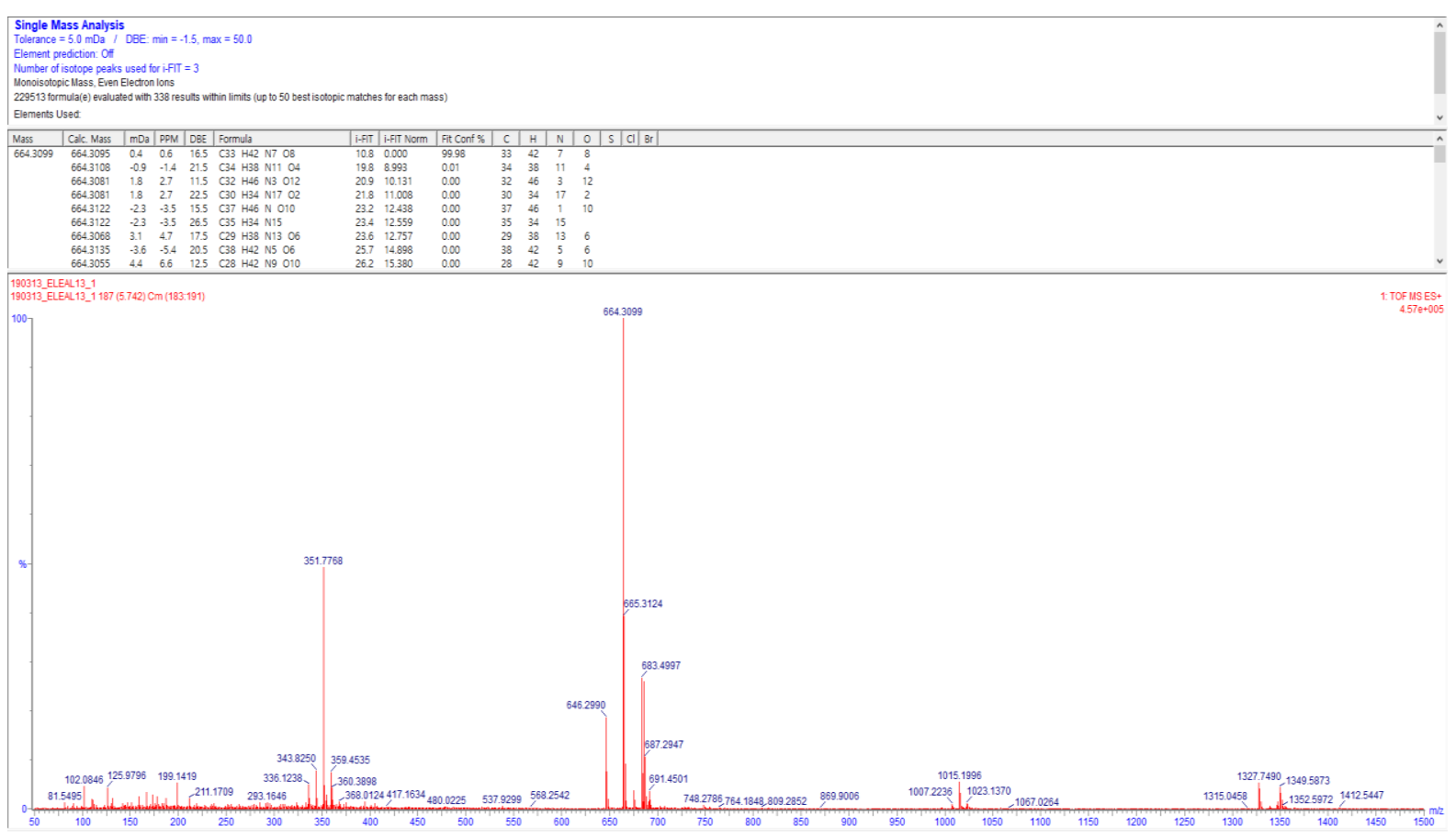

Figure S26: HR-ESIMS of compound 3. 


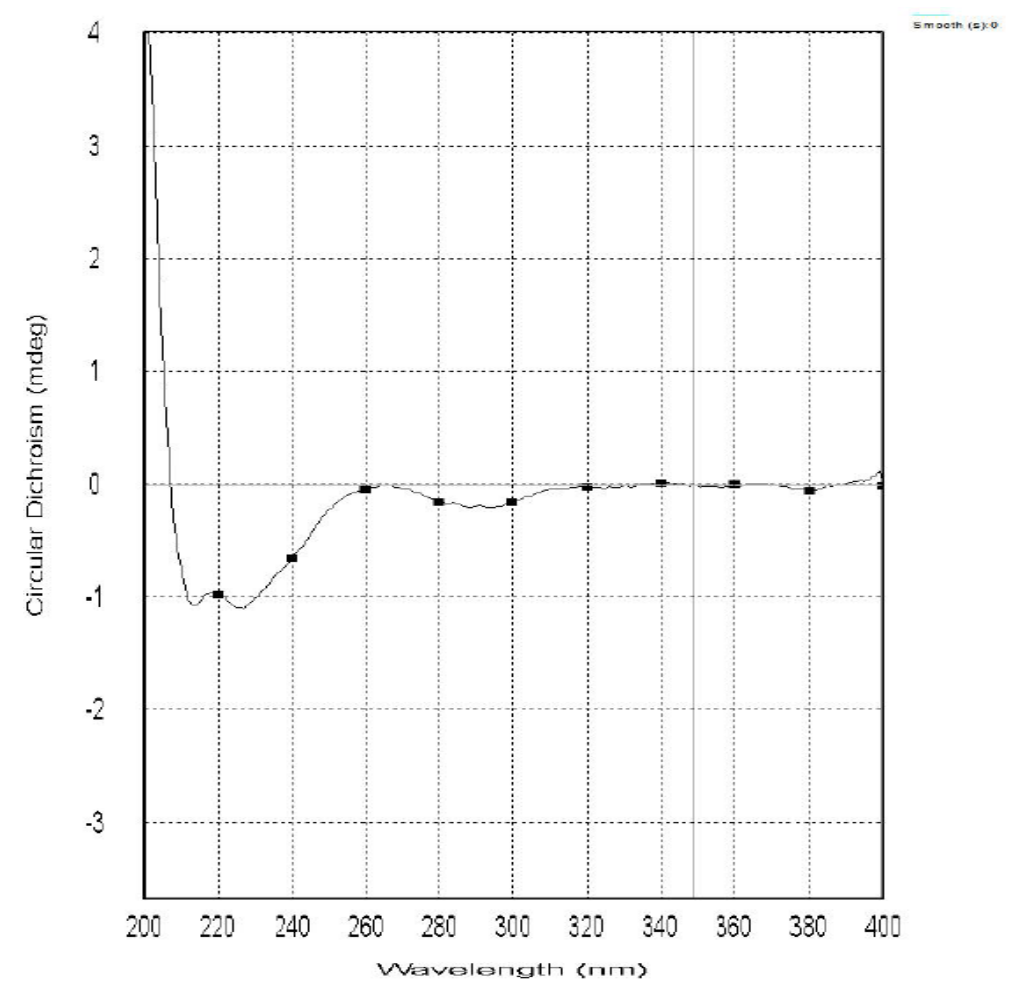

Figure S27: ECD spectrum of compound 3.

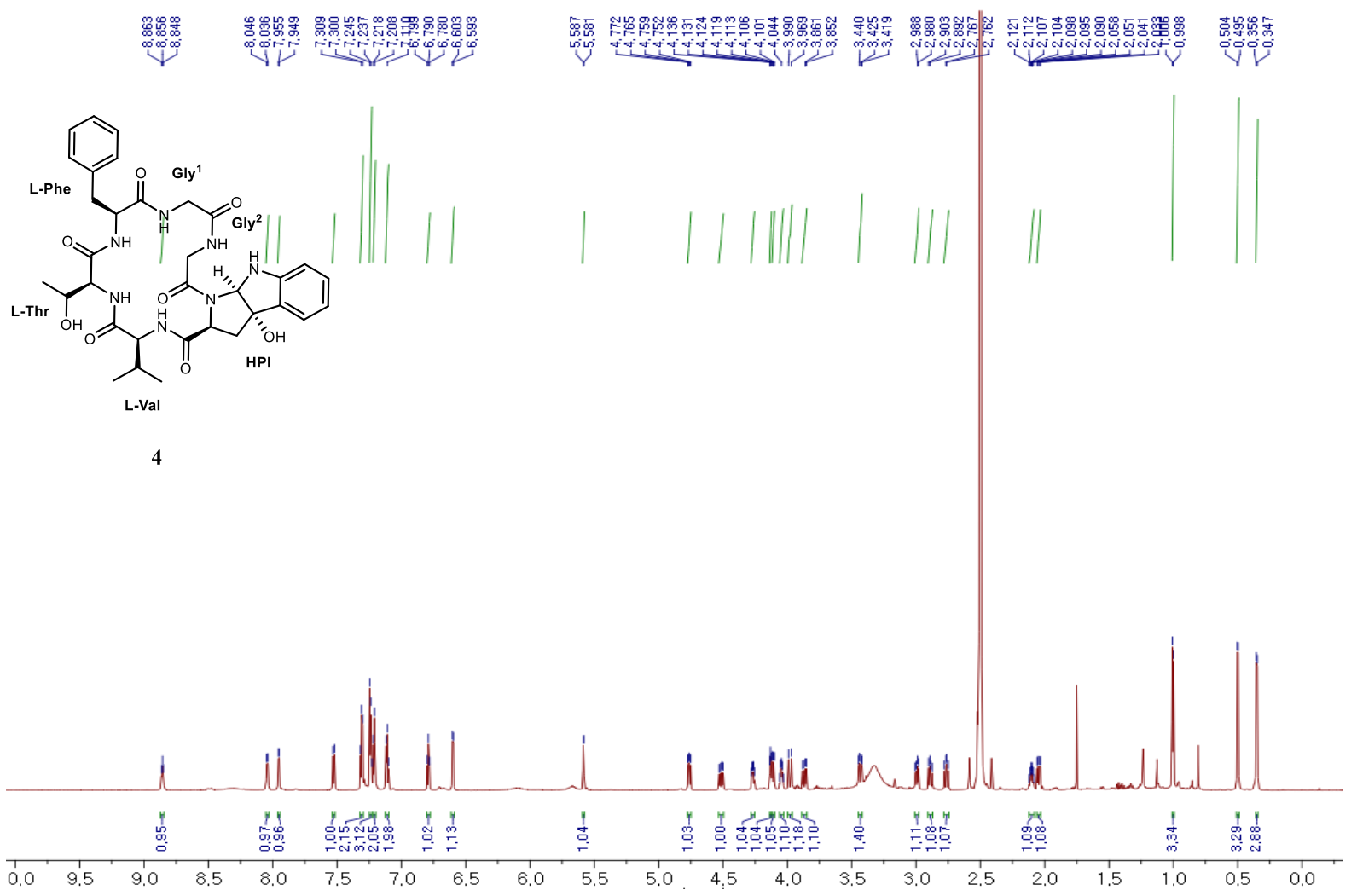

Figure S28: ${ }^{1} \mathrm{H}$ NMR spectrum of compound $4\left(800 \mathrm{MHz}, \mathrm{DMSO}-d_{6}\right)$. 


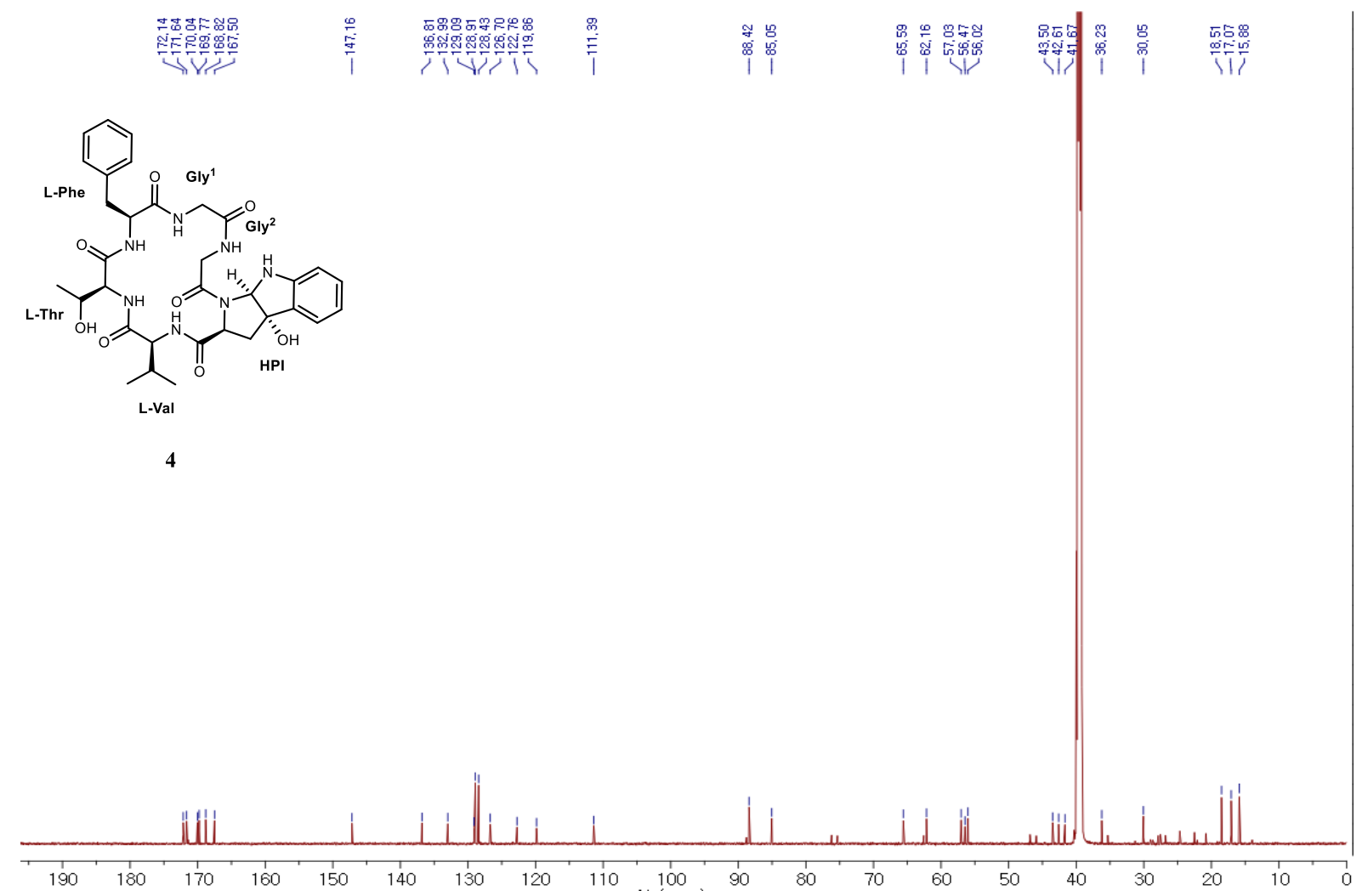

Figure S29: ${ }^{13} \mathrm{C}$ NMR spectrum of compound $4\left(800 \mathrm{MHz}, \mathrm{DMSO}-d_{6}\right)$.

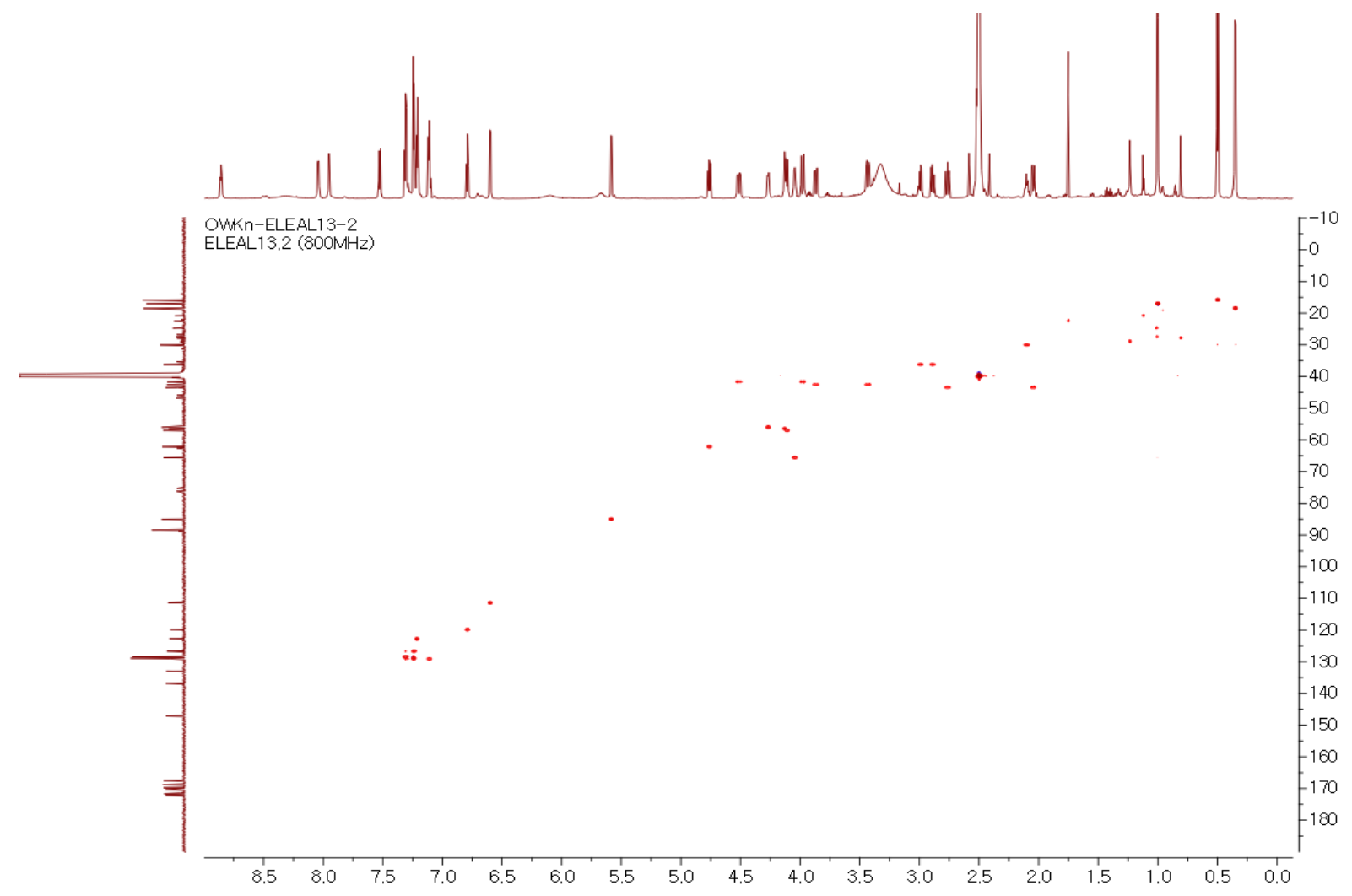

Figure S30: HSQC NMR spectrum of compound 4 (800 MHz, DMSO- $d_{6}$ ). 


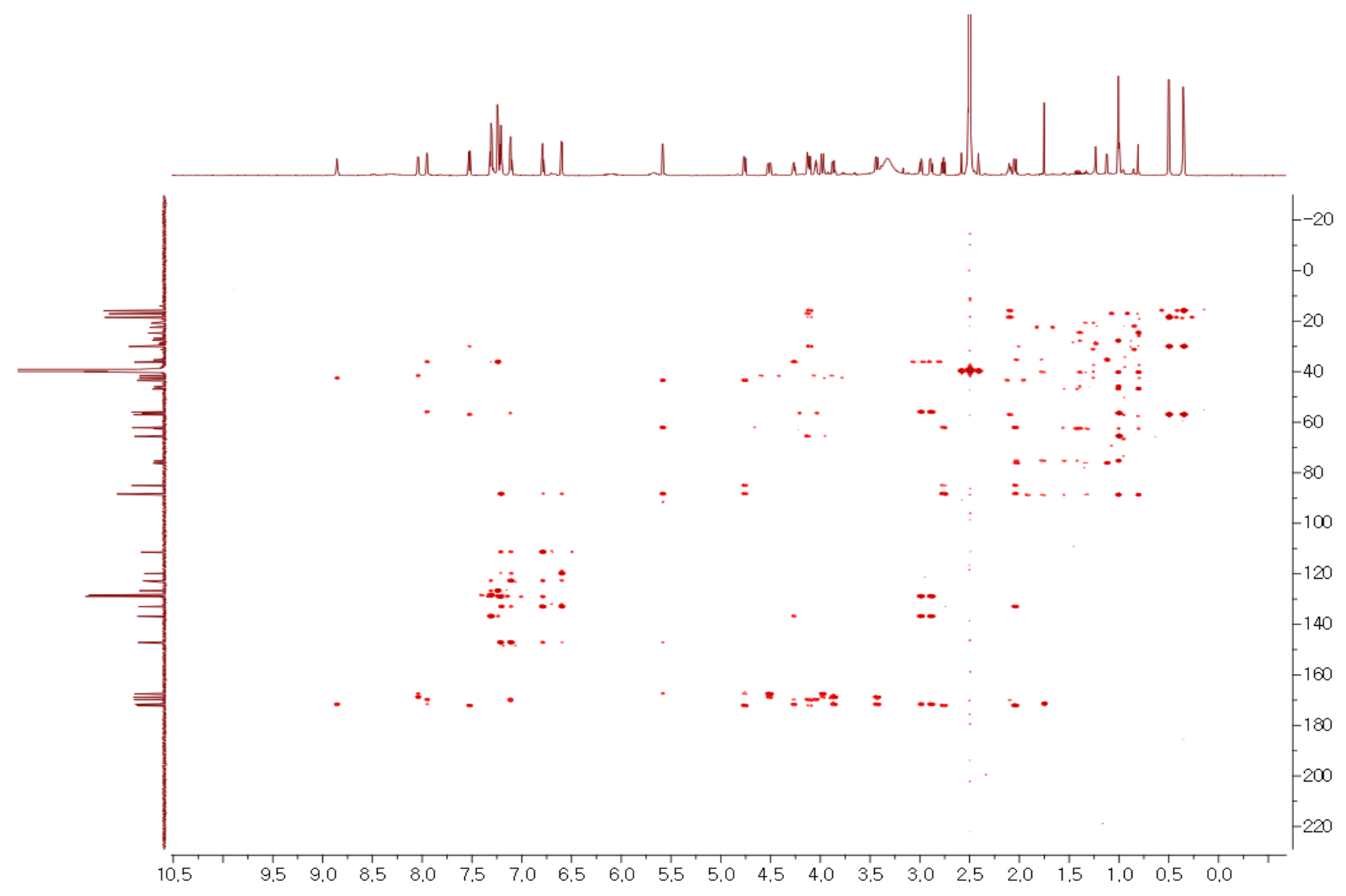

Figure S31: HMBC NMR spectrum of compound 4 (800 MHz, DMSO- $\left.d_{6}\right)$.

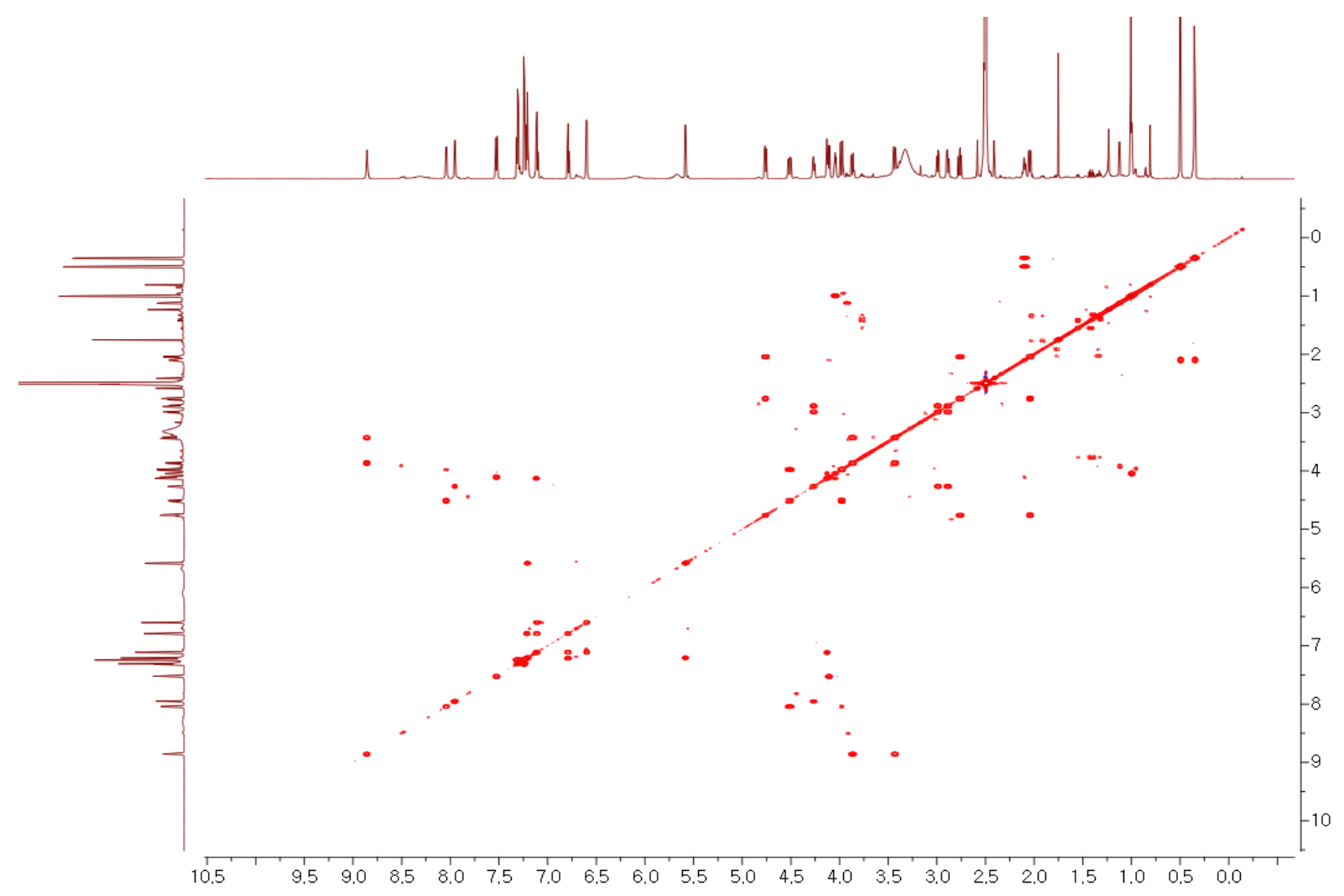

Figure S32: COSY NMR spectrum of compound 4 (800 MHz, DMSO- $\left.d_{6}\right)$. 


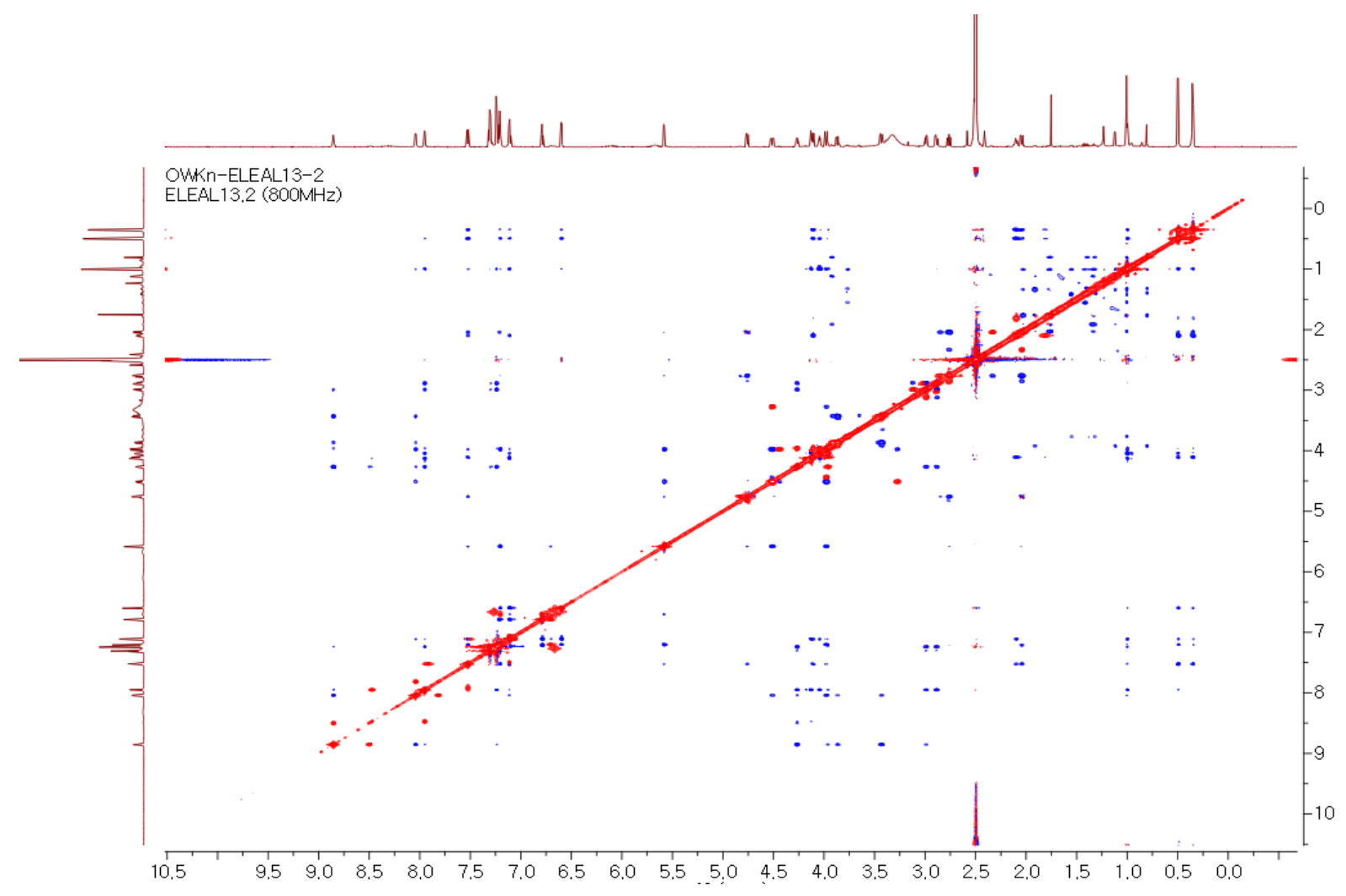

Figure S33: ROESY NMR spectrum of compound $4\left(800 \mathrm{MHz}, \mathrm{DMSO}-d_{6}\right)$.

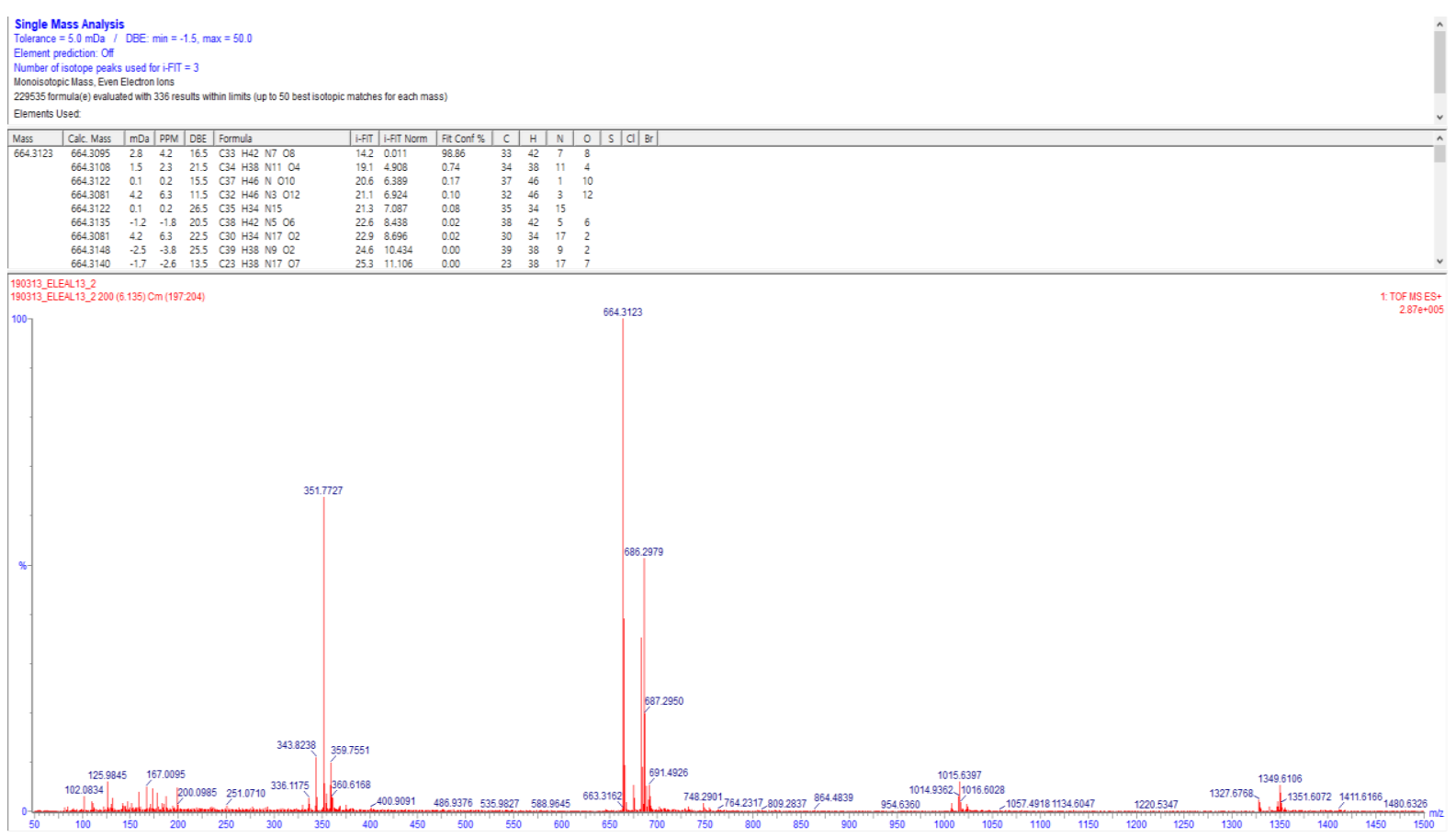

Figure S34: HR-ESIMS of compound 4. 


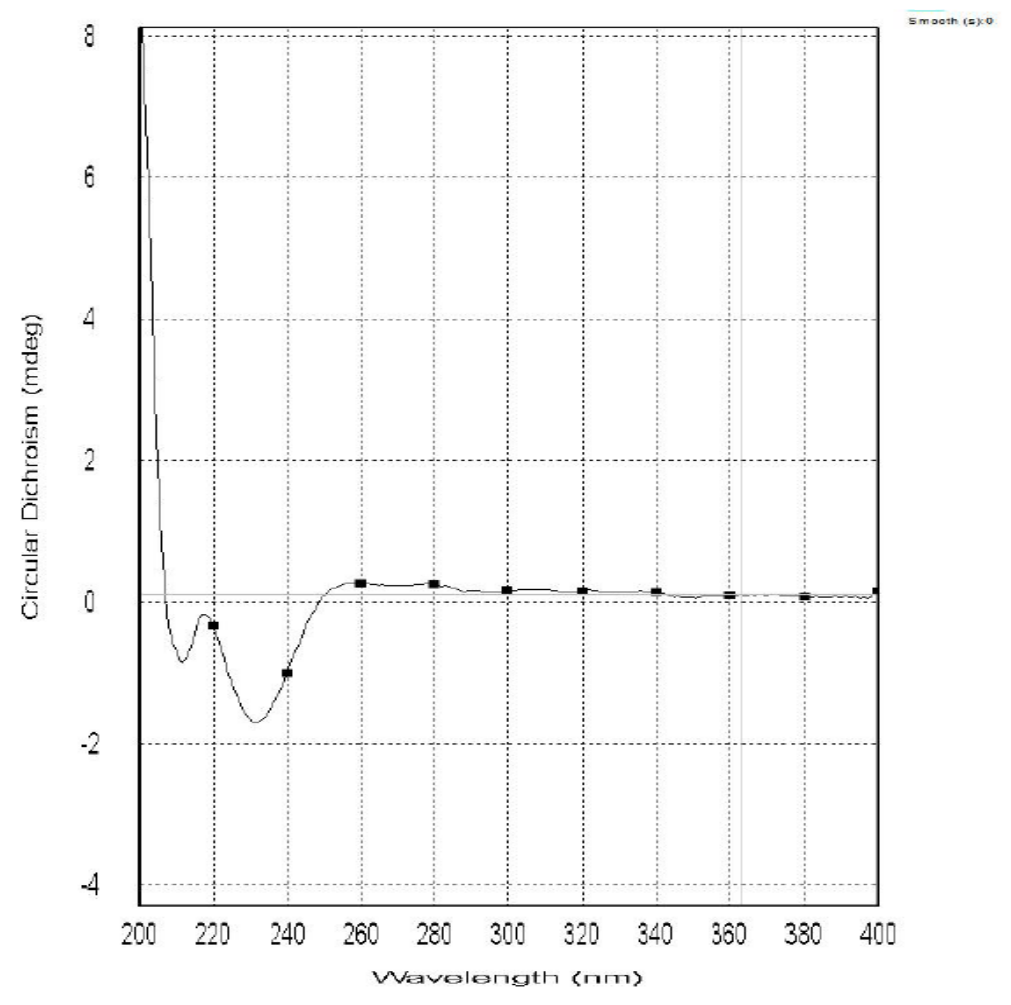

Figure S35: ECD spectrum of compound 4.

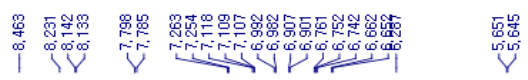

南卓

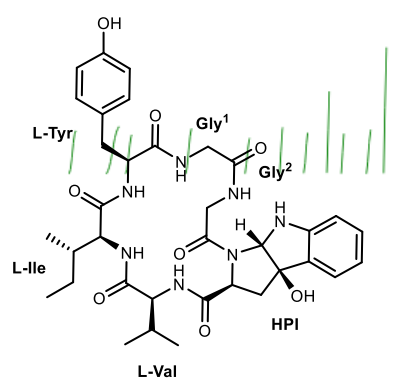

5

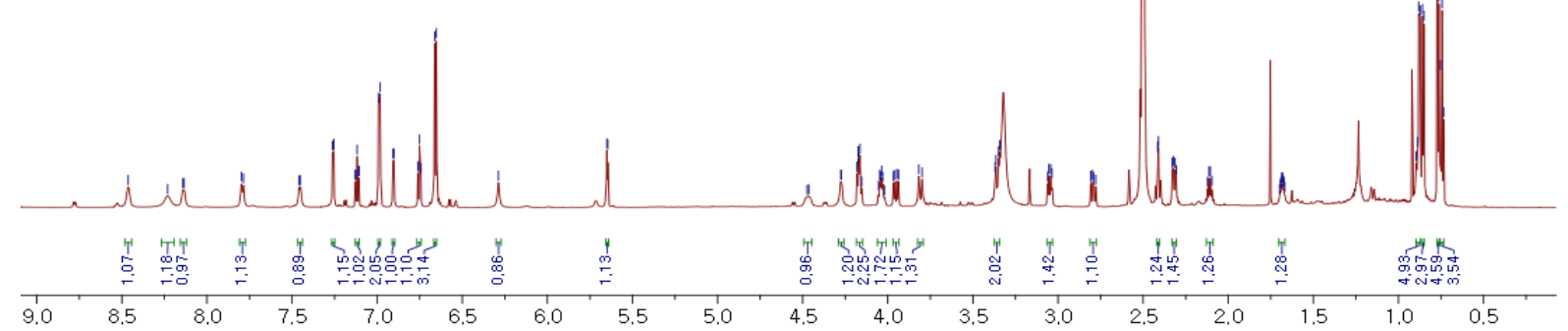

Figure S36: ${ }^{1} \mathrm{H}$ NMR spectrum of compound $5\left(800 \mathrm{MHz}, \mathrm{DMSO}-d_{6}\right)$. 


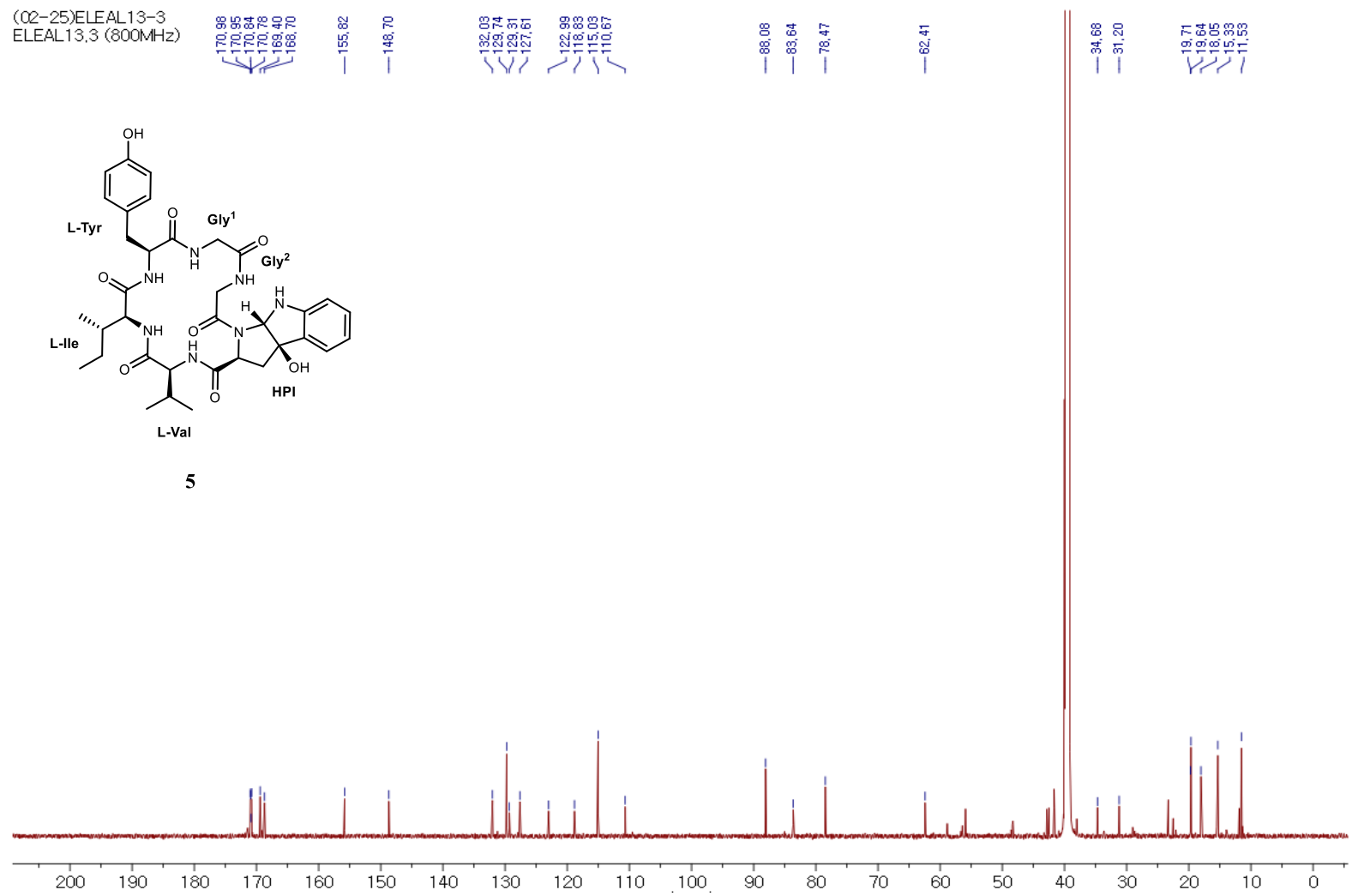

Figure S37: ${ }^{13} \mathrm{C}$ NMR spectrum of compound $5\left(800 \mathrm{MHz}, \mathrm{DMSO}-d_{6}\right)$.

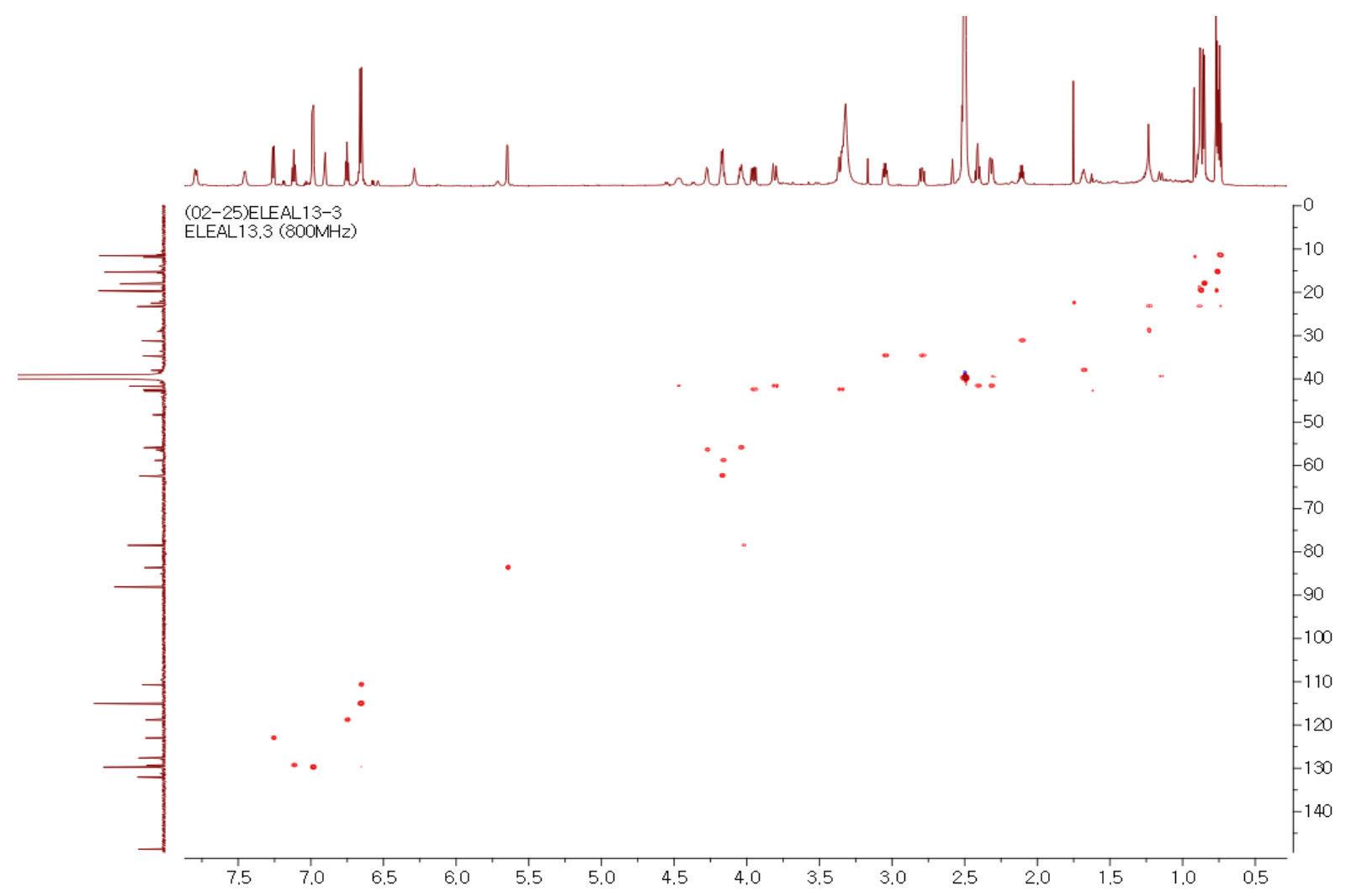

Figure S38: HSQC NMR spectrum of compound 5 ( $800 \mathrm{MHz}$, DMSO- $\left.d_{6}\right)$. 


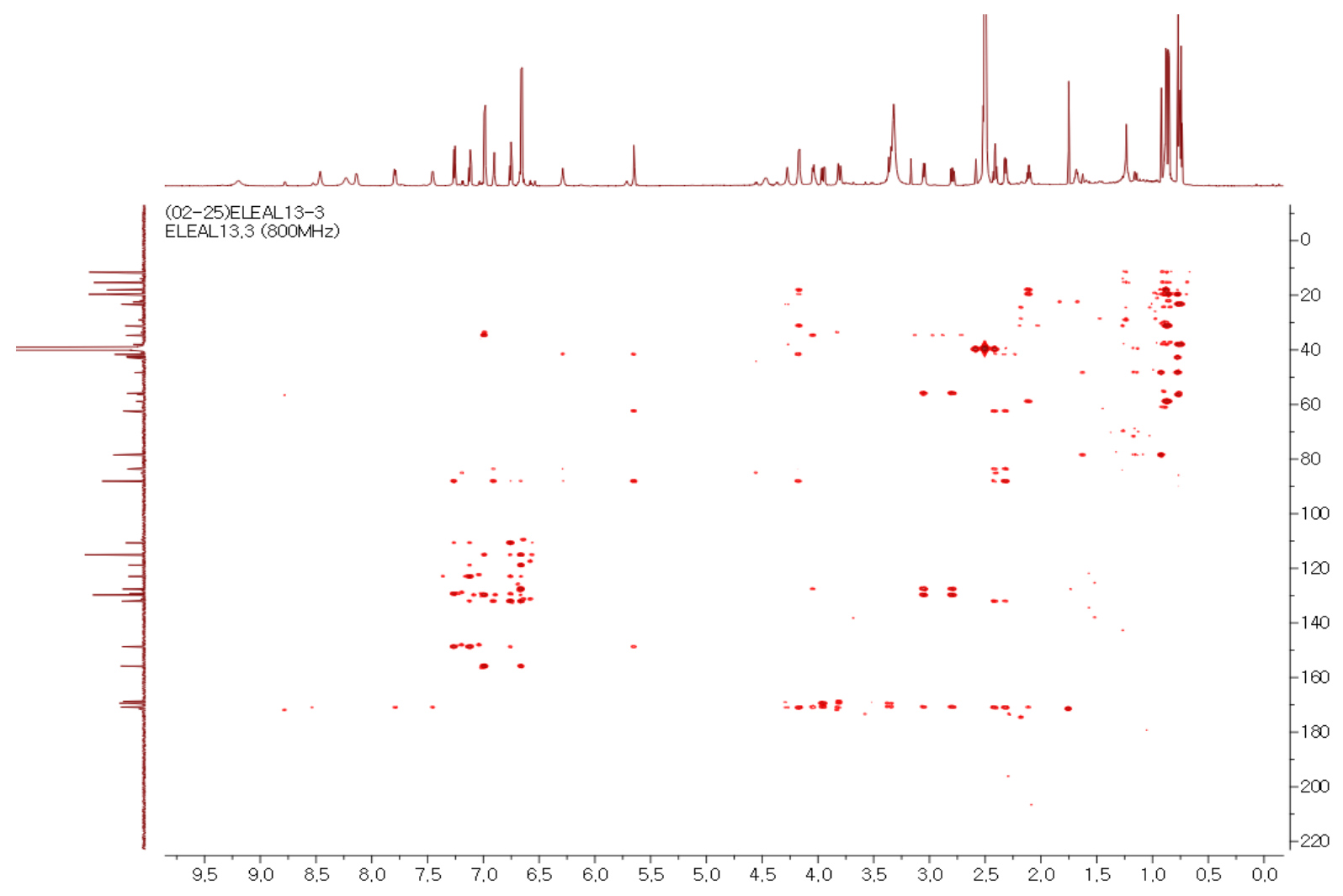

Figure S39: HMBC NMR spectrum of compound 5 (800 MHz, DMSO- $\left.d_{6}\right)$.

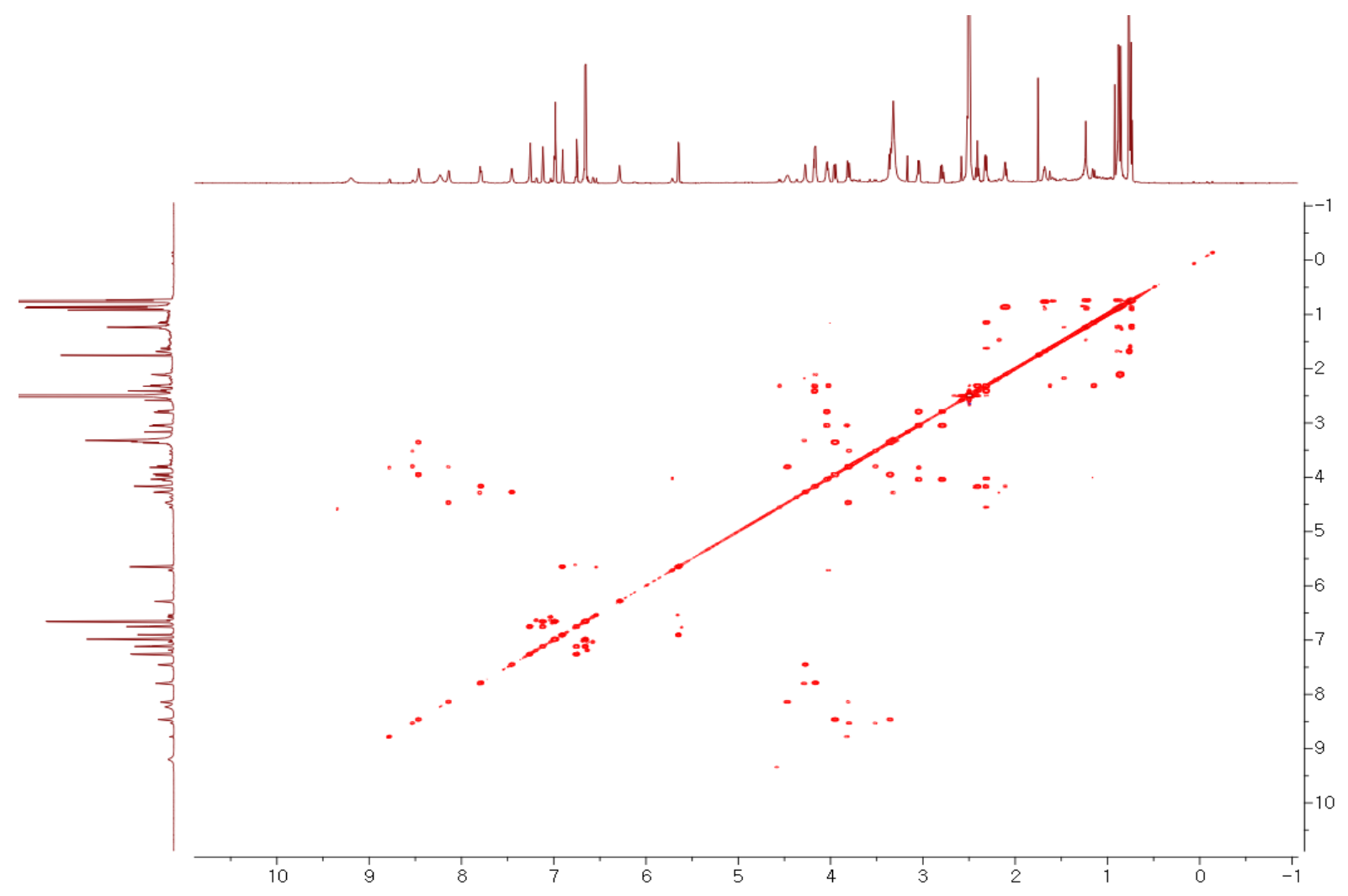

Figure S40: COSY NMR spectrum of compound 5 (800 MHz, DMSO- $\left.d_{6}\right)$. 


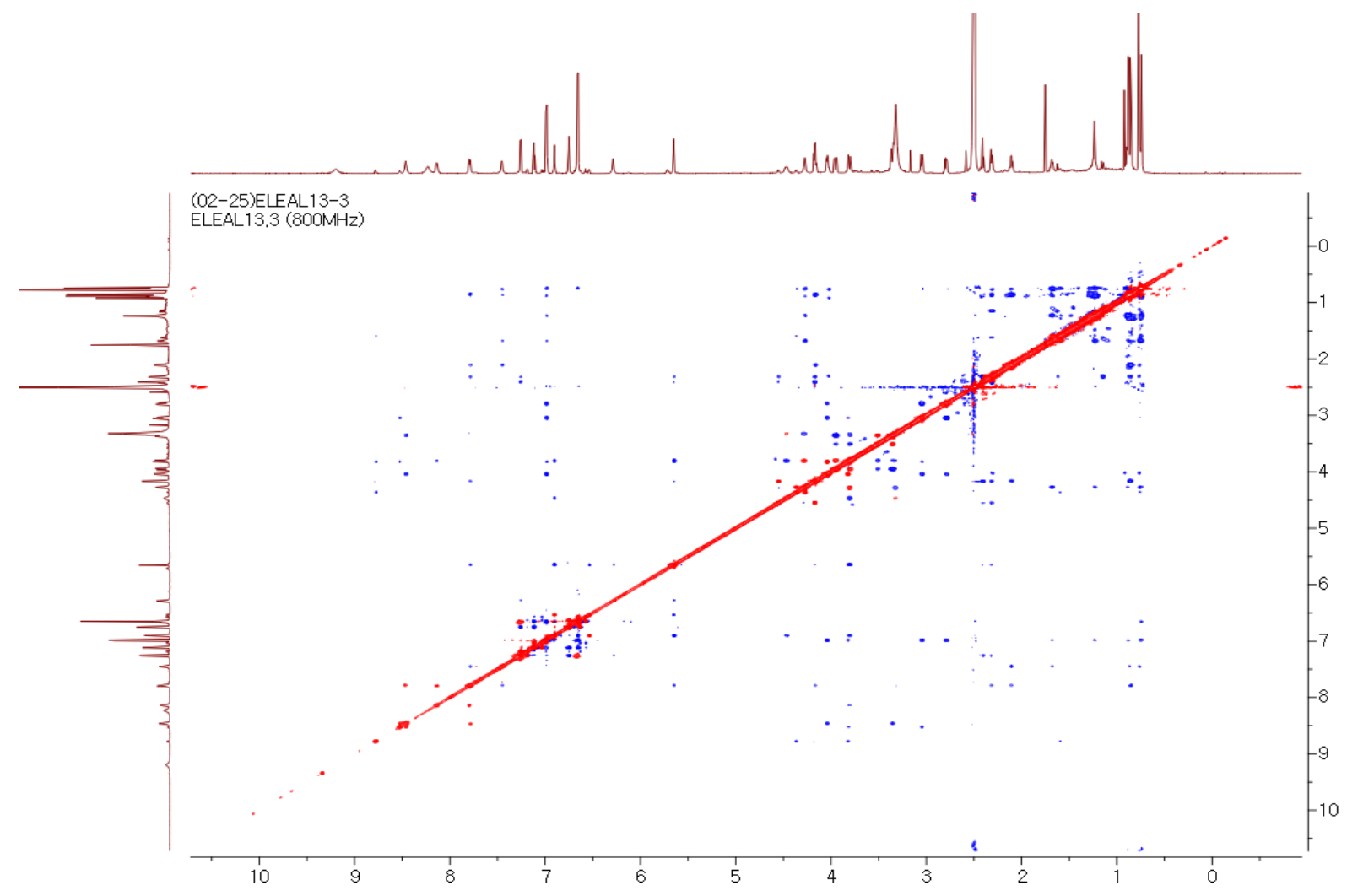

Figure S41: ROESY NMR spectrum of compound 5 (800 MHz, DMSO- $\left.d_{6}\right)$.

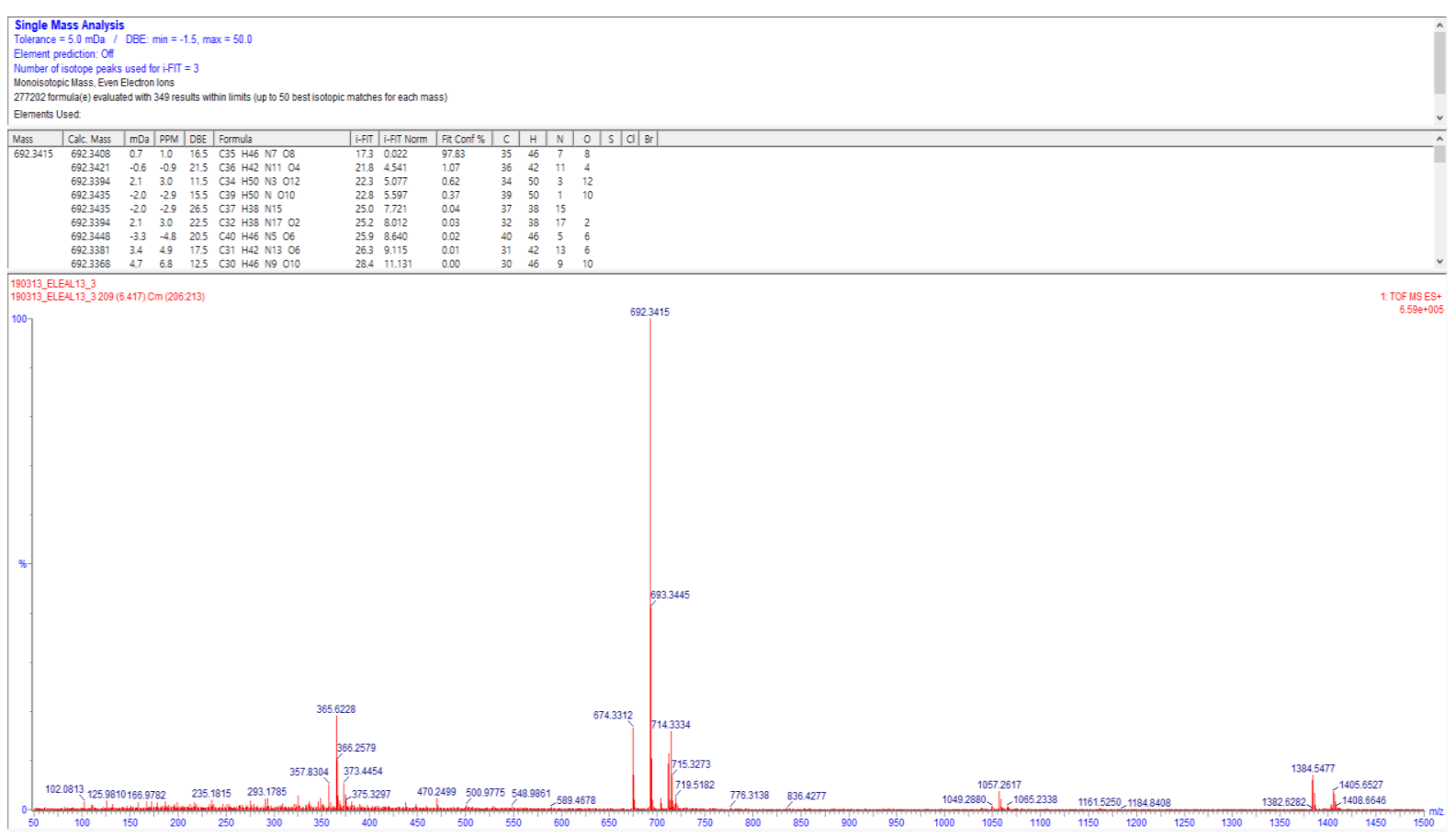

Figure S42: HR-ESIMS of compound 5. 


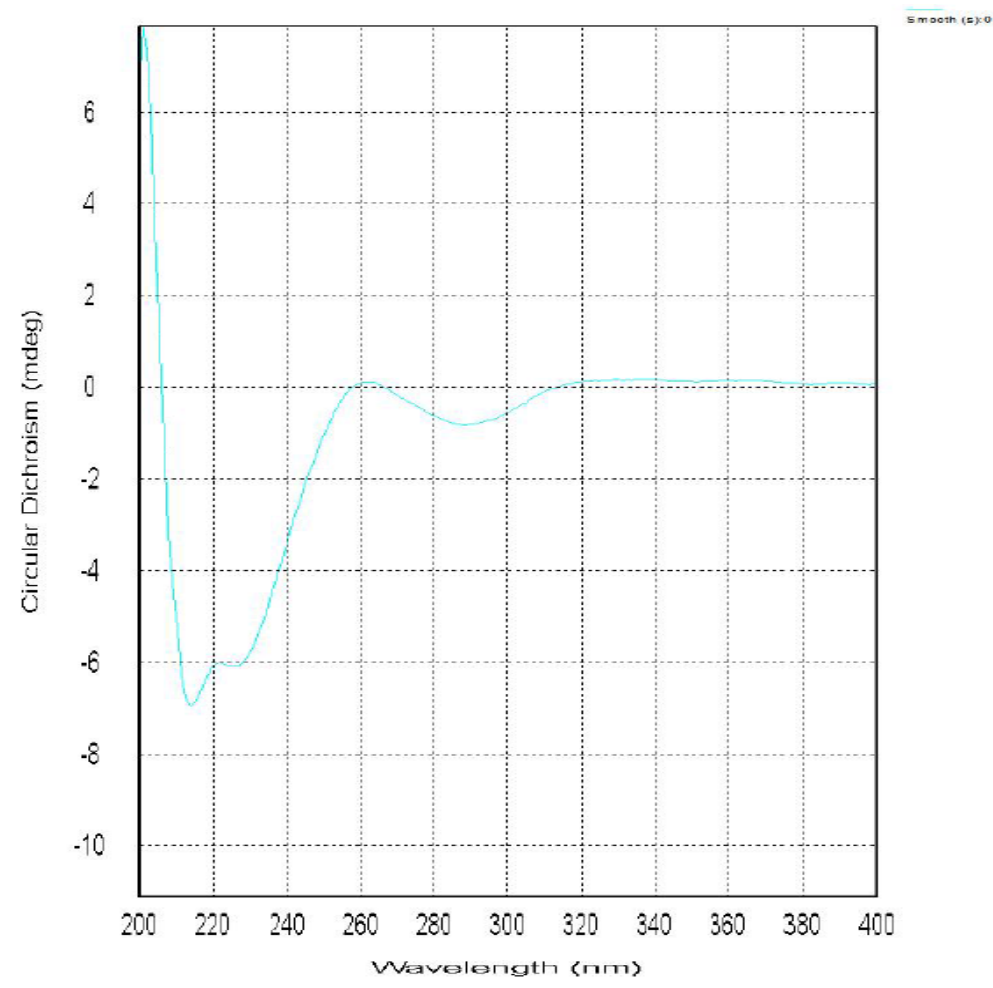

Figure S43: ECD spectrum of compound 5. 
Table S2: Comparison of retention times of derivatized amino acids in 1-5 to those of authentic amino acids.

\begin{tabular}{|c|c|c|c|c|c|c|c|c|c|c|c|c|c|c|c|c|c|c|}
\hline \multirow{2}{*}{$\begin{array}{l}\text { Retention } \\
\text { time (min) }\end{array}$} & \multicolumn{2}{|c|}{ Leu } & \multicolumn{2}{|c|}{ Val } & \multicolumn{2}{|c|}{ Pro } & \multicolumn{2}{|c|}{ Tyr } & \multicolumn{2}{|c|}{ Phe } & \multicolumn{4}{|c|}{ Thr } & \multicolumn{4}{|c|}{ Ile } \\
\hline & $\mathbf{L}$ & D & $\mathbf{L}$ & D & $\mathbf{L}$ & D & $\mathbf{L}$ & D & $\mathbf{L}$ & D & $\mathbf{L}$ & D & L-allo & D-allo & $\mathbf{L}$ & D & L-allo & D-allo \\
\hline Authentic & 24.3 & 30.3 & 21.7 & 27.2 & 18.4 & 20.9 & 19.7 & 21.2 & 24.7 & 29.1 & $17.1^{a}$ & 18.5 & 17.4 & 18.0 & 37.3 & 50.2 & 36.9 & 50.2 \\
\hline Authentic & & & & & & & & & & & $17.2^{b}$ & 18.5 & 17.5 & 17.9 & & & & \\
\hline 1 & 24.4 & & 21.5 & & 18.6 & & & & & & $17.1^{a}$ & & & & & & & \\
\hline 2 & 24.0 & & & & 18.2 & & 19.5 & & & & & & & & 37.3 & & & \\
\hline 3 & & & 21.6 & & & & & & 24.7 & & $17.2^{b}$ & & & & & & & \\
\hline 4 & & & 21.2 & & & & & & 24.7 & & $17.2^{b}$ & & & & & & & \\
\hline 5 & & & 21.4 & & & & 19.5 & & & & & & & & 37.3 & & & \\
\hline
\end{tabular}

$\overline{a, b}$ They were measured in the same sequence and time. 


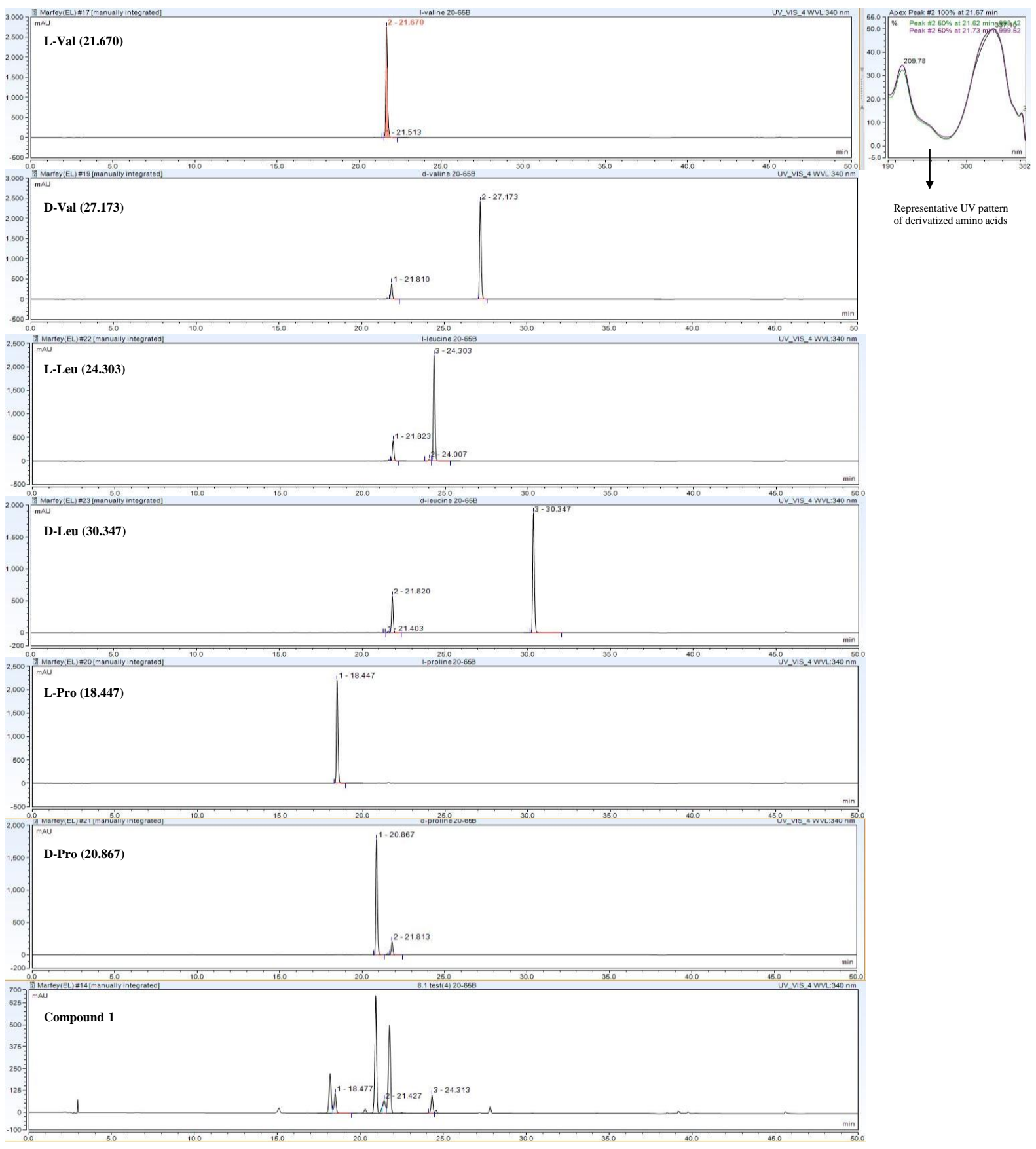

Figure S44: Advanced Marfey's analysis of compound 1 for common amino acids. 


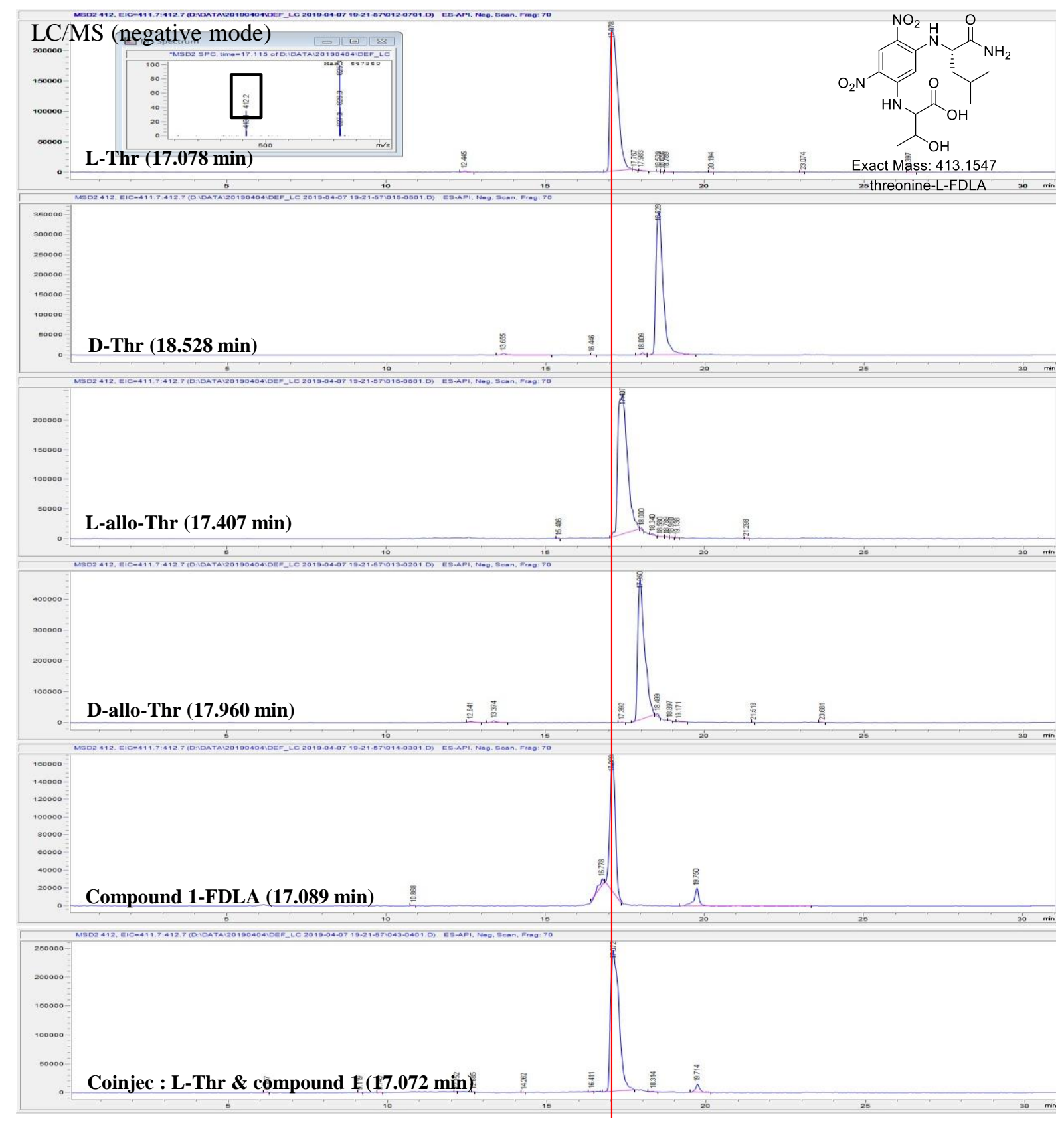

Figure S45: Advanced Marfey's analysis of compound 1 for Thr. 

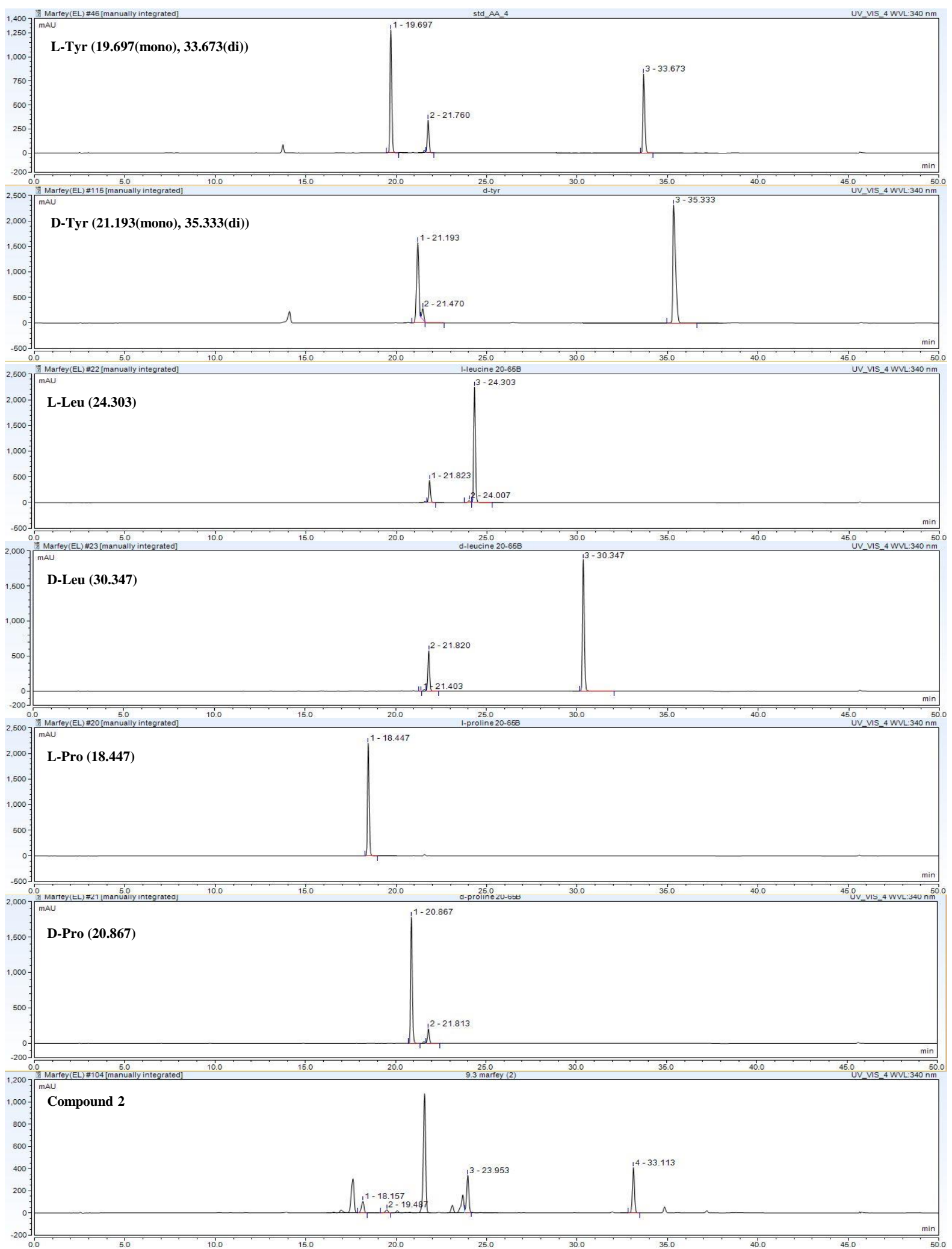

Figure S46: Advanced Marfey's analysis of compound 2 for common amino acids. 


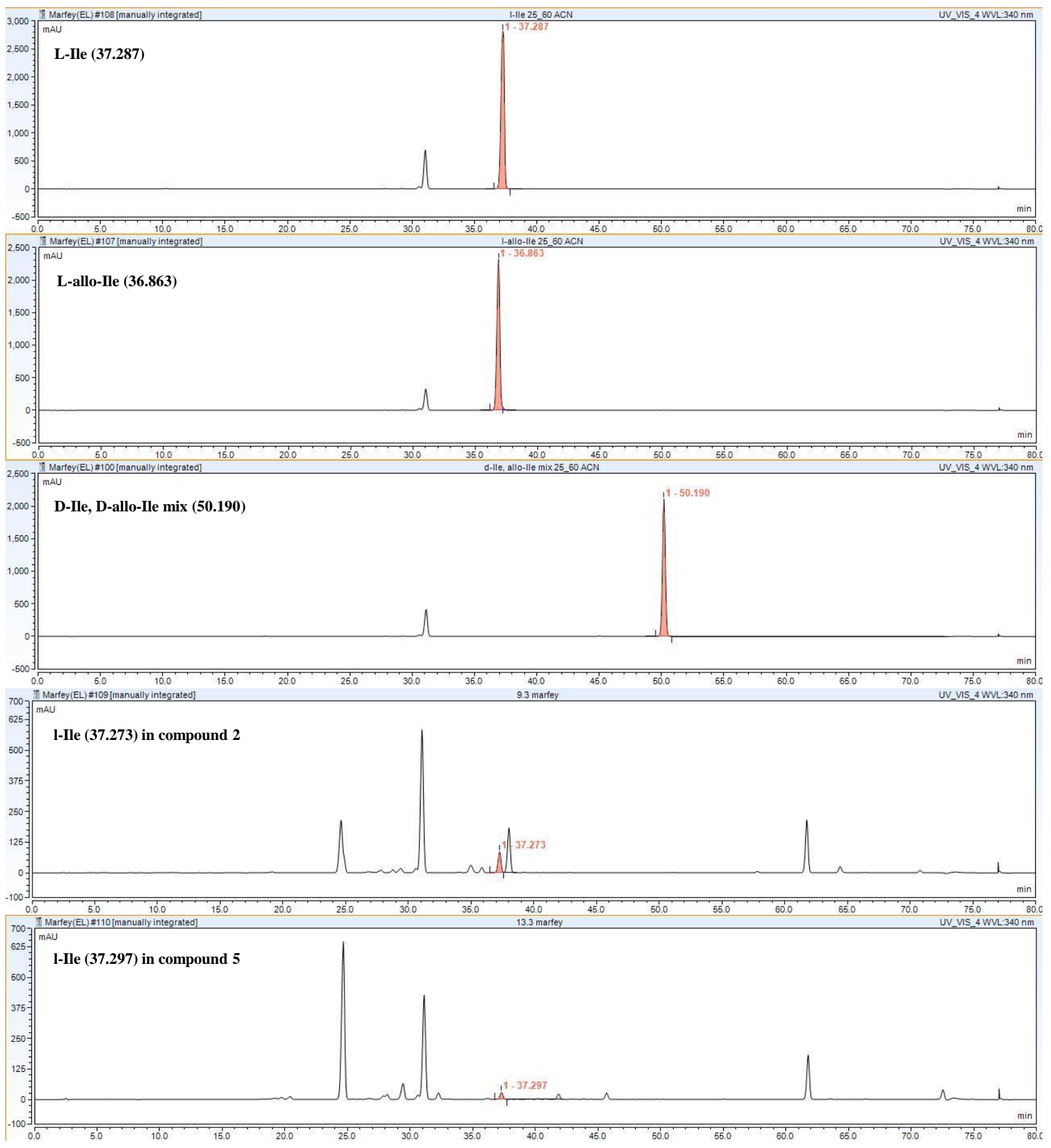

Figure S47: Advanced Marfey's analysis of compounds 2 and 5 for Ile. 


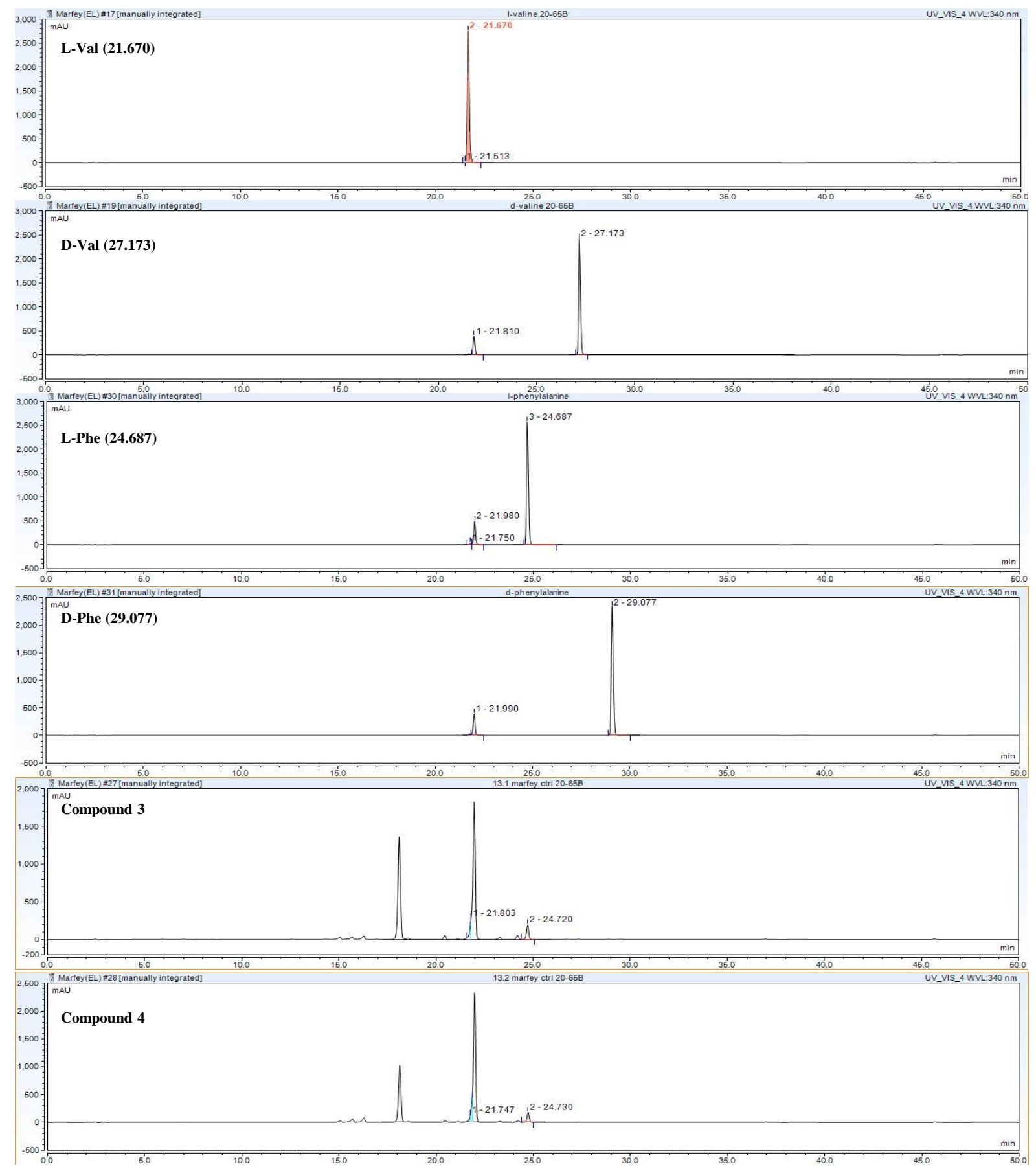

Figure S48: Advanced Marfey's analysis of compounds $\mathbf{3}$ and $\mathbf{4}$ for common amino acids. 


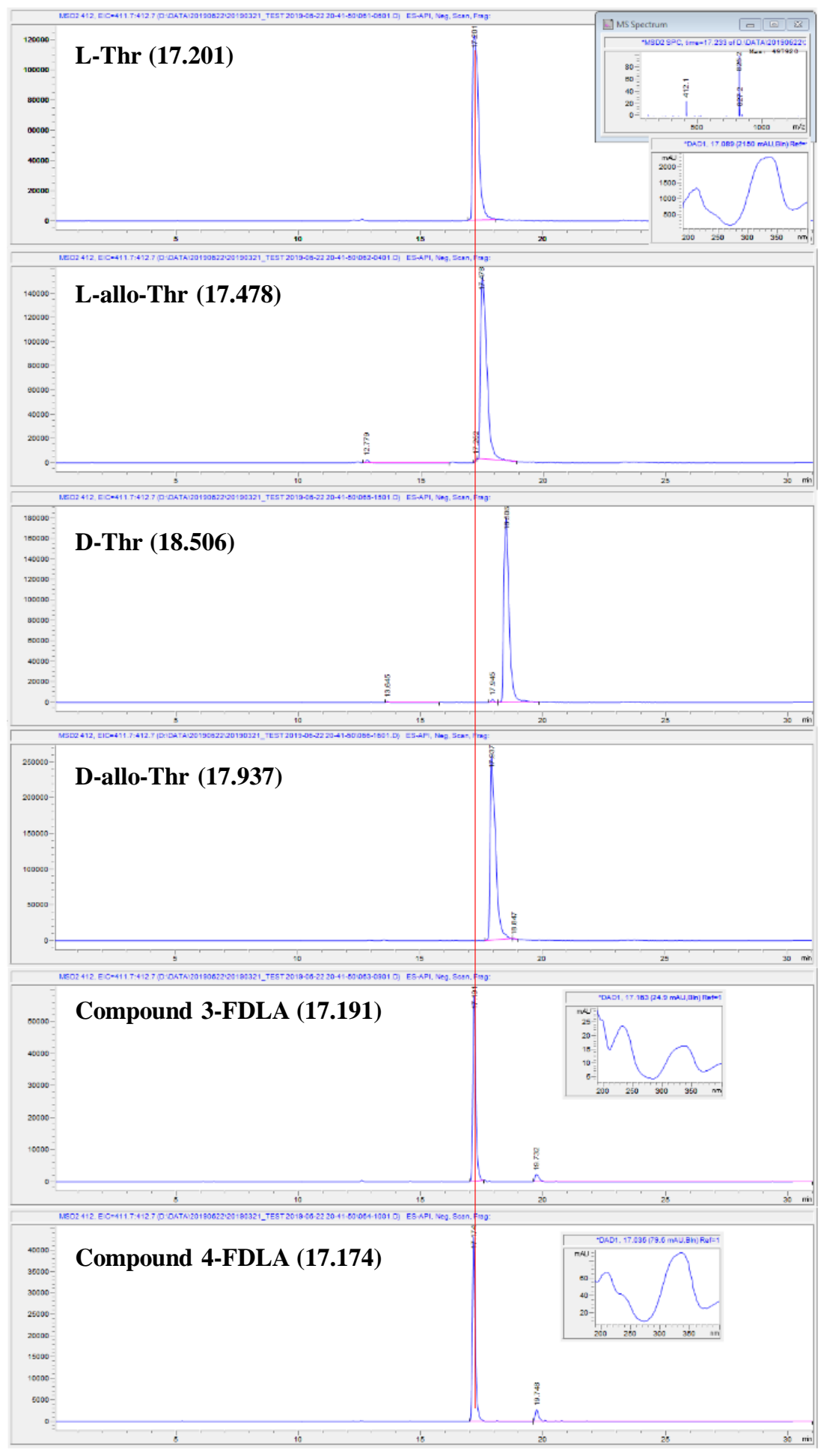

Figure S49: Advanced Marfey's analysis of compounds 3 and $\mathbf{4}$ for Thr. 

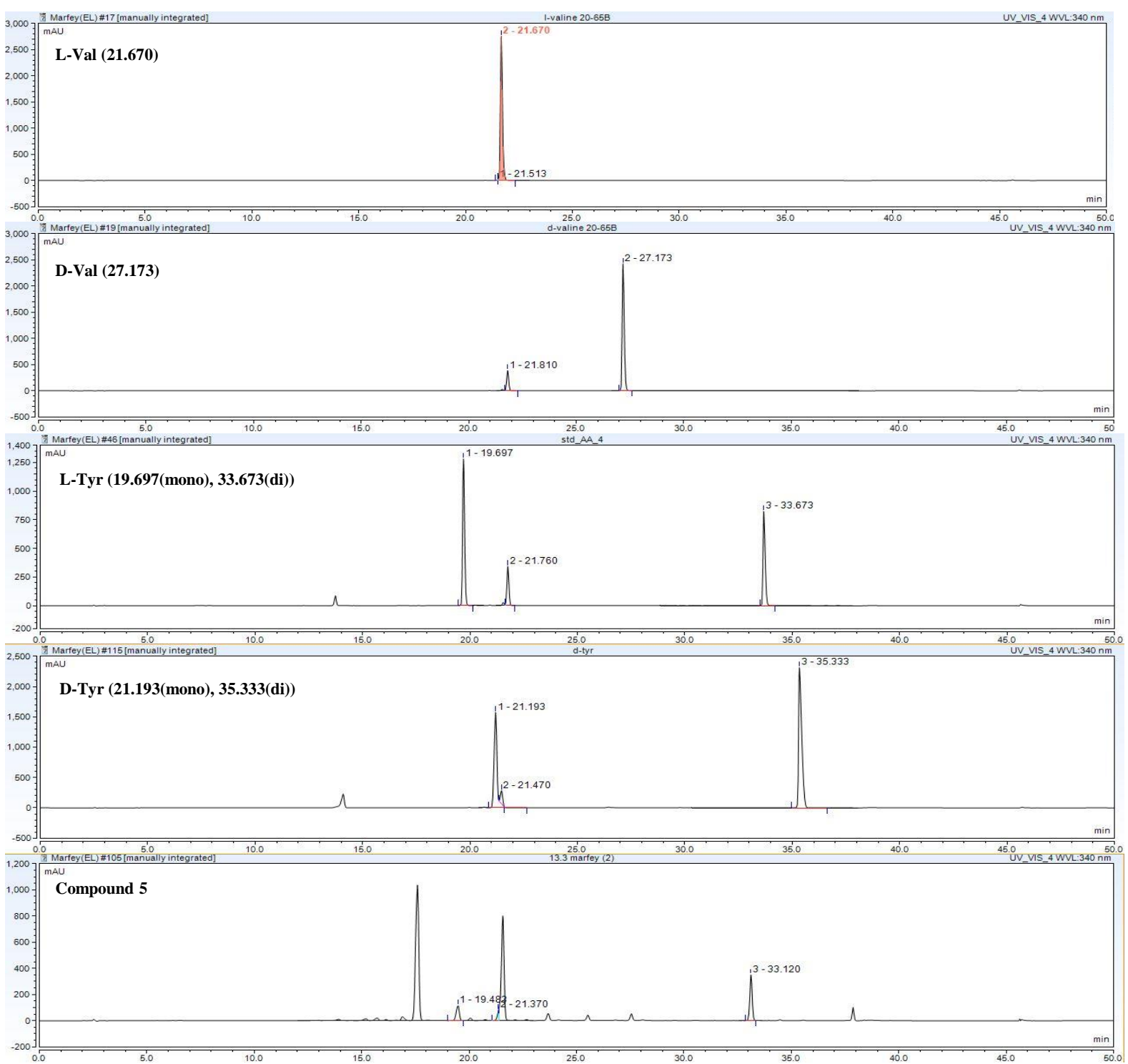

Figure S50: Advanced Marfey's analysis of compound 5 for common amino acids. 


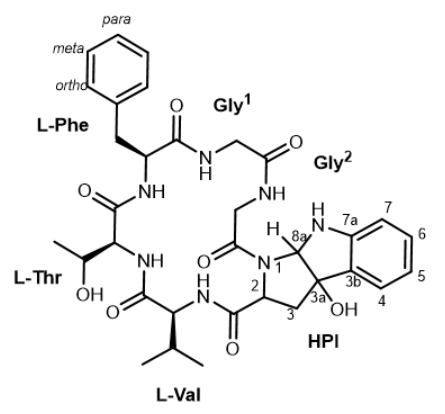

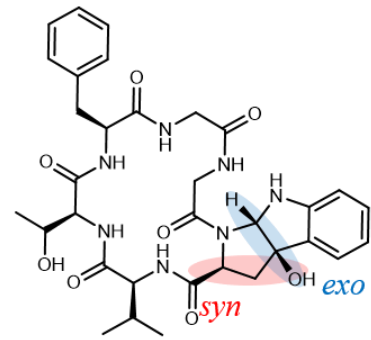
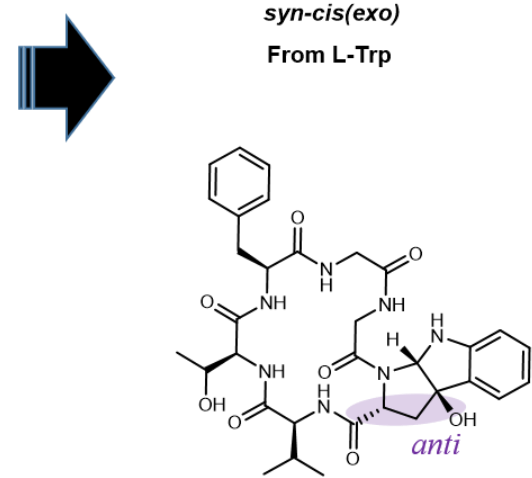

anti-cis(exo)

From D-Trp

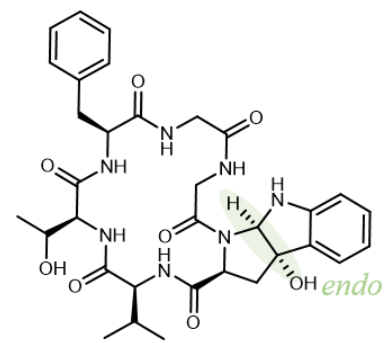

anti-cis(endo)

From L-Trp

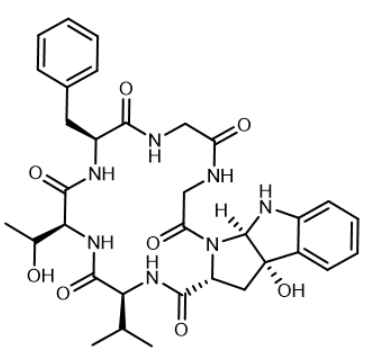

syn-cis(endo)

From D-Trp

Figure S51: Four possible configurations of HPI moiety. 


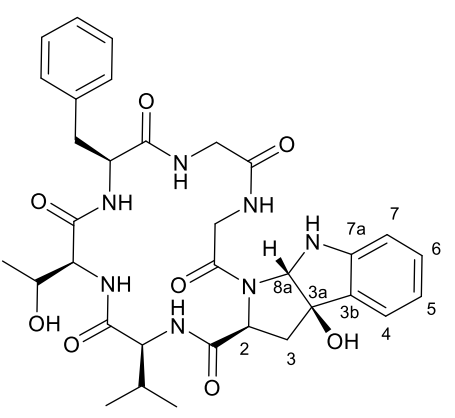

syn-cis(exo) Compound 3

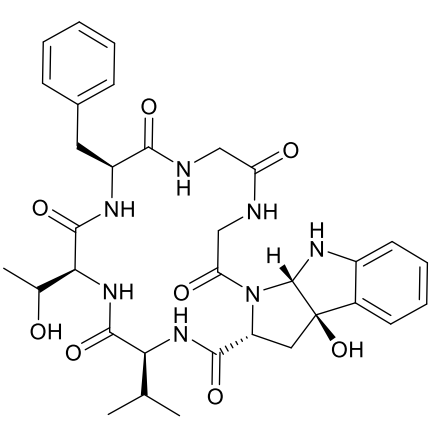

$\operatorname{anti-cis(exo)}$
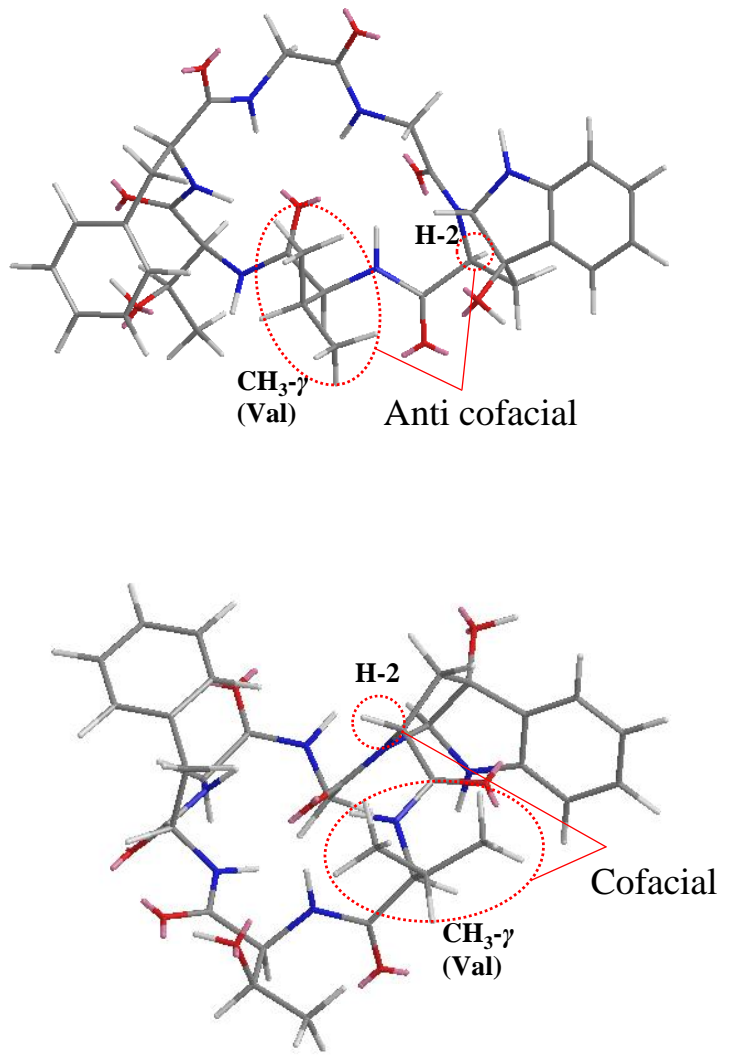
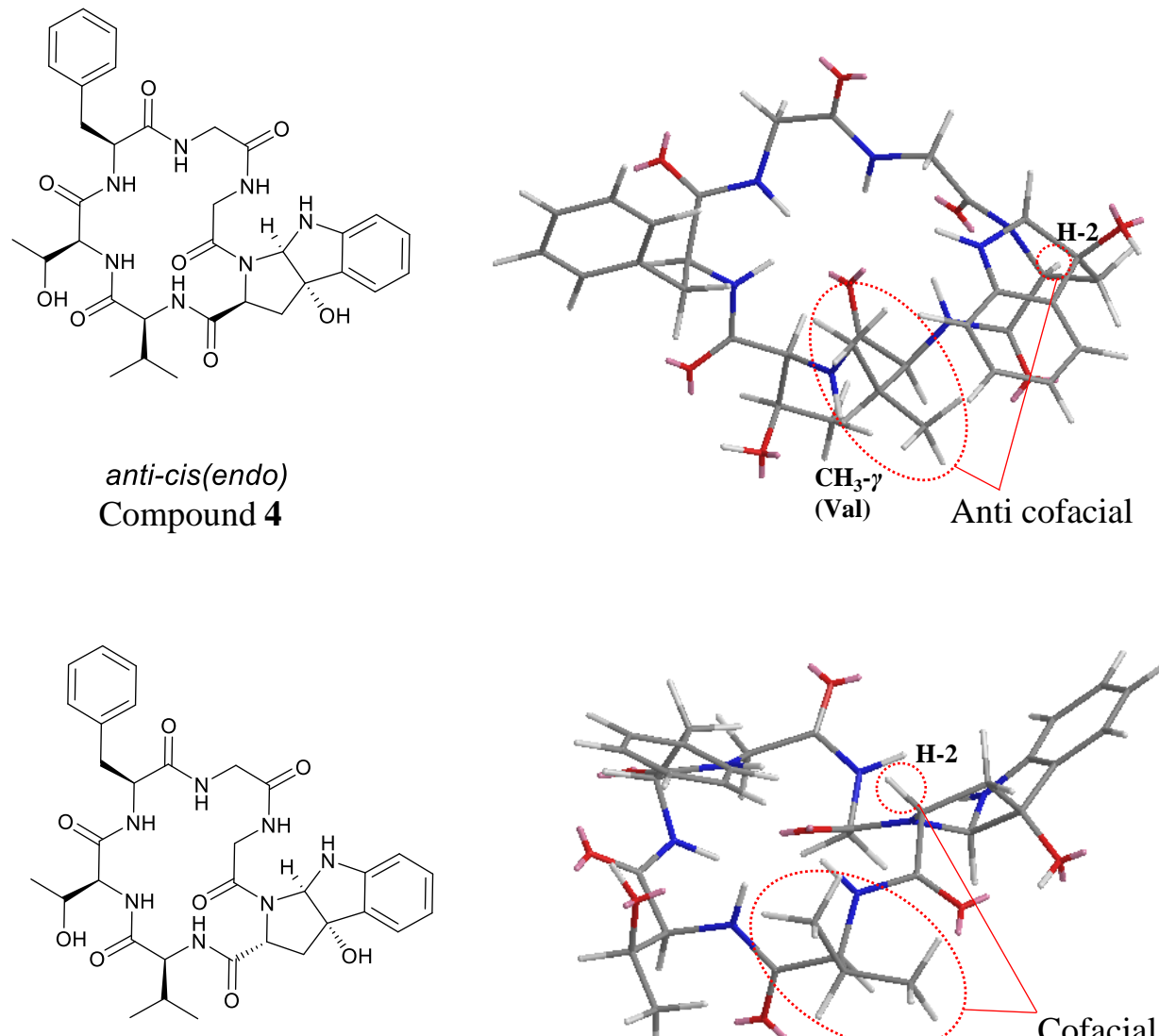

syn-cis(endo)

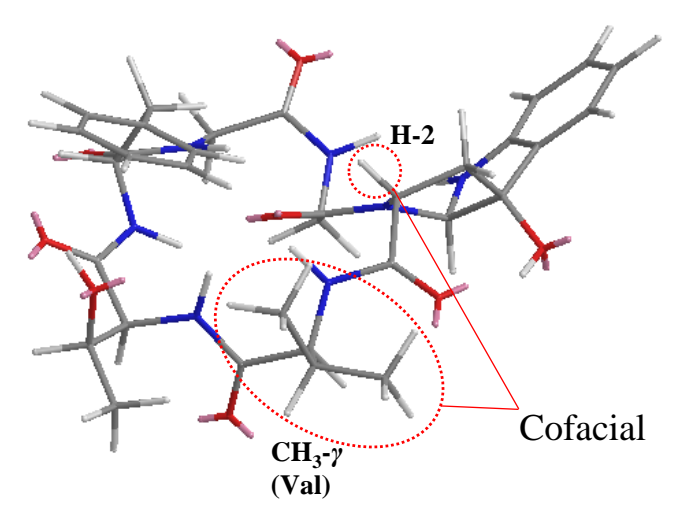

Figure S52: Distance between $\mathrm{CH}_{3}-\gamma$ (Val) and $\mathrm{H}-2$ in 3D structures of four possible configurations of HPI. 

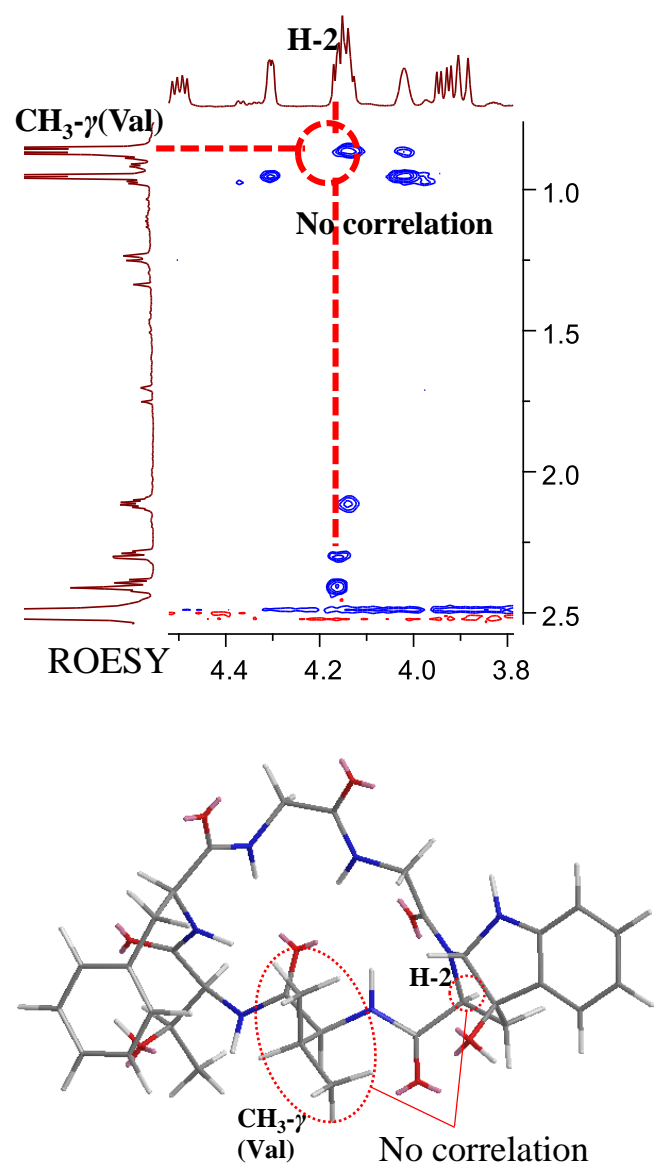

syn-cis(exo)

Compound $\mathbf{3}$
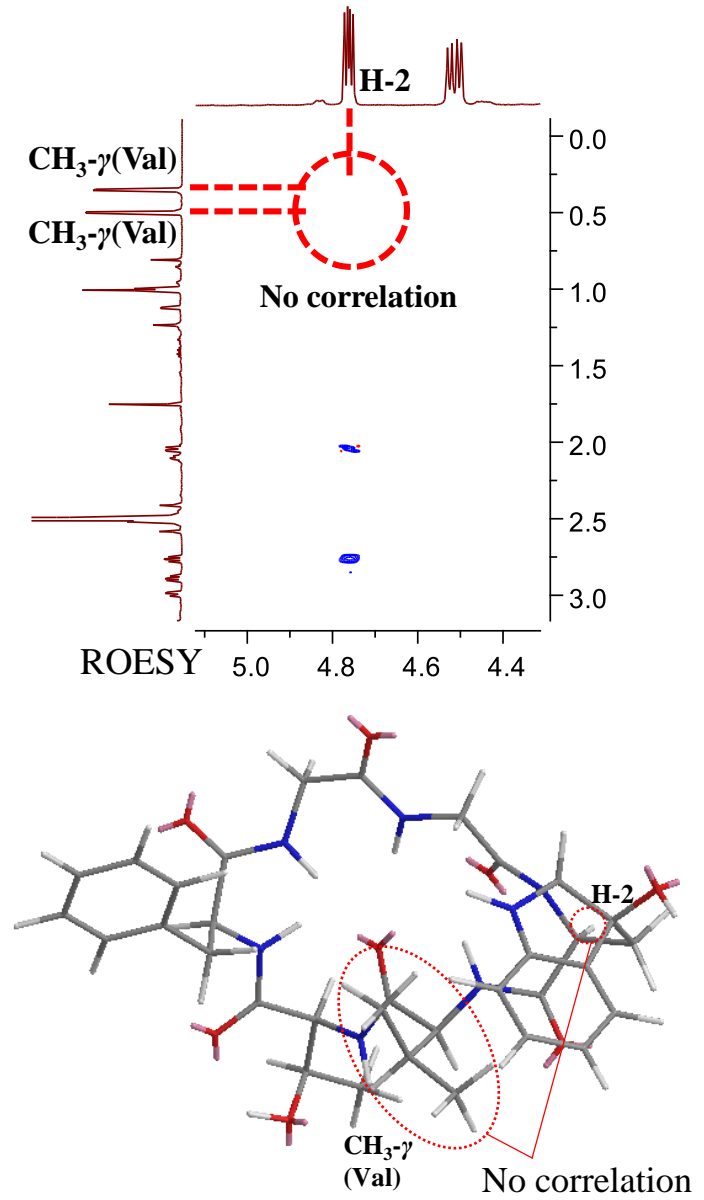

anti-cis(endo)

Compound 4

Figure S53: Expanded ROESY spectrum for correlations between $\mathrm{CH}_{3}-\gamma$ (Val) and $\mathrm{H}-2$. 


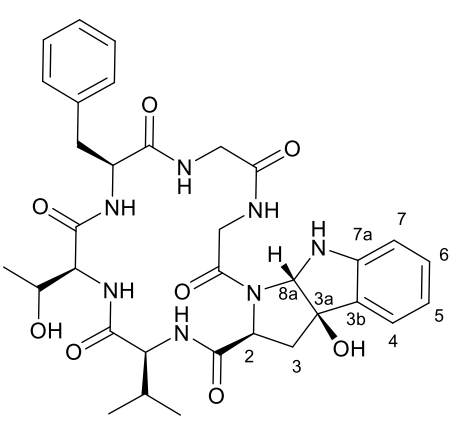

syn-cis(exo) Compound 3

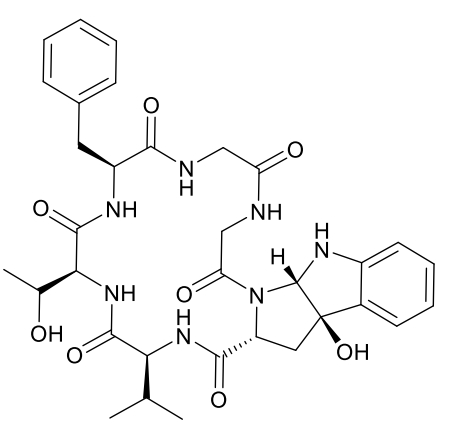

anti-cis(exo)
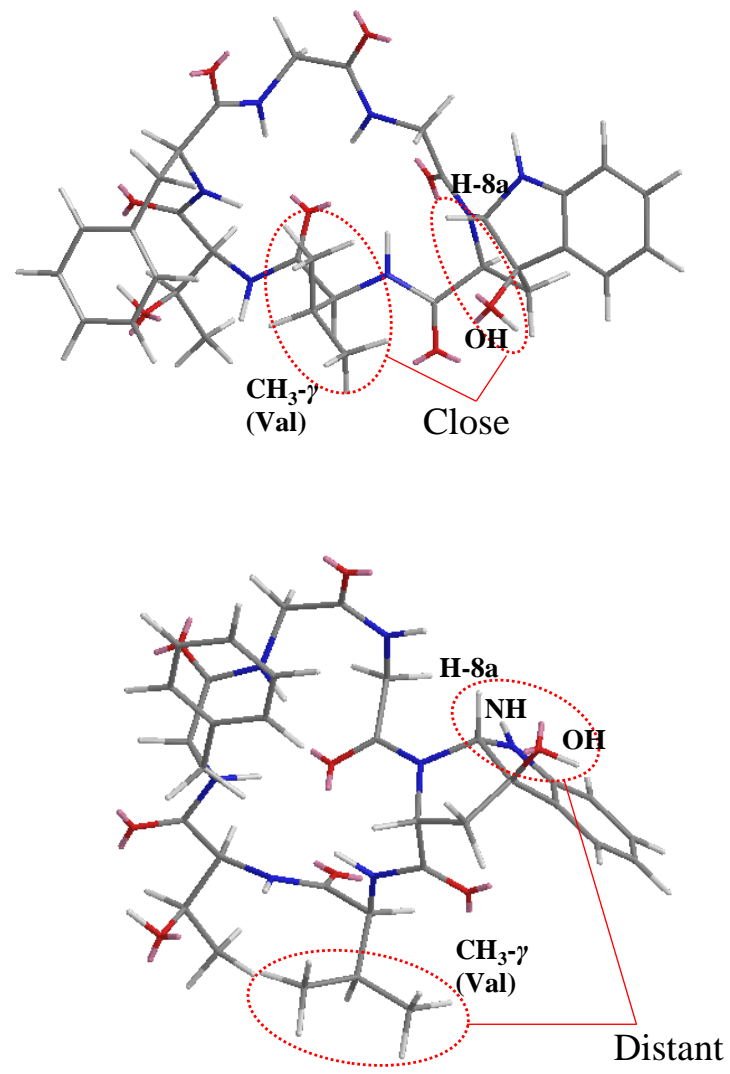

Distant

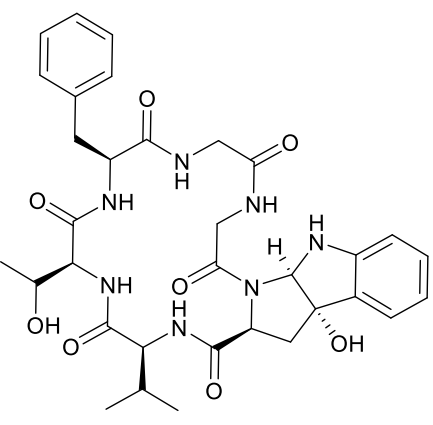

anti-cis(endo) Compound 4

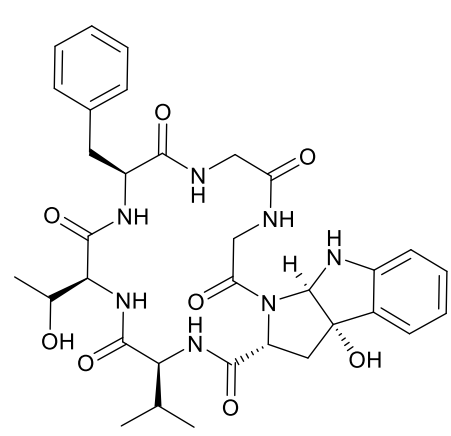

syn-cis(endo)
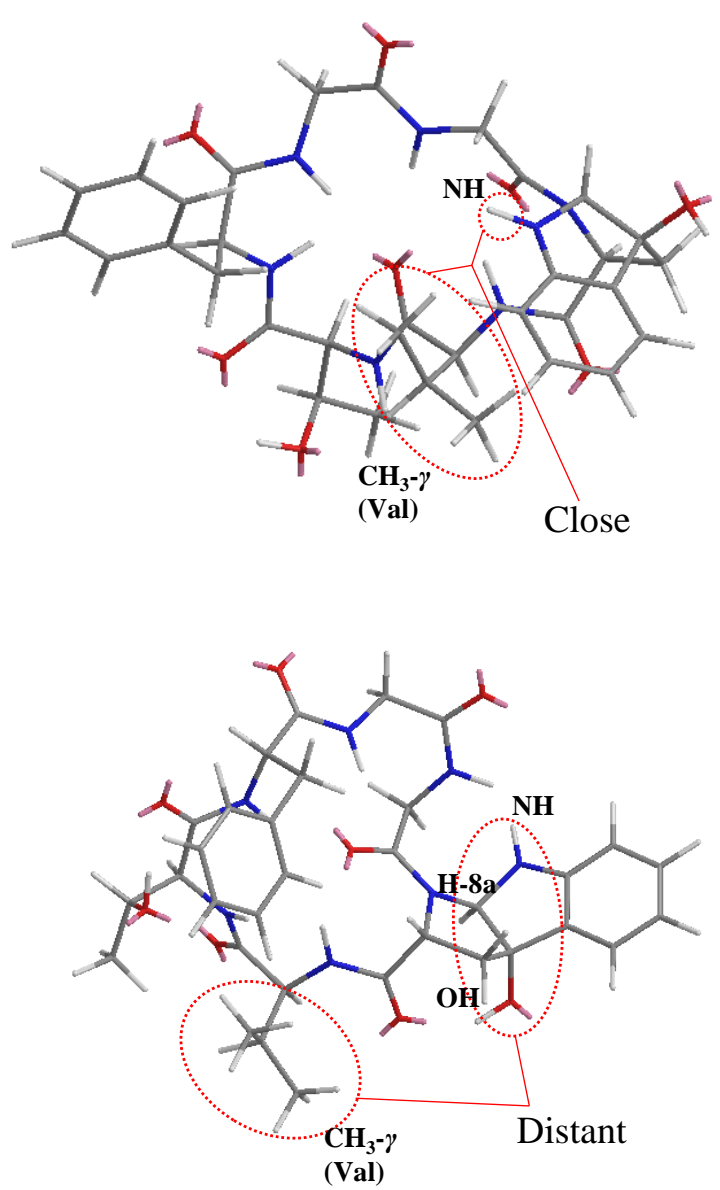

Figure S54: Distance between $\mathrm{CH}_{3}-\gamma$ (Val) and protons at the junction of HPI in 3D structures of four possible configurations of HPI. 

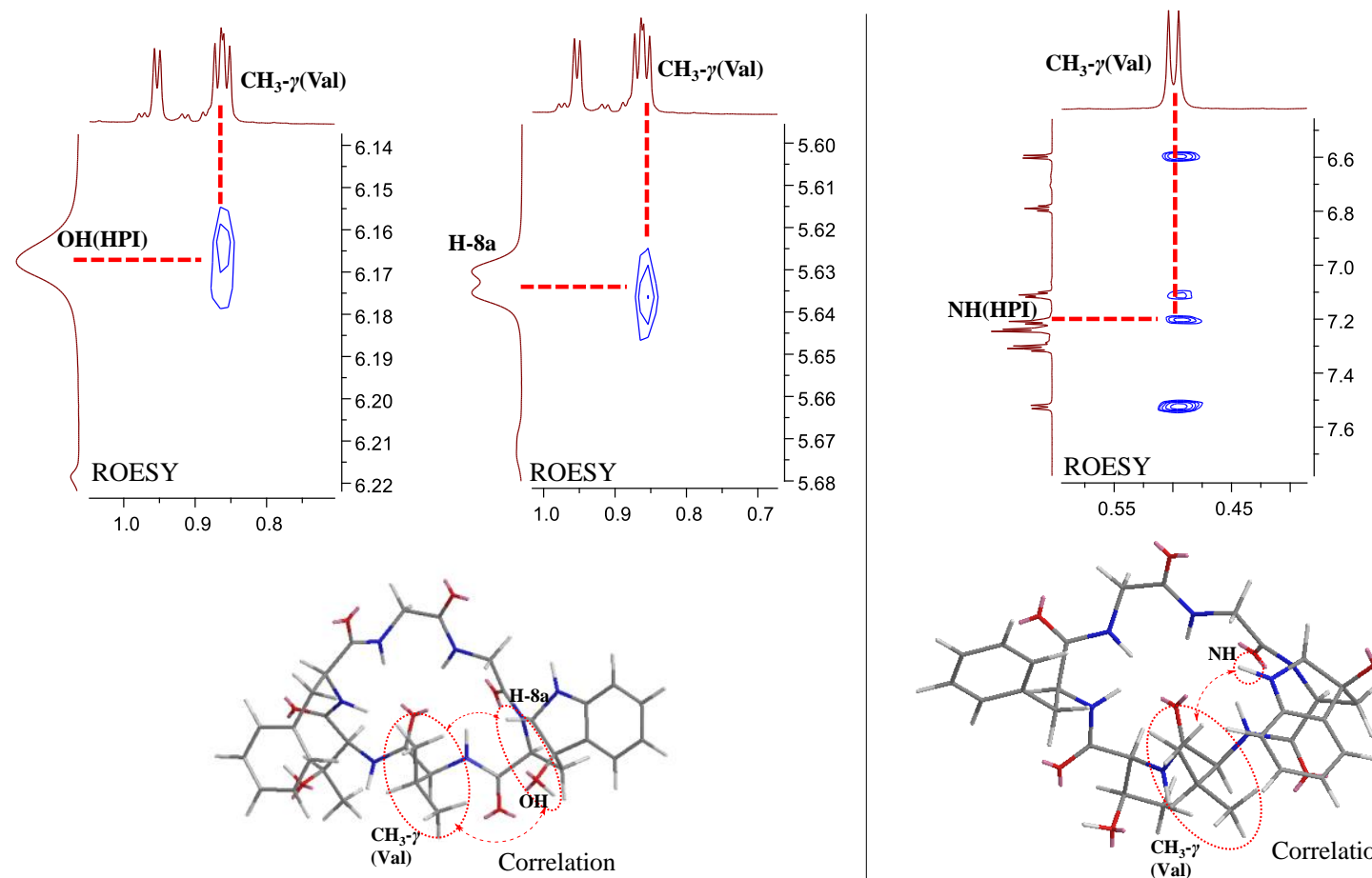

syn-cis(exo)

Compound 3

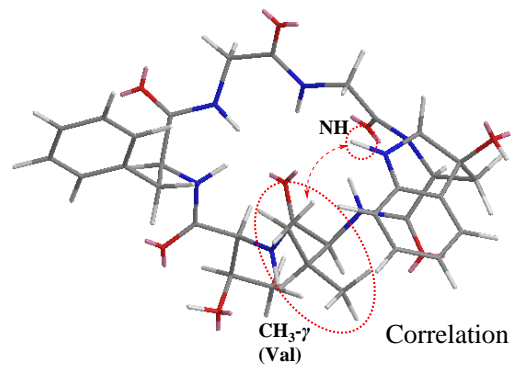

anti-cis(endo)

Compound 4

Figure S55: Expanded ROESY spectrum for correlations between $\mathrm{CH}_{3}-\gamma(\mathrm{Val})$ and protons at the junction of HPI. 

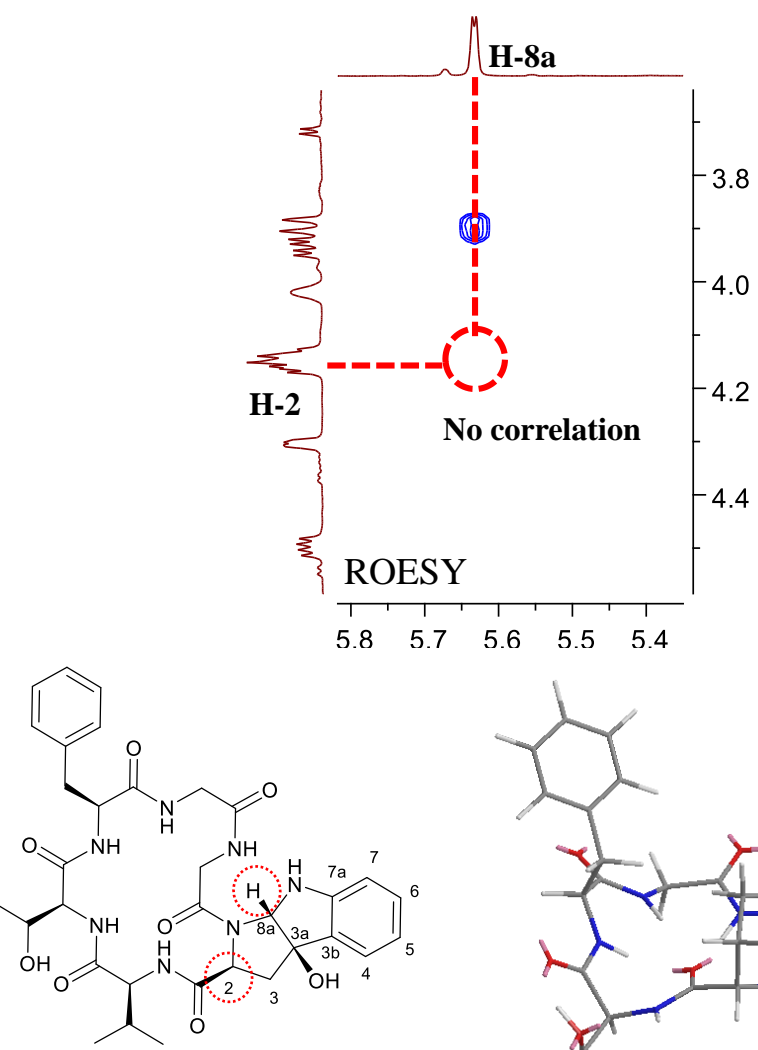

syn-cis(exo)

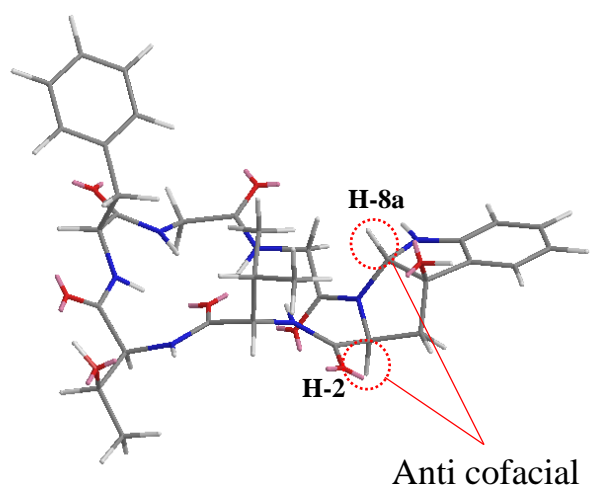

Compound $\mathbf{3}$

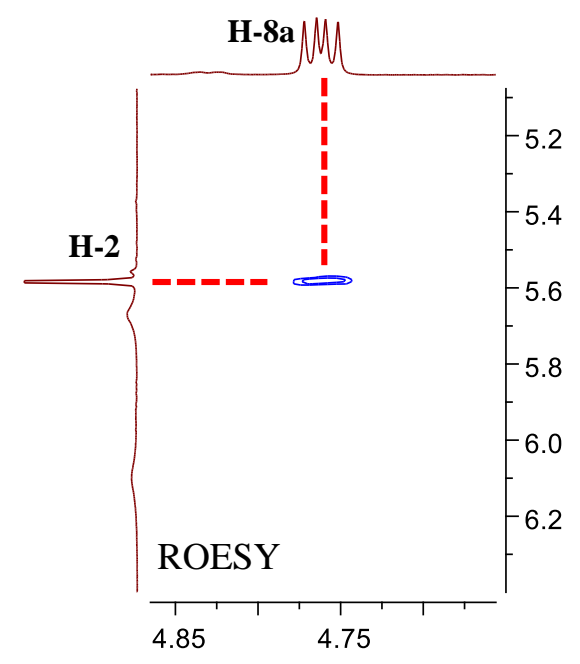

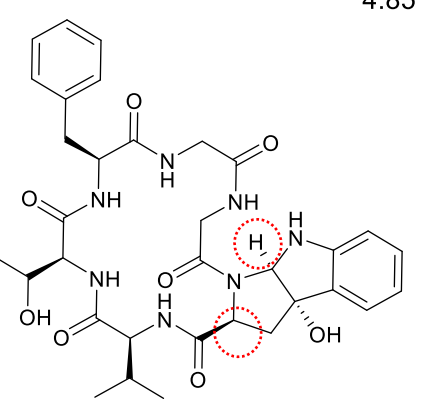

anti-cis(endo)

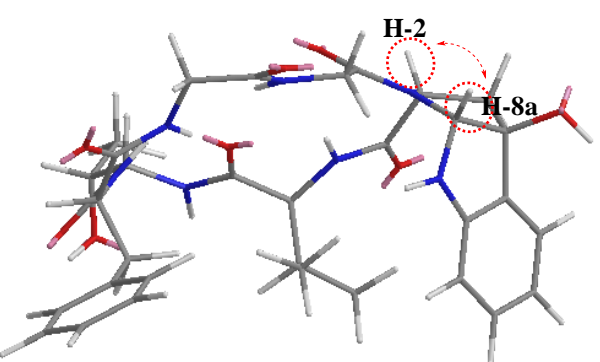

Cofacial $\rightarrow$ Correlation
Compound 4

Figure S56: Expanded ROESY spectrum for correlations between H-8a and H-2 of HPI. 
Table S3: Comparison of chemical shifts of $\mathrm{H}-2$ and $\mathrm{H}-8$ a to values in a reference.

\begin{tabular}{ccccc}
\hline & Compound & H-2 (ppm) & H-8a $(\mathrm{ppm})$ & Solvent \\
\hline \multirow{2}{*}{ Ref) } & $12 \mathrm{~b}^{*}$ & 3.86 & 5.40 & $\mathrm{D}_{2} \mathrm{O}$ \\
& $11 \mathrm{~b}^{*}$ & 4.34 & 5.31 & $\mathrm{D}_{2} \mathrm{O}$ \\
& & & & \\
M. pteleifolia & compound $\mathbf{3}$ & 4.16 & 5.63 & DMSO- $d_{6}$ \\
& compound $\mathbf{4}$ & 4.76 & 5.58 & DMSO- $d_{6}$ \\
& compound $\mathbf{5}$ & 4.18 & 5.65 & DMSO- $d_{6}$ \\
\hline
\end{tabular}

* Nakagawa et al., 1981<smiles>O=C(O)C1CC2(O)c3ccccc3N[C@H]2N1</smiles>

syn-cis(exo)

$12 \mathrm{~b} *$<smiles>CC(C)C(Cc1ccccc1)NC(=O)C(NC(=O)C(NC(=O)C(Cc1ccccc1)NC(=O)CNC(=O)NCC(=O)N1[C@H](C)C[C@@]2(O)c3ccccc3N[C@]12C)C(C)O)C(C)C</smiles>

syn-cis(exo)

Compound 3

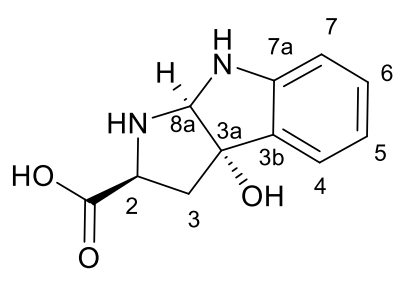

anti-cis(endo)

$11 \mathrm{~b} *$<smiles>CC(O)C(=O)NC(C(=O)NC(Cc1ccccc1)C(=O)NCC(=O)NCC(=O)N1C(C(=O)NC(C(=O)NC(Cc2ccccc2)C(=O)O)C(C)C)C[C@@]2(O)c3ccccc3NC12)C(C)C</smiles>

anti-cis(endo)

Compound 4

Figure S57: Structures of two diastereomers of HPI. 

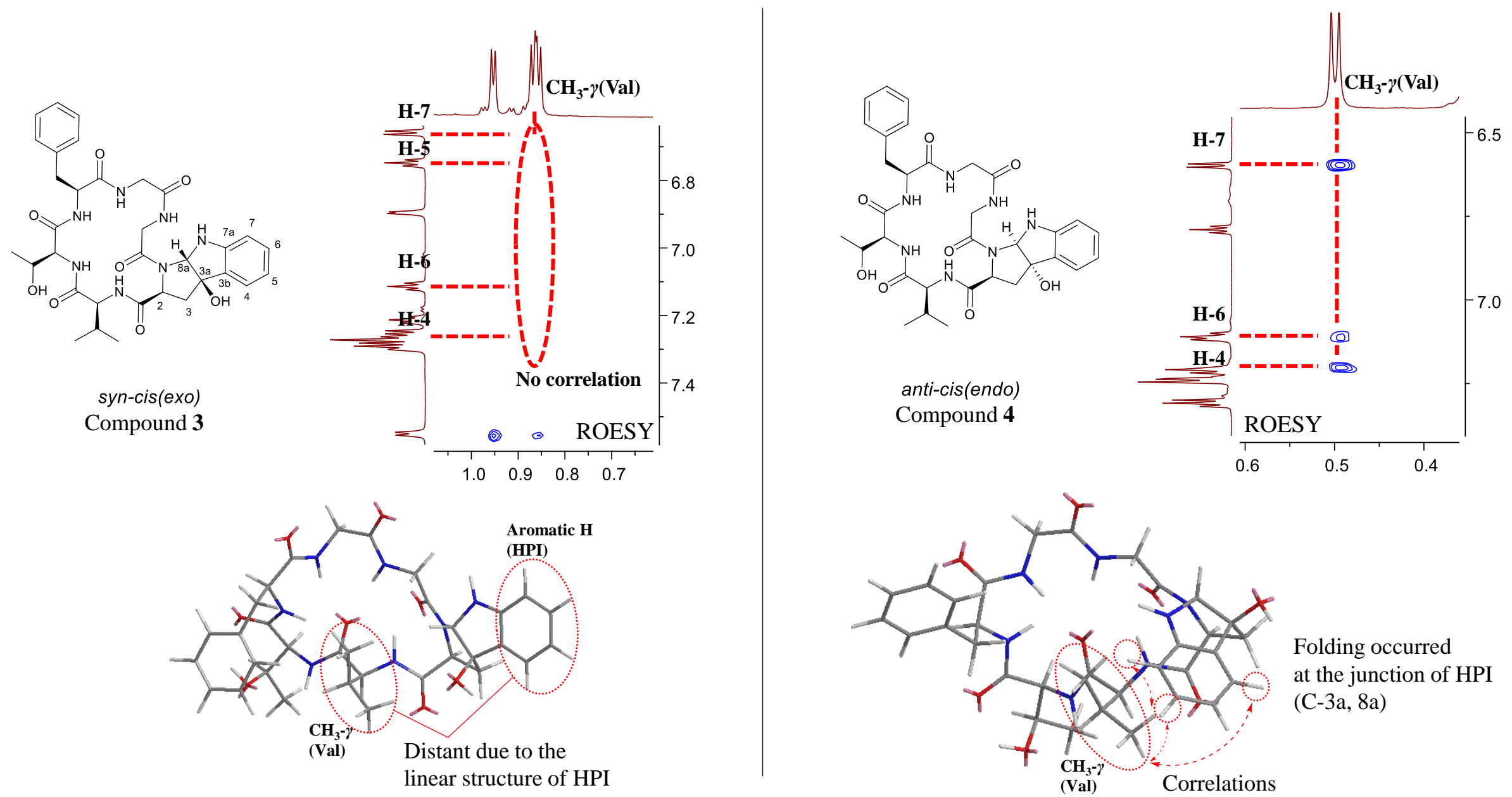

Figure S58: Expanded ROESY spectrum for correlations between $\mathrm{CH}_{3}-\gamma(\mathrm{Val})$ and aromatic protons of HPI. 
181218_ELEAnew_neg

181218_ELEAnew_neg

${ }^{100}$ Cpd 5 substituted by Try

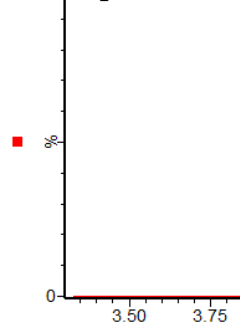

181218_ELEAnew_neg

$\left.{ }^{100}\right]$ Cpd 3 substituted by Try

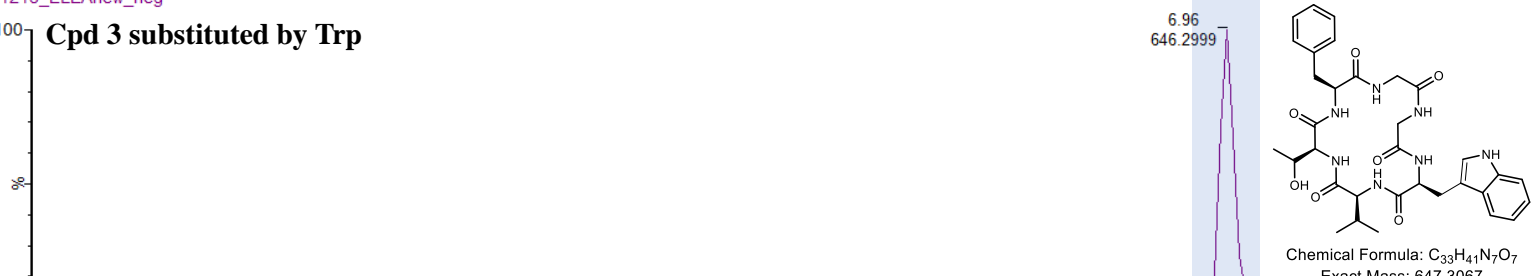

Exact Mass: 647.3067
1: TOF MS ES-

674
$1.30 \mathrm{e} 5$

181218 ELEA new 3.50

${ }_{100}^{181218 \text { ELEAnew_neg }}$ EtOAc fraction

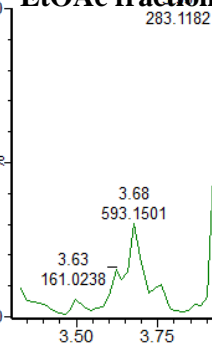

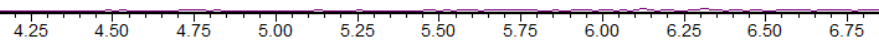

7.00
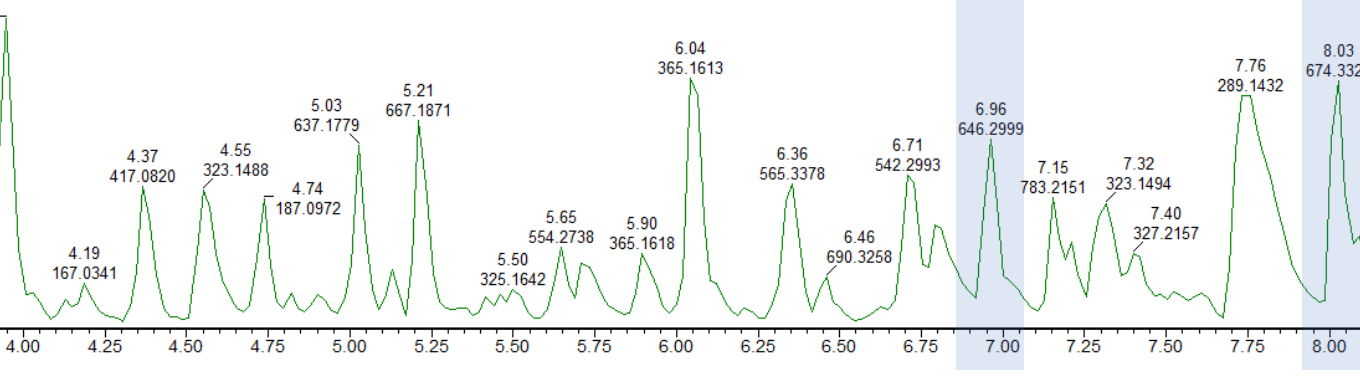
Exact Mass: 675.3380

Figure S59: Chromatograms of EtOAc fraction of M. pteleifolia and extracted ion chromatograms of compounds 3 and 5 containing Tip instead of HPI. 